\title{
Domain wall nanoelectronics
}

\author{
G. Catalan \\ Institut Catala de Recerca i Estudis Avançats (ICREA), 08193, Barcelona, Spain \\ Centre d'Investigacions en Nanociencia i Nanotecnologia (CIN2), CSIC-ICN, \\ Bellaterra 08193, Barcelona, Spain
}

J. Seidel

Materials Sciences Division, Lawrence Berkeley National Laboratory, Berkeley, California 94720, USA

Department of Physics, University of California at Berkeley, Berkeley, California 94720, USA

School of Materials Science and Engineering, University of New South Wales, Sydney NSW 2052, Australia

\section{R. Ramesh}

Materials Sciences Division, Lawrence Berkeley National Laboratory, Berkeley, California 94720, USA

Department of Physics, University of California at Berkeley, Berkeley, California 94720, USA

Department of Materials Science and Engineering, University of California at Berkeley,

Berkeley, California 94720, USA

\section{J. F. Scott}

Department of Physics, Cavendish Laboratory, University of Cambridge, Cambridge CB3 OHE, United Kingdom

(published 3 February 2012)

\begin{abstract}
Domains in ferroelectrics were considered to be well understood by the middle of the last century: They were generally rectilinear, and their walls were Ising-like. Their simplicity stood in stark contrast to the more complex Bloch walls or Néel walls in magnets. Only within the past decade and with the introduction of atomic-resolution studies via transmission electron microscopy, electron holography, and atomic force microscopy with polarization sensitivity has their real complexity been revealed. Additional phenomena appear in recent studies, especially of magnetoelectric materials, where functional properties inside domain walls are being directly measured. In this paper these studies are reviewed, focusing attention on ferroelectrics and multiferroics but making comparisons where possible with magnetic domains and domain walls. An important part of this review will concern device applications, with the spotlight on a new paradigm of ferroic devices where the domain walls, rather than the domains, are the active element. Here magnetic wall microelectronics is already in full swing, owing largely to the work of Cowburn and of Parkin and their colleagues. These devices exploit the high domain wall mobilities in magnets and their resulting high velocities, which can be supersonic, as shown by Kreines' and co-workers 30 years ago. By comparison, nanoelectronic devices employing ferroelectric domain walls often have slower domain wall speeds, but may exploit their smaller size as well as their different functional properties. These include domain wall conductivity (metallic or even superconducting in bulk insulating or semiconducting oxides) and the fact that domain walls can be ferromagnetic while the surrounding domains are not.
\end{abstract}

DOI: $10.1103 /$ RevModPhys.84.119

PACS numbers: 77.80.Fm, 68.37.Ps, 77.80.Dj, 73.61.Le

\section{CONTENTS}

I. Introduction

II. Domains

A. Boundary conditions and the formation of domains

B. Kittel's law

C. Wall thickness and universality of Kittel's law

D. Domains in nonplanar structures

E. The limits of the square root law: Surface effects, critical thickness, and domains in superlattices
F. Beyond stripes: Vertices, vortices, quadrupoles, and other topological defects

G. Nanodomains in bulk 128

H. Why does domain size matter? 130

III. Domain Walls

A. Permissible domain walls: Symmetry and compatibility conditions

B. Domain wall thickness and domain wall profile 131

C. Domain wall chirality

D. Domain wall roughness and fractal dimensions 
E. Multiferroic walls and phase transitions inside domain walls

136

F. Domain wall conductivity

IV. Experimental Methods for the Investigation of Domain Walls
A. High-resolution electron microscopy and spectroscopy
B. Scanning probe microscopy
C. X-ray diffraction and imaging
D. Optical characterization

V. Applications of Domains and Domain Walls

A. Periodically poled ferroelectrics

1. Application of Kittel's law to electro-optic domain engineering

2. Manipulation of wall thickness

B. Domains and electro-optic response of $\mathrm{LiNbO}_{3}$

C. Photovoltaic effects at domain walls

D. Switching of domains

E. Domain wall motion: The advantage of magnetic domain wall devices

F. Emergent aspects of domain wall research

1. Conduction properties, charge, and

electronic structure

2. Domain wall interaction with defects

3. Magnetism and magnetoelectric properties of multiferroic domain walls

VI. Future Directions

\section{INTRODUCTION}

Ferroic materials (ferroelectrics, ferromagnets, ferroelastics) are defined by having an order parameter that can point in two or more directions (polarities), and be switched between them by application of an external field. The different polarities are energetically equivalent, so in principle they all have the same probability of appearing as the sample is cooled down from the paraphase. Thus, zero-field-cooled ferroics can, and often do, spontaneously divide into small regions of different polarity. Such regions are called "domains," and the boundaries between adjacent domains are called "domain walls" or "domain boundaries." The ordered phase has a lower symmetry compared to the parent phase, but the domains (and consequently domain walls) capture the symmetry of both the ferroic phase and the paraphase. For example, a cubic phase undergoing a phase transition into a rhombohedral ferroelectric phase will exhibit polar order along the eight equivalent 111-type crystallographic directions, and domain walls in such a system separate regions with diagonal long axes that are $71^{\circ}, 109^{\circ}$, and $180^{\circ}$ apart. We begin our description with a general discussion of the causes of domain formation, approaches to understanding the energetics of domain size, factors that influence the domain wall energy and thickness, and a taxonomy of the different domain topologies (stripes, vertices, vortices, etc.). As the article unfolds, we endeavor to highlight the commonalities and critical differences between various types of ferroic systems.

Although metastable domain configurations or defectinduced domains can and often do occur in bulk samples, an ideal (defect-free) infinite crystal of the ferroic phase is expected to be most stable in a single-domain state (Landau and Lifshitz). Domain formation can thus be regarded in some respect as a finite size effect, driven by the need to minimize surface energy. Self-induced demagnetization or depolarization fields cannot be perfectly screened and always exist when the magnetization or polarization has a component perpendicular to the surface. Likewise, residual stresses due to epitaxy, surface tension, shape anisotropy, or structural defects induce twinning in all ferroelastics and most ferroelectrics. In general, then, the need to minimize the energy associated with the surface fields overcomes the barrier for the formation of domain walls and hence domains appear. Against this background, there are two observations and a corollary that constitutes the core of this review:

(1) The surface-to-volume ratio grows with decreasing size; consequently, small devices such as thin films, which are the basis of modern electronics, can have small domains and a high volume concentration of domain walls.

(2) Domain walls have different symmetry, and hence different properties, from those of the domains they separate.

The corollary is that the overall behavior of the films may be influenced, or even dominated, by the properties of the

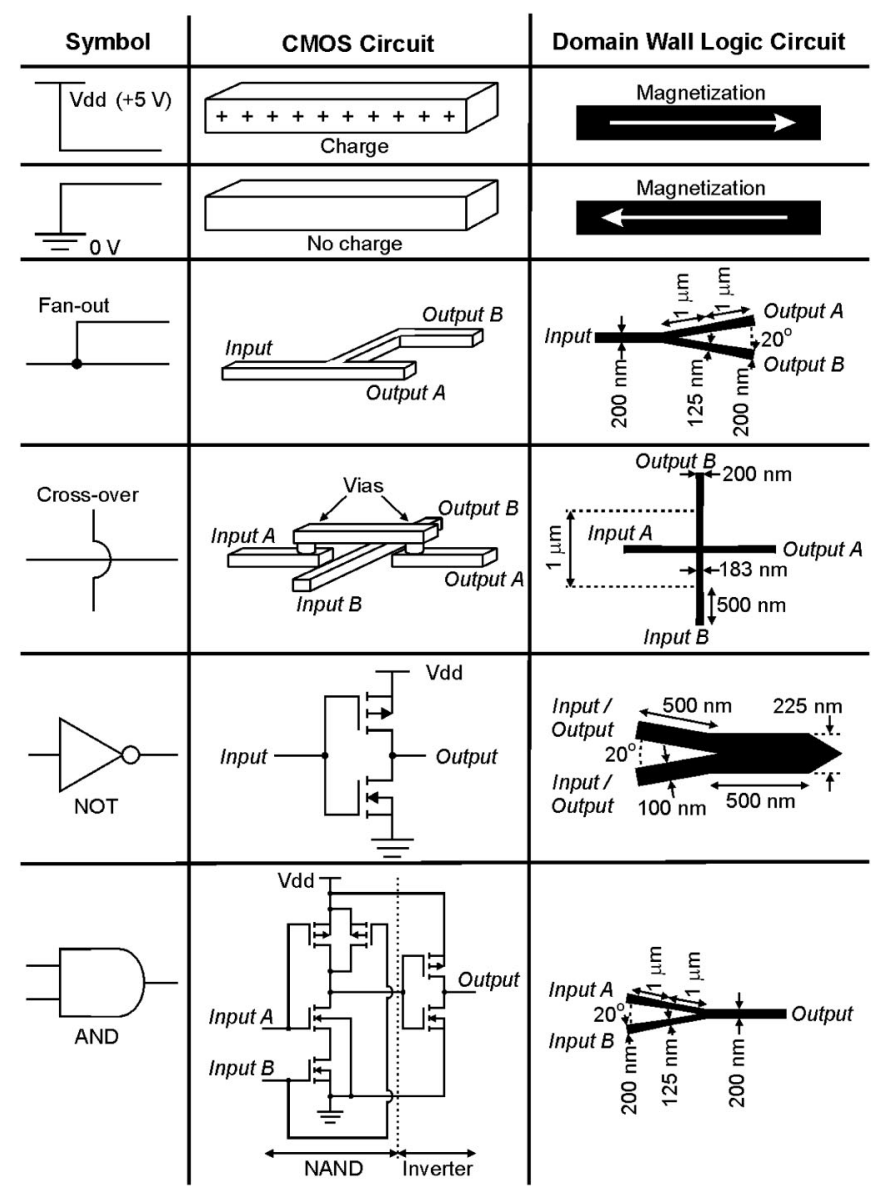

FIG. 1. Schematic of logic circuits where the active element is not charge, as in current complementary metal oxide semiconductor (CMOS) technology, but domain wall magnetism. From Allwood et al., 2005. 
walls, which are different from those of the bulk material. Moreover, not only do domain walls have their own properties but, in contrast to other types of interface, they are mobile. One can therefore envisage new technologies where mobile domain walls are the "active ingredient" of the device, as highlighted by Salje (2010). A prominent example of this idea is the magnetic "racetrack memory" where the domain walls are pushed by a current and read by a magnetic head (Parkin, Hayashi, and Thomas, 2008); in fact, the entire logic of an electronic circuit can be reproduced using magnetic domain walls (Allwood et al., 2005) (see Fig. 1).

Herbert Kroemer, Physics Nobel Laureate in 2000 for his work on semiconductor heterostructures, is often quoted for his dictum "the interface is the device." He was, of course, referring to the interfaces between different semiconductor layers. His ideas were later extrapolated, successfully, to oxide materials, where the variety of new interface properties seems to be virtually inexhaustible (Mannhart and Schlom, 2010; Zubko et al., 2011). However, this review is about a different type of interface: not between different materials, but between different domains in the same material. Paraphrasing Kroemer, then, our aim is to show that "the wall is the device."

\section{DOMAINS}

\section{A. Boundary conditions and the formation of domains}

The presence and size of domains (and therefore the concentration of domain walls) in any ferroic depends on its boundary conditions. Consider, for example, ferroelectrics. The surfaces of a ferroelectric material perpendicular to its polar direction have a charge density equal to the dipolar moment per unit volume. This charge generates an electric field of sign opposite to the polarization and magnitude $E=P / \varepsilon$ (where $\varepsilon$ is the dielectric constant). For a typical ferroelectric $\left(P=10 \mu \mathrm{C} / \mathrm{cm}^{2}, \varepsilon_{r}=100-1000\right)$, this depolarization field is c.a. $10-100 \mathrm{kV} / \mathrm{cm}$, which is about an order of magnitude larger than typical coercive fields. So, if nothing compensates the surface charge, the depolarization will in fact cancel the ferroelectricity. Charge supplied by electrodes can partly screen this depolarization field and, although the screening is never perfect (Batra and Silverman, 1972; Dawber, Jung, and Scott, 2003; Dawber et al., 2003; Stengel and Spaldin, 2006), good electrodes can stabilize ferroelectricity down to films just a few unit cells thick (Junquera and Ghosez, 2003). But a material can also reduce the self-field by dividing the polar ground state into smaller regions (domains) with alternating polarity, so that the average polarization (or spin, or stress, depending on the type of ferroic material considered) is zero. Although this does not completely get rid of the depolarization (locally, each individual domain still has a small stray field), the mechanism is effective enough to allow ferroelectricity to survive down to films of only a few unit cells thick (Streiffer et al., 2002; Fong et al., 2004). The same samples (e.g., epitaxial $\mathrm{PbTiO}_{3}$ on $\mathrm{SrTiO}_{3}$ substrates) can in fact show either extremely small (a few angstroms) domains or an infinitely large monodomain configuration just by changing the boundary condition (Fong et al., 2006), i.e., by allowing

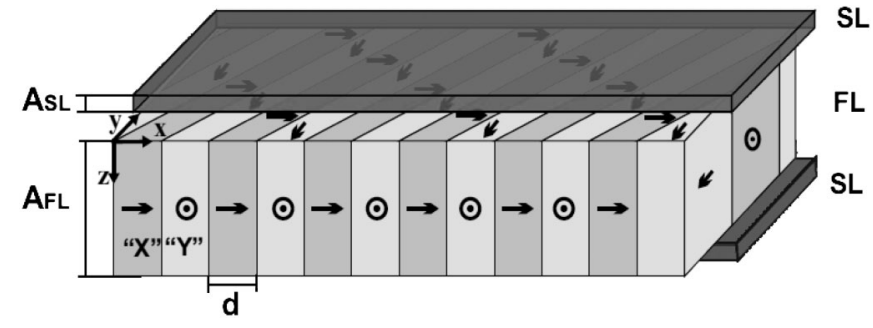

FIG. 2. Surface "dead" layers that do not undergo the ferroic transition can cause the appearance of ferroelastic twins in otherwise stress-free films. Dead layers also exist in other ferroics such as ferroelectrics and ferromagnets. From Luk'yanchuk et al., 2009.

free charges to screen the electric field so that the formation of domains is no longer necessary (and it is noteworthy that such effective charge screening can be achieved just by adsorbates from the atmosphere).

An important boundary condition is the presence or otherwise of interfacial "dead layers" that do not undergo the ferroic transition. Dead layers have been discussed in the context of ferroelectrics, where they are often proposed as explanations for the worsening of the dielectric constant of thin films, although the exact nature, thickness, and even location of the dead layer, which might be inside the electrode, is still a subject of debate (Sinnamon, Bowman, and Gregg, 2001; Stengel and Spaldin, 2006; Chang et al., 2009). In ferroelectrics, dead layers prevent screening causing domains to appear (Bjorkstam and Oettel, 1967; Kopal et al., 1999; Bratkovsky and Levanyuk, 2000). More recently, Luk'yanchuk et al. (2009) proposed that an analogous phenomenon may take place in ferroelastics, so that "ferroelastic dead layers" can cause the formation of twins (Fig. 2). Surfaces have broken symmetries and are thus intrinsically uncompensated, so interfacial layers are likely to be a general property of all ferroics, including, of course, multiferroics (Marti et al., 2011).

\section{B. Kittel's law}

For the sake of simplicity, most of this discussion will assume ideal open boundary conditions and no screening of surface fields. The geometry of the simplest domain morphology, namely, stripe domains, is depicted in Fig. 3. Although a

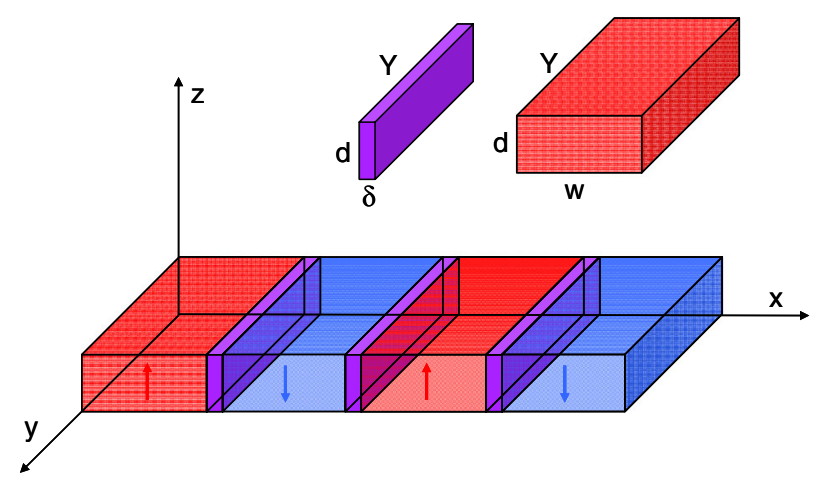

FIG. 3 (color online). Schematic of the geometry of $180^{\circ}$ stripe domains in a ferroelectric or a ferromagnet with out-of-plane polarity. 
stripe domain is by no means the only possible domain structure, it is the most common (Edlund and Jacobi, 2010) and conceptually the simplest. It also captures the physics of domains that is common to all types of ferroic materials. For more specialized analyses, the reader is referred to monographs about domains in different ferroics: ferromagnets (Hubert and Schafer, 1998), ferroelectrics (Tagantsev, Cross, and Fousek, 2010), and ferroelastics or martensites (Khachaturyan, 1983).

Domain size is determined by the competition between the energy of the domains (itself dependent on the boundary conditions, as emphasized above) and the energy of the domain walls. The energy density of the domains is proportional to the domain size: $E=U w$, where $U$ is the volume energy density of the domain and $w$ is the domain width. Smaller domains therefore have smaller depolarization, demagnetization, and elastic energies. But the energy gained by reducing domain size is balanced by the fact that this requires increasing the number of domain walls, which are themselves energetically costly.

The energy cost of the domain walls increases linearly with the number of domain walls in the sample, and therefore it is inversely proportional to the domain size $(n=1 / w)$. Meanwhile, the energy of each domain wall is proportional to its area and, thus, to its vertical dimension. If an individual domain wall stopped halfway through the sample, the polarity beyond the end point of the wall would be undefined, so, topologically, a domain wall cannot do this; it must either end in another wall (as it does for needle domains) or else cross the entire thickness of the sample. For walls that cross the sample, the energy is proportional to the sample thickness. Thus, the walls' energy density per unit area of thin film is $E=\sigma d / w$, where $\sigma$ is the energy density per unit area of the wall. Adding up the energy costs of domains and domain walls, and minimizing the total with respect to the domain size, leads to the famous square root dependence:

$$
w=\sqrt{\frac{\sigma}{U} d} .
$$

Landau and Lifshitz (1935) and Kittel (1946) proposed this pleasingly simple model within the context of ferromagnetism, where the domain energy was provided by the demagnetization field (assuming spins pointing out of plane). It is nevertheless interesting to notice that Kittel's classic article predicted that pure stripes were in fact energetically unfavorable compared to other magnetic domain configurations (see Fig. 4); this is because his calculations were performed for magnets with relatively small magnetic anisotropy. Where the anisotropy is large, as in cobalt, stripes are favored, and this is also the case for uniaxial ferroelectrics or for perovskite ferroelectrics under in-plane compressive strain (which strongly favors out-of-plane polarization). Closure domains are common in ferromagnets (where anisotropy is intrinsically smaller than in ferroelectrics), but the width of the "closure stripes" also scales as the square root of the thickness (Kittel, 1946). We return again to the subject of closure domains toward the end of
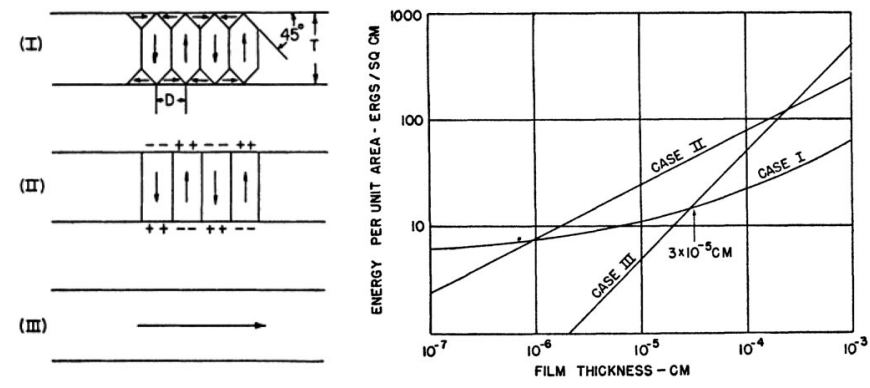

FIG. 4. Kittel's classic study of the minimum energy of different domain configurations: I are "closure stripes" with no demagnetization; II are conventional stripes; and III is a monodomain with the polar direction in plane. Note that in the early calculations for magnetic domains, the conventional stripes were not stable at any finite thickness, due to the small anisotropy assumed. From Kittel, 1946.

this section, as it has become a hot topic in the area of ferroelectrics and multiferroics.

Kittel's law was extended by Mitsui and Furuichi (1953) for ferroelectrics with $180^{\circ}$ domain walls, by Roitburd (1976) for ferroelastic thin films under epitaxial strain, by Pompe et al. (1993) and Pertsev and Zembilgotov (1995) for epitaxial films that are simultaneously ferroelectric and ferroelastic, and, more recently, by Daraktchiev, Catalan, and Scott (2008) for magnetoelectric multiferroics. The square root dependence of stripe domain width on film thickness is therefore a general property of all ferroics, and it also holds for other periodic domain patterns (Kinase and Takahashi, 1957; Craik and Cooper, 1970; Thiele, 1970).

\section{Wall thickness and universality of Kittel's law}

The exact mathematical treatment of the "perfect stripes" model assumes that the domain walls have zero or at least negligible thickness compared to the width of the domains. In reality, however, domain walls do have a finite thickness $\delta$, which depends on material constants (Zhirnov, 1959). Scott (2006) observed that for each given material one could rewrite the square root dependence as

$$
\frac{w^{2}}{\delta d}=G
$$

where $G$ is an adimensional parameter. This equation is also useful in that it can be used in reverse in order to estimate the domain wall thickness of any ferroic with well-defined boundary conditions (Catalan et al., 2007a). Indirect versions of it have been calculated for the specific case of ferroelectrics (Lines and Glass, 2004; De Guerville et al., 2005), but in fact Eq. (2) is independent of the type of ferroic and allows comparisons between different material classes. Schilling et al. (2006a) did such a comparison and showed explicitly that, while all ferroics scaled with a square root law, ferromagnetic domains were wider than ferroelectric domains. Meanwhile, the walls of ferromagnets are also much thicker than those of ferroelectrics (Zhirnov, 1959), so that when the square of the domain size is divided by the wall thickness as per Eq. (2), all ferroics look the same (see Fig. 5), meaning 


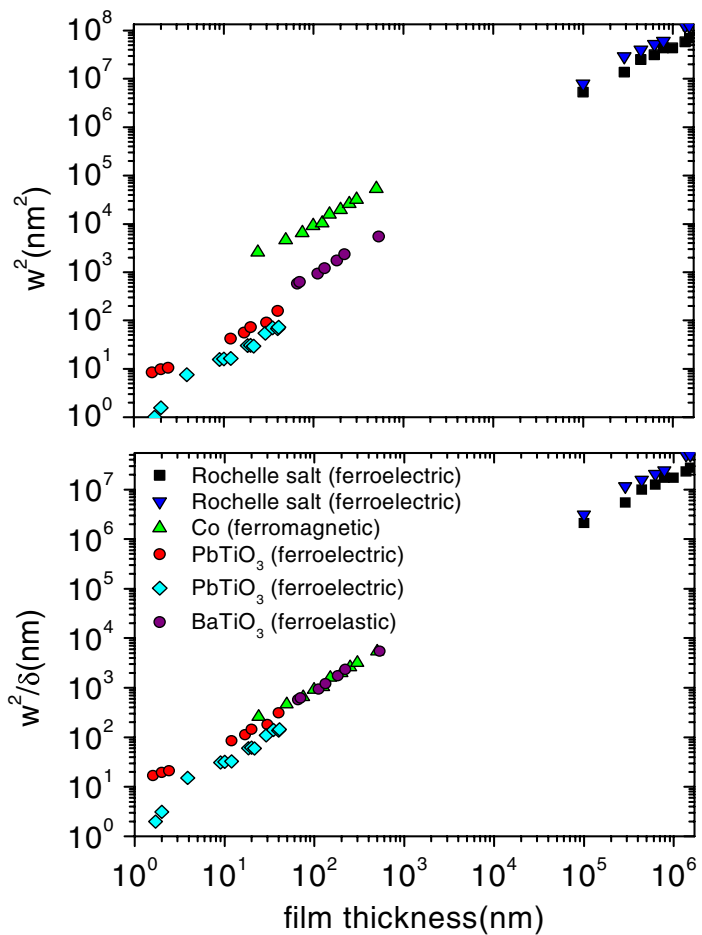

FIG. 5 (color online). Comparisons between stripe domains of different ferroic materials show (i) that all of them scale with the same square root dependence of domain width on film thickness; (ii) that Kittel's law holds true for ferroelectrics down to small thickness; (iii) that when the square of the domain size is normalized by the domain wall thickness, the different ferroics fall on pretty much the same master curve. Adapted from Catalan et al., 2009.

that $G$ is the same for all the different ferroics. The value of $G$ has been calculated (De Guerville et al., 2005; Catalan et al., 2007a, 2009) ${ }^{1}$ as

$$
G=1.765 \sqrt{\frac{\chi_{x}}{\chi_{z}}}
$$

where $G$ depends on the anisotropy between in-plane $\left(\chi_{x}\right)$ and out-of-plane $\left(\chi_{z}\right)$ susceptibilities, but in practice the dependence on material properties is weak because they are inside a square root.

Equation (2) is useful in several ways. First, it allows one to estimate domain wall thicknesses just by measuring domain sizes, and it is easier to measure wide domains than it is to measure narrow domain walls. As we discuss in the following sections, domain wall thicknesses have traditionally been difficult to determine precisely due to their narrowness (see Sec. III.B). Second, Eq. (2) is also a useful guide as

\footnotetext{
${ }^{1}$ We note that different values have been given for the exact numerical coefficient. The discrepancies are typically factors of 2 and are due to the different conventions regarding whether $\delta$ is the domain wall thickness or the correlation length, and whether $w$ is the domain width or the domain period. It is therefore important to carefully define the parameters: Here $\delta$ is twice the correlation length (which is a good approximation to the wall thickness), whereas $w$ is the domain size (half the domain period).
}

to what the optimum crystal thickness should be in order to stabilize a given domain period, and this may be useful, for example, in the fabrication of periodically poled ferroelectrics for enhancement of the second-harmonic generation. Specific examples of this are discussed in detail in Sec. V of this review.

Although Eq. (2) may appear slightly "miraculous" in that it links in a simple and useful way some quantities that are not at first sight related, closer inspection removes the mystery. A direct comparison between Eqs. (1) and (2) shows that at heart, the domain wall renormalization of Kittel's law is a consequence of the fact that the domain wall surface energy density $\sigma$ is of the order of the volume energy density $U$ integrated over the thickness of the domain wall $\delta$, i.e., $\sigma \sim U \delta$, which one could have guessed just from a dimensional analysis. We emphasize also that these equations are derived assuming open boundary conditions and are not valid when the surface fields are screened.

\section{Domains in nonplanar structures}

Kittel's simple arguments can be adapted to describe more complex geometries. For instance, one can extend them to calculate domain size in nonplanar structures such as nanowires and nanocrystals or nanodots. The interest in these three-dimensional structures stems originally from the fact that they allow the reduction of the on-chip footprint of memory devices. The size of the domains in simple threedimensional shapes such as, say, a parallelepiped (cuboid) can be readily rationalized by adding up the energy of the domain walls plus the surface energy of the six faces of the parallelepiped with lateral dimensions $d_{x}, d_{y}$, and $d_{z}$. Minimizing this with respect to domain width $w$ leads to (Catalan et al., 2007b)

$$
w^{2}=\frac{\sqrt{2}}{2} \frac{\sigma}{\left(U_{x} / d_{x}\right)+\left(U_{y} / d_{y}\right)+\left(U_{z} / d_{z}\right)},
$$

where $\sigma$ is the energy per unit area of the domain walls, and $U_{x}, U_{y}$, and $U_{z}$ are the contributions to the volume energy density coming from the $x, y$, and $z$ facets of the domains. Equation (4) becomes the standard Kittel law when two of the dimensions are infinite (thin-film approximation). It can also be seen that domains become progressively smaller as the sample goes from thin film (one finite dimension) to column (two finite dimensions) to nanocrystal (three finite dimensions) (Schilling et al., 2009).

These arguments also work for the grains of a polycrystalline sample (ceramic or nonepitaxial film), which are generally found to have small domains that scale as the square root of the grain size rather than the overall size dimensions (Arlt, 1990). Arlt also observed and rationalized the appearance of bands of correlated stripe domains, called "herringbone" domains (see Fig. 6) (Arlt and Sasko, 1980; Arlt, 1990). The concept of correlated clusters of domains was later generalized for more complex structures as "metadomains" or "bundle domains" (Ivry, Chu, and Durkan, 2010), and their local functional response was studied using piezoresponse force microscopy (PFM) (Anbusathaiah et al., 2009; 



FIG. 6. (Left) Classic herringbone twin domain structure in large grains of ferroelastic ceramics, and (right) bundles of correlated stripes in smaller grains. From Arlt, 1990.

Ivry, Chu, and Durkan, 2010). Herringbone domains appear only above a certain critical diameter, above which the domain size dependence gets modified: The stripes scale as the square root of the herringbone width, while the herringbone width scales as $r^{2 / 3}$ (where $r$ is the grain radius), so that the stripe width ends up scaling as $r^{1 / 3}$ (Arlt, 1990).

Randall and co-workers also studied in close detail the domain size dependence within ceramic grains (Cao and Randall, 1996; Randall et al., 1998) and concluded that the square root dependence is valid only within a certain range of grain sizes, with the scaling exponent being smaller than $\frac{1}{2}$ for grains larger than $10 \mu \mathrm{m}$, and bigger than $\frac{1}{2}$ for grains smaller than $1 \mu \mathrm{m}$. The same authors observed cooperative switching of domains across grain boundaries, as did Gruverman et al. (1995a, 1995b, 1996), evidence that the elastic fields associated with ferroelastic twinning are not easily screened and can therefore couple across boundaries.

Similar ideas underpin the description of domains in nanocolumns and nanowires, where domain size is found to be well described by Eq. (4) with one dimension set to infinity (Schilling et al., 2006b). An interesting twist is that the competition between domain energy and domain wall energy can be used not just to rationalize domain size, but to actually modulate the orientation of the domains just by changing the relative sample dimensions (Schilling et al., 2007) (see Fig. 7).

These are a few examples, but there is still work to be done. The geometry of domains in noncompact nanoshapes such as nanorings or nanotubes, for example, remains to be rationalized. The interest in such structures goes beyond purely

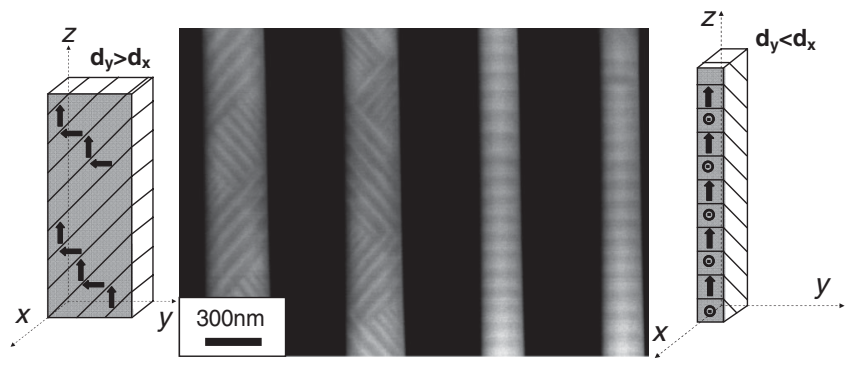

FIG. 7. Ferroelastic and ferroelectric $90^{\circ}$ domains in singlecrystal nanocolumns of $\mathrm{BaTiO}_{3}$. The domains arrange themselves so as to have the depolarizing fields only on the narrowest dimension of the column, thus minimizing the overall surface energy. Adapted from Schilling et al., 2007. academic curiosity, as ferroelectric nanotubes may have real life applications in nanoscopic fluid-delivery devices such as ink-jet printers and medical drug delivery implants. Another important question that is only beginning to be studied concerns the switching of the ferroelectric domains in such nonplanar structures: Spanier et al. (2006) showed that it was possible to switch the transverse polarization even in ultrathin nanowires ( $3 \mathrm{~nm}$ diameter), while Gregg and coworkers have shown that the longitudinal coercive field can be modified by introducing notches or antinotches along the wires (McMillen et al., 2010; McQuaid, Chang, and Gregg, 2010). The same group of authors are also pioneering research on the static and dynamic response of correlated bundles of nanodomains, showing that such metadomains can, to all intents and purposes, be treated as if they were domains in their own right (McQuaid et al., 2011).

\section{E. The limits of the square root law: Surface effects, critical thickness, and domains in superlattices}

In spite of its simplicity, the square root law holds over a remarkable range of sizes and shapes. It is natural to ask when or whether this law breaks down. For large film thickness there is no theoretical threshold beyond which the law should break down, and, experimentally, Mitsui and Furuichi (1953) observed conformance to Kittel's law in crystals of millimeter thickness. In epitaxial thin films, however, screening effects and/or defects have been reported to induce randomness and even stabilize monodomain configurations in $\mathrm{PbTiO}_{3}$ films thicker than 100 unit cells (Takahashi et al., 2008). As for the existence of a lower thickness limit, Kittel's derivation makes a number of assumptions that are size dependent. One of them is that the domain wall thickness is negligible in comparison with the domain size. Domain walls are sharp in ferroelastics and even more so in ferroelectrics (Merz, 1954; Kinase and Takahashi, 1957; Zhirnov, 1959; Padilla, Zhong, and Vanderbilt, 1996; Meyer and Vanderbilt, 2002), so that this assumption is robust all the way down to an almost atomic scale (Fong et al., 2004), but this is not the case for ferromagnets, where domain walls are thicker $(10-100 \mathrm{~nm})$. For ferromagnets, Kittel's law breaks down at film thicknesses of several tens of nanometers (Hehn et al., 1996).

A second assumption of Kittel's law is that the two surfaces of the ferroic material do not "see" each other. That is to say, the stray field lines connecting one domain to its neighbors are much denser than the field lines connecting one face of the domain to the opposite one. However, if and/ or when the size of the domains becomes comparable to the thickness of the film, the electrostatic interaction with the opposite surface starts to take over (Kopal, Bahnik, and Fousek, 1997). Takahashi et al. (2008) recently suggested that the square root law breaks down at a precise threshold value of the depolarization field. Below that critical thickness, the domain size no longer decreases but it increases again, and diverges as the film thickness approaches zero. Neglecting numerical factors of order unity and also neglecting dielectric anisotropy, the critical thickness for a ferroelectric is (Kopal, Bahnik, and Fousek, 1997; Streiffer et al., 2002) $d_{C} \approx \sigma\left(\varepsilon / P^{2}\right)$ (where $\varepsilon=\varepsilon_{0} \varepsilon_{r}$ is the average dielectric constant), while for ferroelastic twins in an epitaxial 


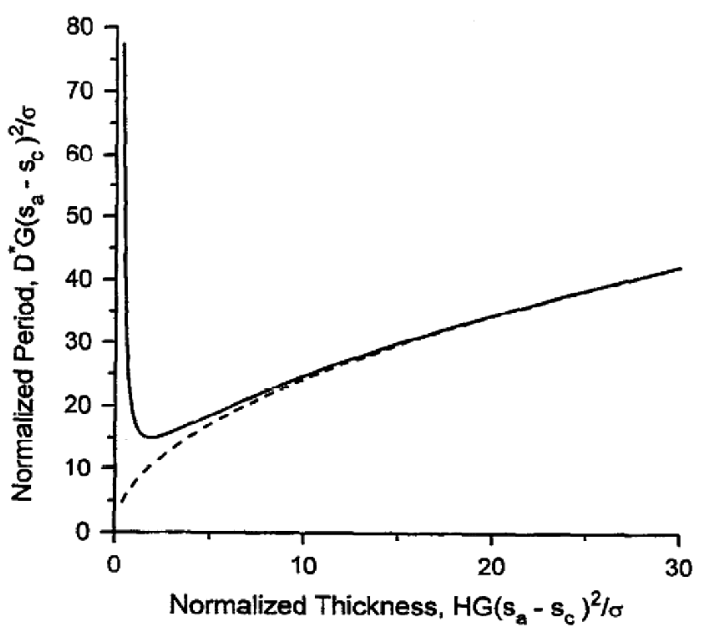

FIG. 8. Calculated domain size for $90^{\circ}$ ferroelastic domains in an epitaxial film as a function of film thickness. Below a certain critical thickness the domain size stops following the square root dependence and begins to diverge. This critical thickness is of the order of the domain wall thickness. From Pertsev and Zembilgotov, 1995.

structure it is (Pertsev and Zembilgotov, 1995) $d_{C}=$ $\left[\sigma / G\left(s_{a}-s_{c}\right)^{2}\right]$, where $G$ is the shear modulus and $s_{a}$ and $s_{c}$ are the spontaneous tetragonal strains (along $a$ and $c$ axes, respectively). The theoretical divergence from the square root law for ferroelastic twins in epitaxial films is shown in Fig. 8.

Notice that, as a rule of thumb, these critical thicknesses for domain formation are reached when the size of the domains becomes comparable to the size of the interfacial dead layers (Luk'yanchuk et al., 2009). They are typically in the 1-10 $\mathrm{nm}$ range, and therefore ferroelectric and ferroelastic domains persist even for extremely thin layers, as shown by Fong et al. (2004) for single films and by Zubko et al. (2010) in fine-period superlattices.

In the particular case of epitaxial ferroelastics there are further geometrical constraints on the domain size that are not readily captured by continuum theories. Ferroelastic twinning introduces a canting angle between the atomic planes of adjacent domains. The canting angle $\alpha$ is, for the particular case of $90^{\circ}$ twins in tetragonal materials (e.g., $\mathrm{BaTiO}_{3}$ or

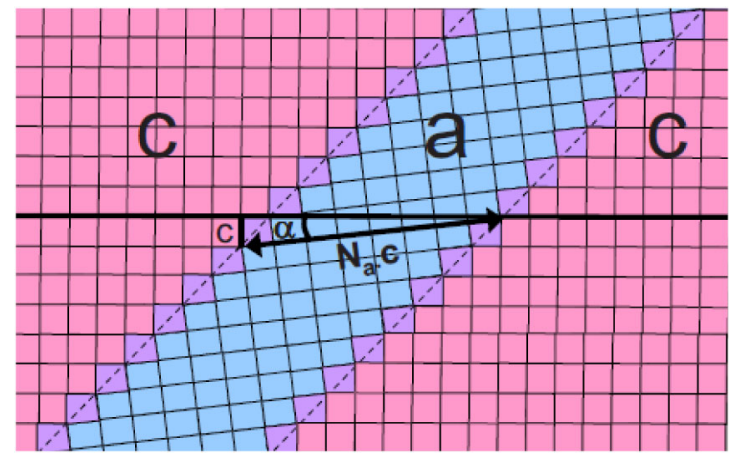

FIG. 9 (color online). Schematic of the geometrical minimum domain size in a tetragonal twin structure such that wider $c$ domains are coplanar with the substrate while the narrow $a$ domains are tilted with the inherent twinning angle $\alpha$. From Vlooswijk, Catalan, and Noheda, 2010.
$\left.\mathrm{PbTiO}_{3}\right), \alpha=90^{\circ}-2 \tan ^{-1}(a / c)$. The existence of this canting angle, combined with the tendency of the bigger domains to be coplanar with the substrate, introduces a geometrical lower limit to domain size (Vlooswijk et al., 2007): in order to ensure coplanarity between Bragg planes across the smallest domain, the minimum domain size must be $w_{a}^{\min }=$ $c / \sin (\alpha)$ (see Fig. 9). For the particular case of $\mathrm{PbTiO}_{3}$, $w_{a}^{\min }=7 \mathrm{~nm}$. This geometrical minimum domain size applies only to films that are epitaxial (Ivry, Chu, and Durkan, 2009; Vlooswijk, Catalan, and Noheda, 2010).

\section{F. Beyond stripes: Vertices, vortices, quadrupoles, and other topological defects}

A final question regarding the domain scaling issue concerns what happens to domains beyond the square root range? Other domain morphologies are possible that can be reached in extreme cases of confinement, or when the polarization is coupled to other order parameters. In the ultrathin-film regime, for example, atomistic simulations predict that the perfect $180^{\circ}$ domains of ferroelectrics should become akin to the closure configuration of ferromagnets (Kornev, Fu, and Bellaiche, 2004; Aguado-Fuente and Junquera, 2008) (see Fig. 10). It may seem preposterous to care about a domain structure that takes place only in films that are barely a few unit cells thick, but with the advent of ferroelectric superlattices these domains become accessible, as the thickness of each individual layer in the superlattice can be as thin as one single unit cell (Dawber et al., 2005; Zubko et al., 2010). In the weak-coupling regime, the ferroelectric slabs within the superlattice act as almost separate ultrathin entities (Stephanovich, Luk'yanchuk, and Karkut, 2005), so that it is quite possible that these closure stripes are achieved. It is worth noticing that the orientation of the in-plane component of the polarization is such that, if the domain walls were pushed toward each other, there would be a head-to-head collision of polarizations; the electrostatic repulsion between these in-plane components might explain why it seems to be almost impossible to eliminate the domain walls in ferroelectric superlattices (Zubko et al., 2010).

On a related note, while the $180^{\circ}$ domain walls of ferroelectrics have traditionally been considered nonchiral (i.e., the polarization just decreases, goes through zero, and increases again, but does not change orientation through the wall), recent calculations challenge this view and show that they do have some chirality, i.e., the polarization rotates within them as in a magnetic Bloch wall (Lee et al., 2009). Therefore, when the domains are sufficiently small to be comparable to the thickness of the walls, the end result will be indeed something resembling the closure stripe configuration of Fig. 10. The existence of this domain wall chirality might seem surprising, but it was explained two decades ago by Houchmandzadeh, Lajzerowicz, and Salje (1991): If there is more than one order parameter involved in a ferroic (and perovskite ferroelectrics are always ferroelastic as well as ferroelectric), then the coupling introduces chirality. This, of course, is also true of magnetoelectric multiferroics (Seidel et al., 2009; Daraktchiev, Catalan, and Scott, 2010). The theoretical prediction of ferroelectric closurelike structures where domain walls meet an interface has been 

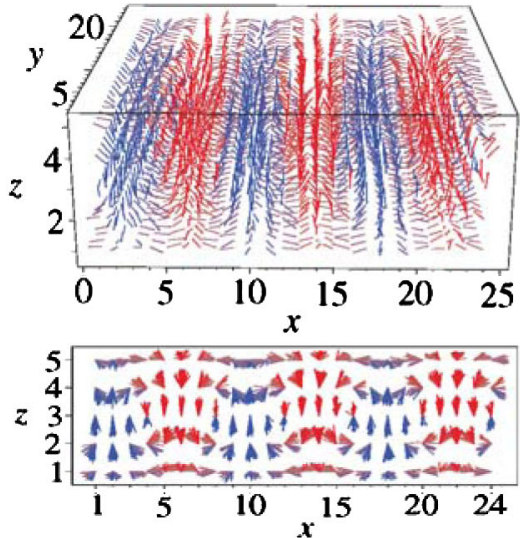
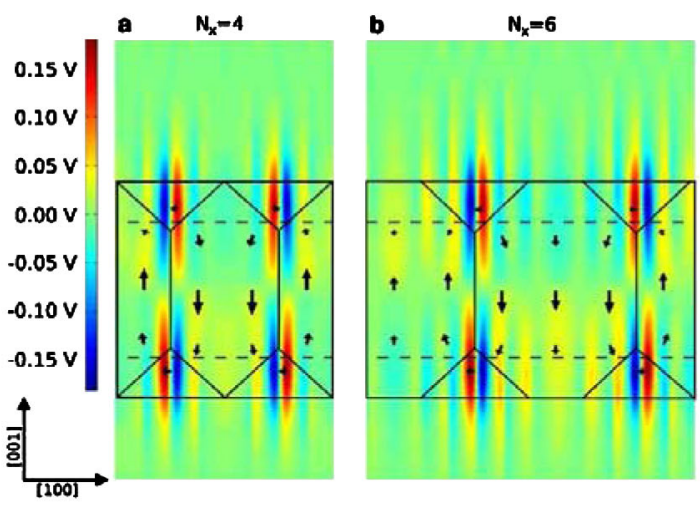

FIG. 10 (color online). Ferroelectric "closure stripes" predicted by atomistic simulations of ultrathin films. From Kornev, Fu, and Bellaiche, 2004 and Aguado-Fuente and Junquera, 2008.

experimentally confirmed by two different groups (Jia et al., 2011; Nelson et al., 2011) (see Fig. 11).

It is worth noticing here that the arrangements in Fig. 11 are not a classic fourfold closure structure, with four walls at $90^{\circ}$ converging in a central vertex. Instead, these domain structures should be seen as half of a closure quadrant. The bifurcation of a quadrant into two threefold vertices, with walls converging at angles of $90^{\circ}$ and $135^{\circ}$, was predicted by Srolovitz and Scott (1986); their schematic depiction of the bifurcation process is reproduced in Fig. 12. The reverse process of coalescence of two threefold vertices to form
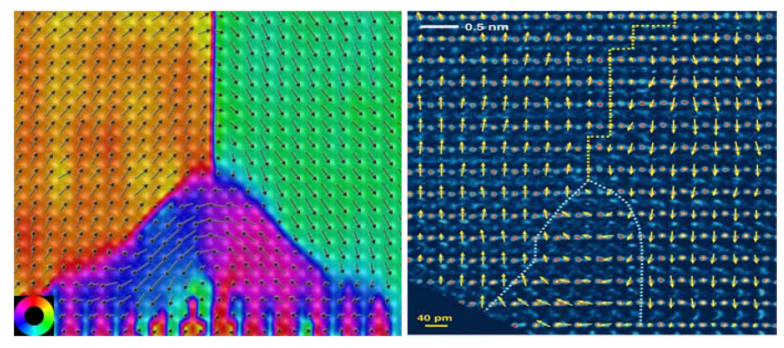

FIG. 11 (color online). Observation of closurelike polar arrangements at the junction between ferroelectric domain walls and an interface, for thin films of $\mathrm{BiFeO}_{3}$ (left) and $\mathrm{PbTiO}_{3}$ (right). Note that the wall angles are $135^{\circ}, 90^{\circ}, 135^{\circ}$, as in the Srolovitz-Scott model, not $120^{\circ}$. Adapted from Nelson et al., 2011 (left) and Jia et al., 2011 (right).
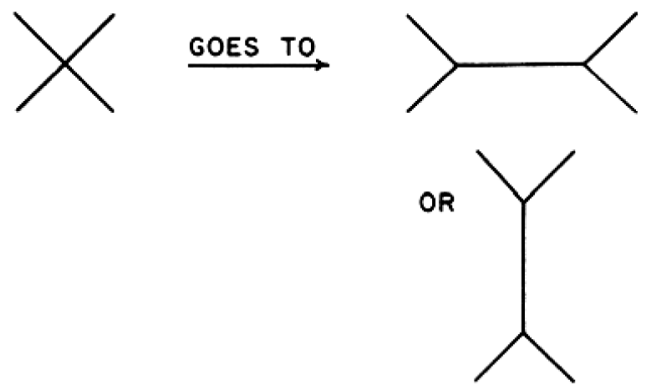

FIG. 12. A fourfold vertex in a $90^{\circ}$ quadrant is predicted by a Pott's model to bifurcate into two threefold vertices. From Srolovitz and Scott, 1986. one fourfold vertex, also predicted by Srolovitz and Scott, was recently observed in $\mathrm{BaTiO}_{3}$ by Gregg et al. (private communication). Vertices are a topological singularity closely related to vortices, the main difference being that a vortex implies flux closure, whereas a vertex is just a confluence of domain walls; some vertices are also vortices (e.g., the vertices of $90^{\circ}$ closure quadrants in ferromagnets and ferroelectrics), but others are not.

Vortices are frequently observed in ferromagnetic nanodots (Shinjo et al., 2000). At the vortex core, the spin must necessarily point out of the plane of the nanodot: This outof-plane magnetic singularity is extremely small, yet stable, and could therefore be useful for memories. Ferroelectric vortices are also theoretically possible (Naumov, Bellaiche, and Fu, 2004), and Naumov and co-workers predicted that such structures are switchable and should yield an unusually high density of "bits" for memory applications (Naumov et al., 2008).

So far, there is tantalizing experimental evidence for vortices in ferroelectrics (Gruverman et al., 2008; Rodriguez et al., 2009; Schilling et al., 2009). However, although vortices almost certainly appear as transients during switching (Naumov and Fu, 2007; Gruverman et al., 2008; Sene et al., 2009), it is difficult to observe static ferroelectric vortices, or even just closure structures, in conventional tetragonal ferroelectrics. This is because a simple quadrant arrangement generates enormous disclination strain (Arlt and Sasko, 1980) (see Fig. 13); for dots above a certain critical
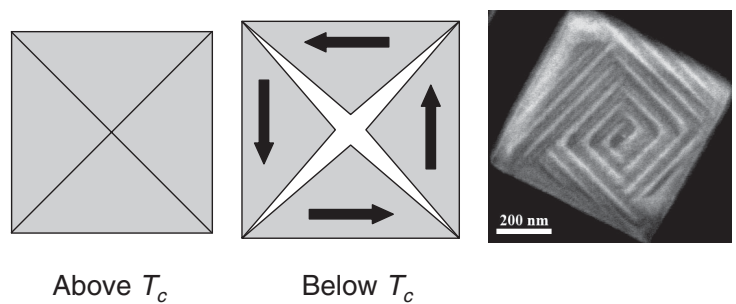

FIG. 13. (Left) Schematic illustration of the disclination stresses that are generated in the center of a closure structure of a tetragonal ferroelectric or ferroelastic; (right) experimental observation that ferroelastic stripes appear within the quadrants, probably in order to alleviate the stress. Adapted from Schilling et al., 2009. 
size, alleviation of associated stresses will be provided by the formation of ferroelastic stripe domains within each quadrant. A back-of-the-envelope calculation allows us to estimate the size at which the stripes will break the quadrant configuration. We do so by comparing the elastic energy stored within a single quadrant domain with the energy cost of a domain wall. The dimensions of the nanodot are $L \times L \times d, G$ is the elastic shear modulus, and $\varepsilon$ is the disclination strain, which is of the same order of magnitude as the spontaneous strain. The elastic energy density stored in a quadrant of volume $L^{2} d / 4$ is given by

$$
E_{\text {elastic }}=\frac{1}{2} G s^{2} \frac{L^{2} d}{4} .
$$

The energy cost of the first wall to divide the quadrant is the surface energy density of the wall $(\sigma)$ times the area of the new domain wall:

$$
E_{\text {wall }}=\sigma \frac{\sqrt{2}}{2} L d .
$$

When these two quantities are equal, the quadrant configuration stops being energetically favorable. By making $E_{\text {elastic }}=$ $E_{\text {wall }}$ we therefore obtain an approximate critical size

$$
L=4 \sqrt{2} \frac{\sigma}{G s^{2}},
$$

which, for the case of $\mathrm{BaTiO}_{3}(G=55 \mathrm{GPa}, \sigma=$ $3 \times 10^{-3} \mathrm{~J} / \mathrm{m}^{2}$, and $s=0.01$ ), gives a critical size of only $3 \mathrm{~nm}$. That small size explains why in larger ferroelectric nanocubes one observes a quadrantlike structure split by multiple ferroelastic stripes (Schilling et al., 2009) (see Fig. 13). More recently, ferroelectric flux closure has been confirmed in metadomain formations consisting of finely twinned quadrants (McQuaid et al., 2011).

Equation (7) shows that, in order to find a "pure" (nontwinned) ferroelectric quadrant structure, one will have to look for ferroelectrics with small spontaneous strain and high domain wall energy. $\mathrm{BiFeO}_{3}$ has a large domain wall energy (Catalan et al., 2008; Lubk, Gemming, and Spaldin, 2009) due to the coupling of polarization to antiferrodistortive and magnetic order parameters $\left(\mathrm{BiFeO}_{3}\right.$ is simultaneously ferroelectric, ferroelastic, ferrodistortive, and antiferromagnetic), while at the same time its piezoelectric deformation is small. That helps stabilize closure structures in this material (Balke et al., 2009; Nelson et al., 2011).

In purely magnetic materials, of course, vortex domains are well known and even their switching dynamics are now being studied, as illustrated in Fig. 14: Note that this figure shows that one can create magnetic vortex domains by repetitive application of demagnetizing fields to single-domain soft magnets. Similarly, Ivry et al. (2010) observed that application of depolarizing electric fields has a similar effect in ferroelectrics.

As mentioned earlier, a close relative of vortices and closure domains is what we call "vertex" domains. A vertex is the intersection between two or more domain walls in a ferroic. In the classic quadrant structure, the vertex is a fourfold intersection between $90^{\circ}$ domains, while in a needle domain the vertex is a twofold intersection. It is important to note that each of the domain walls intersecting the vertex is
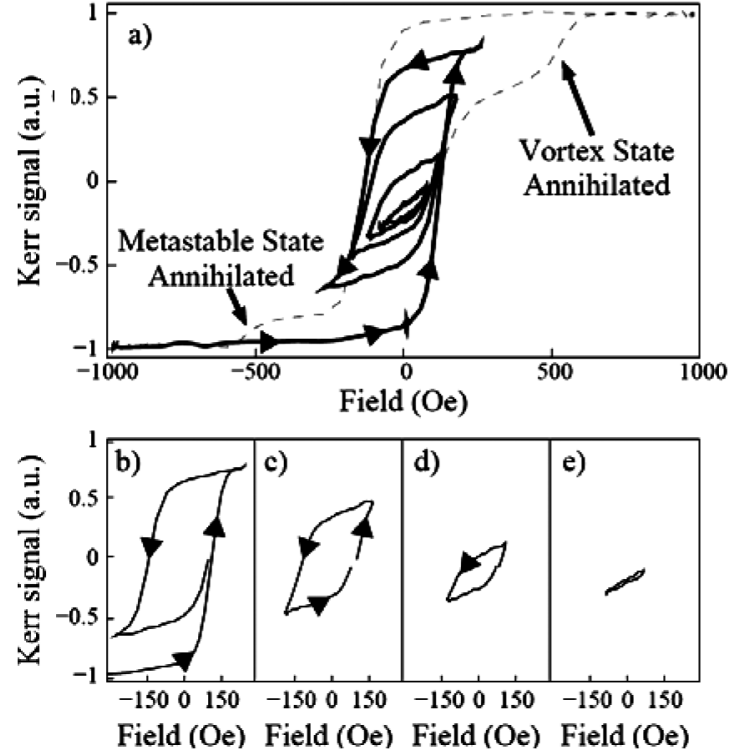

FIG. 14. Dynamic response of magnetic vortices, from the work of Cowburn's and co-workers: (a)-(e) Hysteresis curves showing the decay of a single-domain state into a vortex state via a series of minor hysteresis cycles. The entire decay process is shown in (a). The arrowed solid line indicates the direction of the transition from single domain to vortex state. The dashed line outlines the Kerr signal corresponding to the positive and negative applied saturation fields. The first three and the last demagnetizing cycles are displayed in separate panels; (b) first cycle, (c) second cycle, (d) third cycle, and (e) 18th cycle. From Ana-Vanessa, Xiong, and Cowburn, 2006.

equivalent through symmetry; that is, they cannot be different walls, such as (011) and (031), a point to which we return below. Using topological arguments, Janovec (1983) showed that the number $N$ of domain walls intersecting at the vertex is equal to the dimensionality of the order parameter. Janovec and Dvorak further developed the theory in a longer review in 1986. However, complicating the general theory of Janovec is the fact that several order parameters might coexist (as in multiferroic materials), and that the domains do not necessarily have the same energy.

The energetics and stability of vertex domains were analyzed by Srolovitz and Scott (1986) using Potts and clock models. They showed that fourfold vertices, such as are found in $\mathrm{Ba}_{2} \mathrm{NaNb}_{5} \mathrm{O}_{15}$ (Pan et al., 1985) can, in some materials, spontaneously separate into pairs of adjacent threefold vertices. There is an apparent paradox regarding closure domains between the group theoretic predictions of Janovec (1983) and Janovec and Dvorak (1986), and the clock-model calculations of Srolovitz and Scott (1986). In particular, Janovec states that threefold closure vertices are forbidden, whereas Srolovitz and Scott show that they may be energetically favored over fourfold vertices. The paradox is reconciled as follows: What Janovec specifically forbids are isolated threefold vertices with three $120^{\circ}$ angles between the domain walls. What Scott and Srolovitz predict is a separation of energetically metastable fourfold vertices into closely spaced pairs of threefold vertices; but these pairs each consist of one original $90^{\circ}$ angle between domain walls, and two $135^{\circ}$ angles along the line between the vertex pairs. Hence this 



FIG. 15 (color online). (Left) Twelvefold ferroelectric domain vertex in thiourea. From Brown and Hollingsworth, 1995. (Right) Sixfold vertex intersection between charge density wave domains in $2 \mathrm{H}-\mathrm{TaSe}_{2}$ (Chen, Gibson, and Fleming, 1982). Schematic in (a) and actual microscopy image in (b). These formations are topologically equivalent to the vertex domains $\mathrm{YMnO}_{3}$.

should properly be regarded as not a threefold domain vertex but rather a fourfold vertex that has separated slightly at its center. This phenomenon is analogous to the separation of the fourfold closure domains in $\mathrm{BaTiO}_{3}$.

Another example of vertex structures that does not satisfy the basic model of Janovec is that in thiourea inclusion compounds (Brown and Hollingsworth, 1995). In this case inclusions in a thiourea matrix result in large strains (strain coupling is not directly included in the Janovec model). The result, illustrated in Fig. 15, is a beautiful 12-fold vertex structure. Note that this is despite the fact that the order parameter is of $N=2$ dimensions in thiourea (Toledano and Toledano, 1987). The reason is strain. The domain cluster shown in thiourea is of domain walls of different symmetry, notably $\{130\}$ and $\{110\}$. Yet another example of domain wall vertices is provided by the charge density wave domains observed by Chen, Gibson, and Fleming (1982) in $2 \mathrm{H}-\mathrm{TaSe}_{2}$ (see Fig. 15); this system, with three spatial inplane orientations and + and - out-of-plane distortions, is equivalent to ferroelectric $\mathrm{YMnO}_{3}$. Both violate the simpler requirement described by Janovec (1983) that the number of domains $N$ at a vertex must equal the dimensionality $n$ of the order parameter and require incorporation of coupling terms plus energy considerations to determine the equilibrium structure, as done by Janovec et al. (1985, 1986). On a more general level, Saint-Gregoire et al. (1992) showed that domain wall vertex structure classifications consist of 36 twofold vertices with five equivalence classes, 96 fourfold vertices of ten classes, and 63 sixfold vertices of nine classes. It is notable that, even where walls carrying opposite $+P_{z}$ and $-P_{z}$ polarizations meet, the vertex can still have a polar point group (rod) symmetry, which is not intuitively obvious, but can be useful as these rods are analogous in this respect to the polar singularity at the core of a vortex. Note also that the socalled layer groups, such as $2{ }^{\prime}$, keep the central plane of a wall invariant, whereas the other groups do not. Rod groups can be chiral; for example, a regular sixfold vertex with symmetry $6_{z}{ }^{\prime}$ has a helical structure with polarization along $z$. There are two equivalent sixfold vertices with the same helicity by opposite polarization; the chirality does not dictate the polarization.

This situation is also encountered in multiferroic $\mathrm{YMnO}_{3}$. Although the sixfold vertices of $\mathrm{YMnO}_{3}$ were observed long ago by Safrankova, Fousek, and Kizhaev (1967), interest has been rekindled by more recent studies studying these formations in detail (Choi et al., 2010; Jungk et al., 2010) (see Fig. 16). The correct domain analysis requires the tripled unit cell of Fennie and Rabe (2005) for proper description, and not the simpler primitive cell proposed by Van Aken et al. (2004). The coupling of ferroelectricity to the other order parameters (antiferromagnetic and antiferrodistortive) yields the required dimensionality for the sixfold vertices to form. $\mathrm{YMnO}_{3}$ is also interesting because its domain walls are less conducting than the domains (Choi et al., 2010), which is the exact opposite of what happens in the other popular multiferroic, $\mathrm{BiFeO}_{3}$ (Seidel et al., 2009). The issue of domain wall conductivity is extensively discussed in later sections.

Recently, the functional properties of vertices and vortices are also starting to be studied. In the case of $\mathrm{BiFeO}_{3}$, for example, it has been found that the conductivity of ferroelectric vortices is considerably higher than that of the domain walls, which are in turn more conductive than the domains (Balke et al., 2011).

\section{G. Nanodomains in bulk}

Kittel's law implies that small domains can appear in small or thin samples, but nanodomains occur in some bulk compositions. Trivially, any material with a first-order phase transition will experience the nucleation of small nonpercolating domains above the nominal $T_{c}$. In the case of $\mathrm{BaTiO}_{3}$, these can occur more than $100^{\circ}$ above $T_{c}$ (Burns and Dacol, 1982). This, however, has little implication for the functional
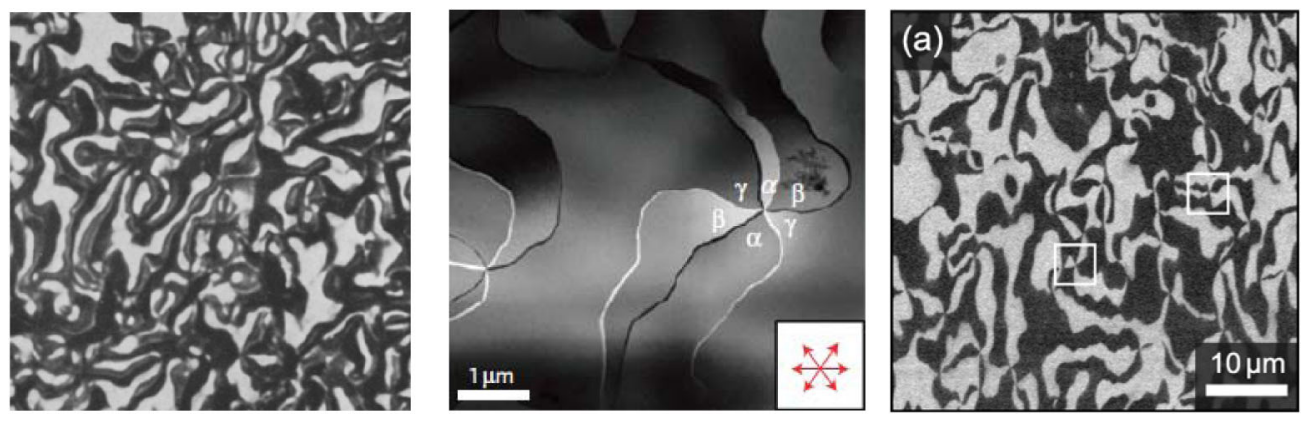

FIG. 16 (color online). Observation of sixfold vertices in domain ensembles of multiferroic $\mathrm{YMnO}_{3}$ : (left) from Safrankova, Fousek, and Kizhaev, 1967; (middle) from Choi et al., 2010, and (right) from Jungk et al., 2010. 
properties because the volume fraction occupied by such nanodomains is small. But there are other material families where nanodomains are inherent. These are nearly always linked to systems with competing phases and frustration, and the functional properties of nanoscopically disordered materials are often striking: colossal magnetoresistance in manganites, superelasticity in tweedlike martensites, and giant electrostriction in relaxors, to name a few.

Relaxors combine chemical segregation at the nanoscale and nanoscopic polar domains (Cross, 1987; Bokov and Ye, 2006). The key technological impact of these materials lies in their large extension under applied fields for piezoelectric actuators and transducers (Park and Shrout, 1997). Despite many papers on the basic physics of relaxor domains, a holistic theory is still missing. The presence of polar domains in the cubic phases of relaxors, where they are nominally forbidden, may be caused by flexoelectricity and internal strains due to local nonstoichiometry (Ahn et al., 2003). When mixed with ordinary ferroelectrics such as $\mathrm{PbTiO}_{3}$, or subjected to applied fields $E$, these nanodomains increase in size to become macroscopic (Mulvihill, Cross, and Uchino, 1995; Xu et al., 2006). As for the shape of the domains, in pure $\mathrm{PbZn}_{1 / 3} \mathrm{Nb}_{2 / 3} \mathrm{O}_{3}(\mathrm{PZN})$, the domain walls may be spindlelike (Mulvihill, Cross, and Uchino, 1995) or dendritic (Liu, 2004) but become increasing lamellar with increasing additions of $\mathrm{PbTiO}_{3}$. The condensed $\langle 110\rangle$ domain structure is stable in perovskites and rather unresponsive to fields $E$ along [111] (Xu et al., 2006), and the polar nanoregions arise from a condensation of a dynamic soft mode along [110], as shown via neutron spin-echo techniques (Matsuura et al., 2010). Multiferroic (magnetoelectric) relaxors also exist (Levstik et al., 2007; Kumar et al., 2009), but little is yet known about their domains.

From the perspective of this review, the key point about relaxors is that, since they are formed by nanodomains, they must have a large concentration of domain walls. It is therefore reasonable to expect that the domain walls contribute to the extraordinary electromechanical properties of these materials. Rao and $\mathrm{Yu}$ (2007) show that indeed there is an inverse correlation between domain size and piezoelectric behavior, and suggest that the linking mechanism is a fieldinduced broadening of the domain walls. On the other hand, domain walls may contribute not only by their static properties or broadening, but also by their dynamic response (motion) under applied electric fields, as suggested by the Rayleigh-type analyses of Davis, Damjanovic, and Setter (2006) and Zhang et al. (2010).

Polar nanodomains also exist in another nonpolar material, $\mathrm{SrTiO}_{3}$, which is important as it is the most common substrate for growing epitaxial films of other perovskites. $\mathrm{SrTiO}_{3}$ is cubic at room temperature, but tetragonal and ferroelastic below 105-110 K (Fleury, Scott, and Worlock, 1968). It is also an incipient ferroelectric whose transition to a macroscopic ferroelectric state is frustrated by quantum fluctuations of the soft phonon at low temperature; hence, the material is also called a "quantum paraelectric" (Muller and Burkard, 1979). By substituting the oxygen in the lattice for a heavier isotope, ${ }^{18} \mathrm{O}$, the lattice becomes heavier, and the phonon slows down and freezes at a higher temperature, causing a ferroelectric transition (Itoh et al., 1999). However, polar nanodomains have been detected even in the normal ${ }^{16} \mathrm{O}$ composition of $\mathrm{SrTiO}_{3}$ (Uesu et al., 2004; Blinc et al., 2005), and their local symmetry is triclinic and not tetragonal (Blinc et al., 2005). The ferroelectric phase of the heavyisotope composition is also poorly understood, but it has finely structured nanodomains reminiscent of those observed in relaxors (Uesu et al., 2004; Shigenari et al., 2006), while relaxorlike behavior has also been observed in $\mathrm{SrTiO}_{3}$ thin films (Jang et al., 2010). Again, the high concentration of domain walls concomitant with this fine domain structure shows important implications for functionality, since the domain walls of $\mathrm{SrTiO}_{3}$ are thought to be polar (Tagantsev, Courtens, and Arzel, 2001; Zubko et al., 2007). We also note that $\mathrm{SrTiO}_{3}$ at low temperatures has giant electrostriction comparable to that observed in relaxor ferroelectrics (Grupp and Goldman, 1997).

The above are examples of nanodomains that appear spontaneously in some special materials. But nanodomains can also be made to appear in conventional ferroelectrics by clever use of poling. Fouskova, Fousek, and Janoušek established that domain wall motion enhanced the electric-field response of ferroelectric material (Fouskova, 1965; Fousek and Janoušek, 1966), and domain engineering of crystals yields a piezoelectric performance far superior to that of normal ferroelectrics (Zhang et al., 1994; Eng, 1999; Bassiri-Gharb et al., 2007). However, a newer and more relevant twist is that even static domain walls may significantly enhance the properties of a crystal, due to the superior
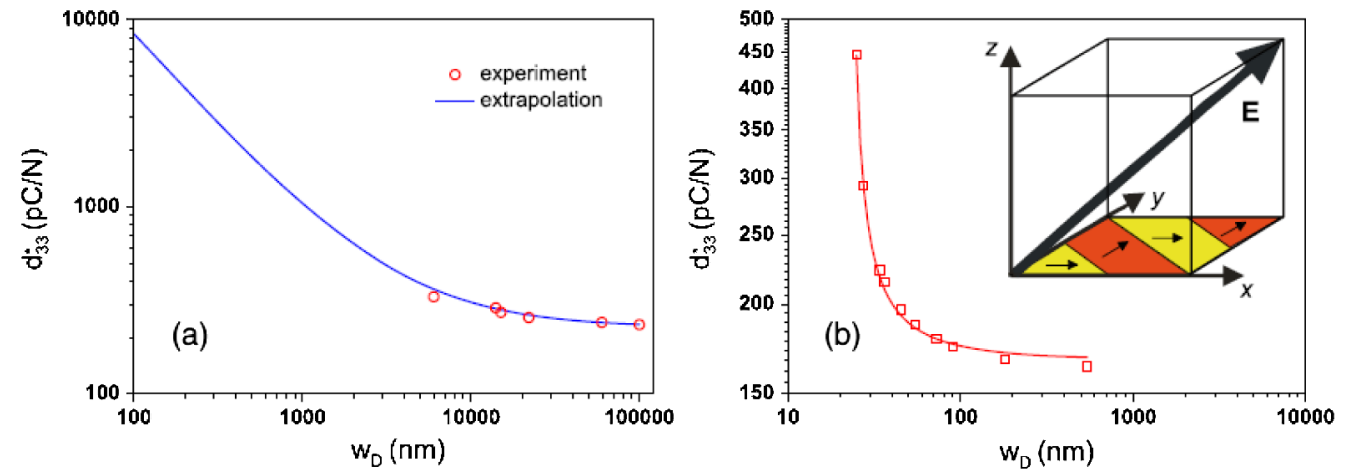

FIG. 17 (color online). Measurements and calculations relating decreased domain size (and thus increased domain wall concentration) to enhancement of piezoelectricity in $\mathrm{BaTiO}_{3}$ single crystals. The results suggest that the increased piezoelectric coefficient is due to the internal piezoelectricity of the domain walls. From Hlinka, Ondrejkovic, and Marton, 2009. 
piezoelectric properties of the domain wall itself (see Fig. 17). The concept of "domain wall engineering" was introduced by Wada and co-workers as a way to enhance the piezoelectric performance of ferroelectric crystals (Wada et al., 2006). At present, however, the size or even the exact mechanism whereby domain walls contribute to the piezoelectric enhancement is still a subject of debate (Hlinka, Ondrejkovic, and Marton, 2009; Jin, He, and Damjanovic, 2009).

\section{H. Why does domain size matter?}

The above is a fairly comprehensive discussion of the scaling of domains with device size and morphology. The main take-home message is that, as device size is reduced, domain size decreases in a way that can often be described by Kittel's law in any of its guises. Thus, the concentration of domain walls will increase. We can quantify this domain wall concentration fairly easily: Let us just rearrange the terms of the "universal" Kittel's law, [Eq. (2)]:

$$
\frac{\delta}{w}=\sqrt{\frac{\delta}{G d}} .
$$

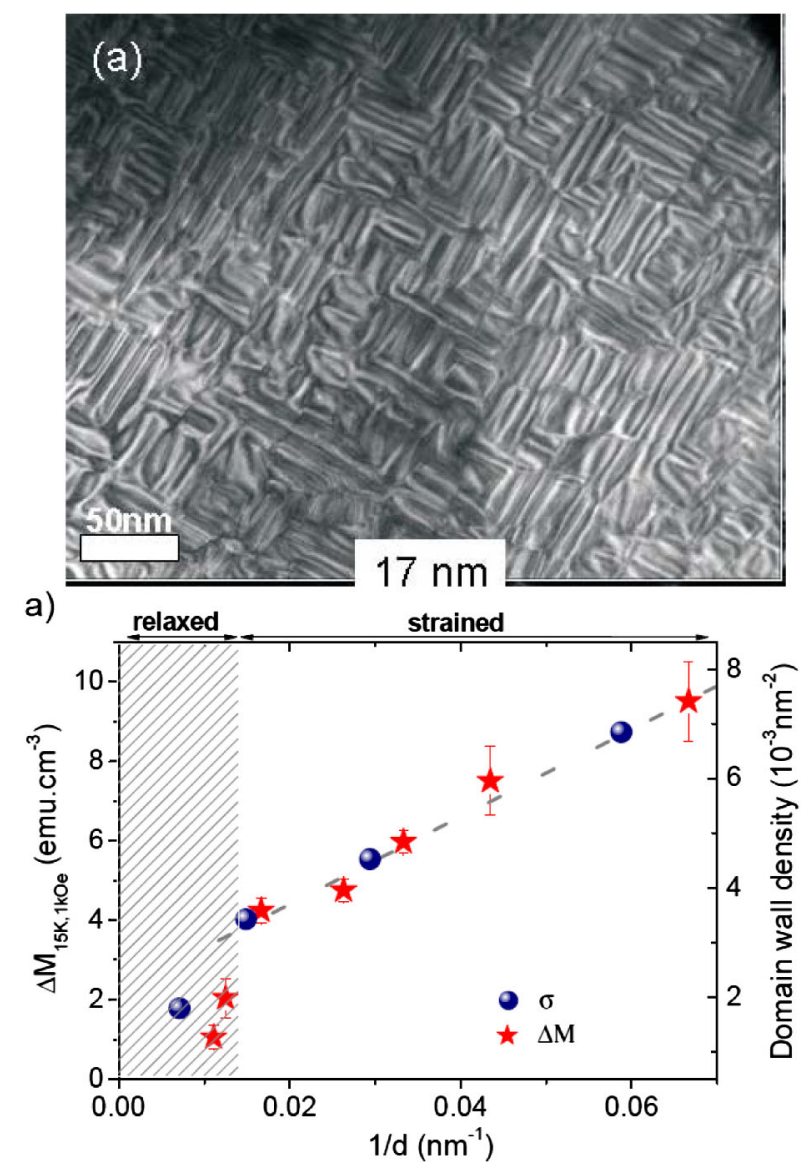

FIG. 18 (color online). (Top) The ferroelastic domains of orthorhombic $\mathrm{TbMnO}_{3}$ film grown on cubic $\mathrm{SrTiO}_{3}$ are so small ( $\approx 5 \mathrm{~nm}$ ) as to be comparable to the domain wall thickness, so that approximately $50 \%$ of the material is domain wall. (Bottom) The same authors report a strong correlation between inverse domain size (and thus domain wall concentration) and remnant magnetization in the films. From Daumont et al., 2009, 2010.
This equation shows that, as the film thickness $d$ decreases, the fraction $\delta / w$ (i.e., the fraction of the material that is made of domain walls) increases. Taking standard values for the domain wall thickness $\delta$ (typically $1-10 \mathrm{~nm}$ ), we can see that, for 100-nm-thick films, between $6 \%$ and $20 \%$ of the film's volume will be domain walls. Of course, as mentioned before, this percentage assumes that the surface energy is unscreened, so a correction factor must be applied when there is partial screening (the most general case). However, Eq. (8) is not completely unrealistic: Strain, for example, cannot be screened at all, and therefore ferroelastic domains (which in perovskite multiferroics tend to be ferroelectric and/or magnetic as well) can indeed be small. By way of illustration, consider the extremely dense ferroelastic domain structure in Fig. 18.

The high concentration of domain walls is important because domain walls not only have different properties from domains but, for specific applications, they can in fact be better (Wada et al., 2006). A sufficiently large number density of walls can therefore lead to useful emergent behavior in samples with nanodomains. This idea is barely in its infancy, but already there are hints that it could work. Daumont and co-workers, for example, report a strong correlation beween the macroscopic magnetization of a nominally antiferromagnetic thin film, and its concentration of domain walls (see Fig. 18).

The rest of this review will discuss the properties of domain walls, the experimental tools used to characterize them, and their possible technological applications.

\section{DOMAIN WALLS}

\section{A. Permissible domain walls: Symmetry and compatibility conditions}

Polar ferroics are those for which an inversion symmetry is broken: space inversion for ferrroelectrics or time inversion for ferromagnets. In these cases, domain walls separating regions of opposite polarity are possible, and they are called $180^{\circ}$ walls (in reference to the angle between the polar vectors on either side of the wall). $180^{\circ}$ walls tend to be parallel to the polar axis, so as to avoid head-to-head convergence of the spins or dipoles at the wall, as these are energetically costly due to the magnetic or electrostatic repulsion of the spins or dipoles. It is nevertheless worth mentioning that, although energetically costly, head-to-head $180^{\circ}$ walls are by no means impossible. $180^{\circ}$ head-to-head domains have been studied for decades in ferroelectrics. When they annihilate each other, large voltage pulses are emitted, called "Barkhausen pulses" (Newton, Ahearn, and McKay, 1949; Little, 1955); these voltage spikes are orders of magnitude larger than thermal noise. Most recently, head-tohead (charged) $180^{\circ}$ walls have been directly visualized using high-resolution transmission electron microscopy and found to be about 10 times thicker than neutral walls (Jia et al., 2008) (see Fig. 19). The difference in thickness between neutral and charged walls was historically first observed by Bursill, who noted the bigger thickness of the latter (Lin and Bursill, 1982; Bursill, Peng, and Feng, 1983; Bursill and Peng, 1986). According to Tagantsev (2010), this 

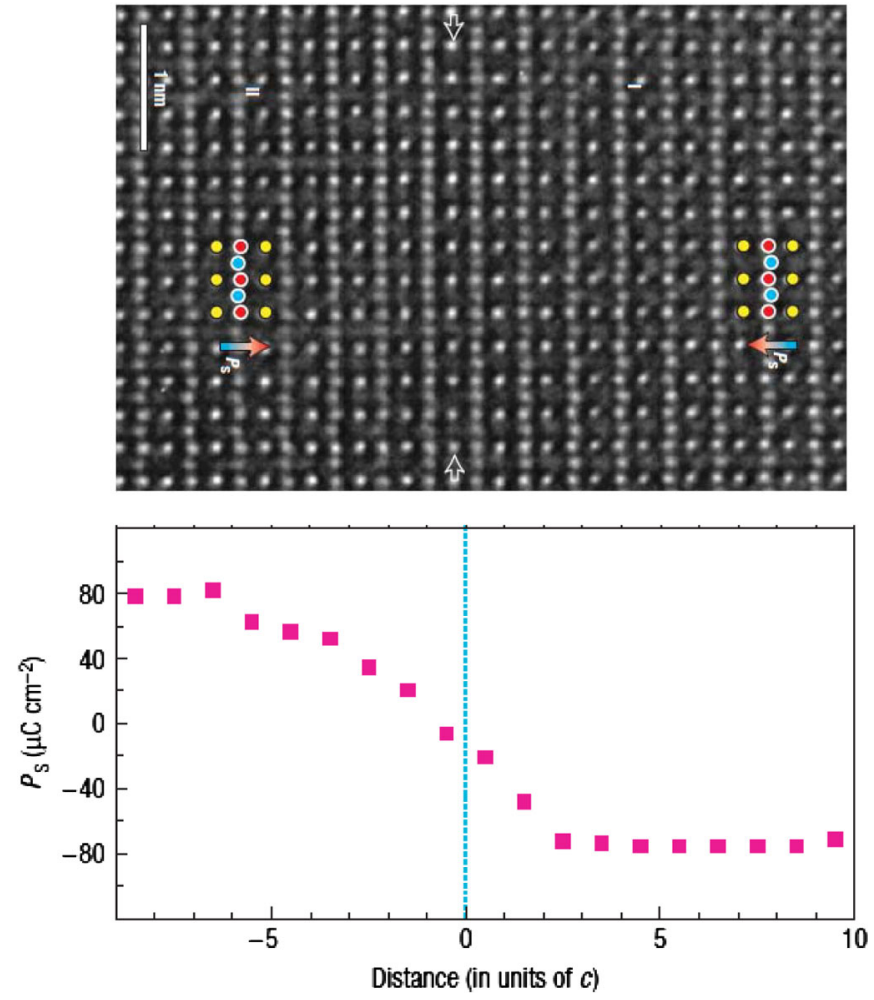

FIG. 19 (color online). High-resolution transmission electron microscopy image of a head-to-head charged domain wall in ferroelectric $\mathrm{Pb}(\mathrm{Zr}, \mathrm{Ti}) \mathrm{O}_{3}$. The domain wall is found to be approximately 10 unit cells thick, which is about 10 times thicker than for normal (noncharged) ferroelectric domain walls. From Jia et al., 2008, Nature Publishing Group.

increased thickness is due to the aggregation of charge carriers at the wall in order to screen the strong depolarizing field of the head-to-head dipoles. An interesting corollary to this observation is that the thickness of charged domain walls in semiconducting ferroelectrics will be different depending on whether they are head to head or tail to tail, due to the different availability of majority carriers; for example, in an $n$-type semiconductor, there is an abundance of electrons, and so head-to-head domain walls can be efficiently screened, while tail-to-tail cannot, meaning that the latter will be broader (Eliseev et al., 2011). Domain wall thickness is further discussed in Sec III.B.

The order parameter in ferroelastic materials is the spontaneous strain, which is not a vector but a second-rank tensor. Since the spontaneous strain tensor does not break inversion symmetry, purely ferroelastic materials do not have $180^{\circ}$ domains. Instead, a typical example of ferroelastic domains (also called twins) is the $90^{\circ}$ twins in tetragonal materials, where the spontaneous lattice strains in adjacent domains are perpendicular. In the case of $90^{\circ}$ domains, the locus of the wall is the bisector plane at $45^{\circ}$ with respect to the $\{001\}$ planes, because along these planes the difference between the spontaneous strains of the adjacent domains is zero, and thus the elastic energy cost of the wall is minimized (also known as the invariant plane). In the case of multiferroics that are simultaneously ferroelectric and ferroelastic, the polar compatibility conditions (e.g., no head-to-head polarization) must be added to the elastic ones. Fousek and Janovec did precisely that and compiled a table of permissible domain walls in ferroelectric and ferroelastic materials (Fousek and Janovec, 1969; Fousek, 1971). Whenever the domains are in an epitaxial thin film, there are further elastic constraints imposed by the substrate, as analyzed in the paper by Speck and Pompe (1994). A case study of permissible walls in epitaxial thin films of rhombohedral ferroelectricis was done by Streiffer et al. (1998), and this is relevant for $\mathrm{BiFeO}_{3}$ (space group $R 3 c$ ). In this case, the polar axis is the pseudocubic diagonal $\langle 111\rangle$, and domain walls separating inversions of one, two, or all three of the Cartesian components of the polarization are possible (these are called, respectively, $71^{\circ}$, $109^{\circ}$, and $180^{\circ}$ walls).

More generally, Aizu (1970) explained that the number of ferroic domain states, and thus of possible domain walls, is given by the ratio of the point group orders of the high- and low-symmetry phases, although Shuvalov, Dudnik, and Wagin (1985) argued that a higher number of domains ("superorientational states") may be permissible than given by the Aizu rule, as indeed observed in ferroelastic $\mathrm{YBa}_{2} \mathrm{Cu}_{3} \mathrm{O}_{7-\delta}$ (Schmid et al., 1988). Another important rule is given by Toledano (1974): It is necessary and sufficient for ferroelastic phase transitions that the crystal undergoes a change in crystal class (trigonal and hexagonal is regarded as a single superclass in this argument). The converse of that rule is that if there is no change in crystal class, then the material is not ferroelastic, and thus naturally there will not be any ferroelastic twin walls. Further restrictions apply to the type of domain walls that can exist in magnetoelectric materials (Litvin, Janovec, and Litvin, 1994).

Because these rules place strict conditions on what types of walls can exist in a ferroic, domain wall taxonomy can help clarify not only the true symmetry of the ferroic phase in a material, but also its relationship with the paraphase. An illustrative example is yet again $\mathrm{BiFeO}_{3}$ : The classification of its domain walls allowed the determination that the hightemperature $\beta$ phase (above $825^{\circ} \mathrm{C}$ ) was orthorhombic (Palai et al., 2008). The existence of orthorhombic twins was also used by Arnold et al. (2010) to argue that the highest-symmetry phase of $\mathrm{BiFeO}_{3}$ should be cubic, even though this cubic phase may be "virtual," as it probably occurs above the (also orthorhombic) $\gamma$ phase and beyond the melting temperature in most samples; however, Palai et al. (2010) found Raman evidence that a reversible orthorhombic-cubic transition exists in some specimens. The determination of this "virtual paraphase" is not trivial, since previously other authors had argued that the ultimate paraphase of $\mathrm{BiFeO}_{3}$ should be hexagonal $R \underline{3} c$, as in $\mathrm{LiNbO}_{3}$ (Ederer and Fennie, 2008), which is likely incorrect. The correct determination of the paraphase symmetry is of utmost importance because polar displacements are measured with respect to it.

\section{B. Domain wall thickness and domain wall profile}

Experimentally, domain wall thicknesses can be measured accurately only by using atomic-resolution electron microscopy techniques. Theoretical estimates can be obtained using a variety of methods, ranging from $a b$ initio calculations to phenomenological treatments or pseudospin models. We 
begin this section by offering a simple physical model that captures the essential physics of domain wall thickness.

The volume energy density of any ferroic material has at least two components: one from the ordering of the ferroic order parameter, and one from its gradient. Inside the domains, there is no gradient, and so only the homogeneous part of the energy has to be considered. The leading term in this energy is quadratic: $U=\frac{1}{2} \chi^{-1} P_{0}^{2}$ for ferroelectrics, $\frac{1}{2} \mu^{-1} M_{0}{ }^{2}$ for ferromagnets, and $\frac{1}{2} K s_{0}{ }^{2}$, where $K$ is the elastic constant and $s_{0}$ is the spontaneous strain. Meanwhile, inside the domain walls there is a strong gradient whose energy contribution is also quadratic, since it obviously cannot depend on whether you cross the wall from left to right or vice versa. Although the exact shape of the gradient is best described as a hyperbolic tangent, as a first approximation one can linearize the polarization profile across the wall as $P(x)=P_{0}[x /(\delta / 2)](-\delta / 2<x<-\delta / 2)$. In this linearized approximation, the gradient is simply the switched polarization (or magnetization, or strain) divided by the wall thickness, and hence the gradient energy can be approximated as $\frac{1}{2} k\left(2 P_{0} / \delta\right)^{2}$ (where $k$ is the gradient coefficient or "exchange" constant, since it measures the energy cost of locally changing the order parameter with respect to its nearest neighbors). Although in this discussion we use polarization as an example, all the equations and conclusions are valid for any other type of ferroic material.

The wall also has a contribution from the ferroic ordering, which changes across the wall: It is zero exactly at the center of the wall and it grows to reach the saturation value at the beginning and end of the wall. The energy density per unit area of the wall is obtained by integrating the two energy terms across its thickness. Hence,

$$
\begin{aligned}
\sigma & =\int_{-\delta / 2}^{\delta / 2}\left[\frac{1}{2} k\left(\frac{2 P_{0}}{\delta}\right)^{2}+\frac{1}{2} \chi^{-1} P(x)^{2}\right] d x \\
& =2 k \frac{P_{0}^{2}}{\delta}+\frac{1}{6} \chi^{-1} P_{0}^{2} \delta .
\end{aligned}
$$

The actual domain wall thickness will be that which minimizes this domain wall energy density; hence

$$
\frac{\partial \sigma}{\partial \delta}=0=-2 k \frac{P_{0}^{2}}{\delta^{2}}+\frac{1}{6} \chi^{-1} P_{0}^{2},
$$

which leads to

$$
\delta=2 \sqrt{3} \sqrt{k \chi}
$$

More elaborate phenomenological treatments are based on Landau theory (Zhirnov, 1959), the simplest potential being

$$
G=\frac{a}{2} P^{2}+\frac{b}{2} P^{4}+\frac{k}{2}\left(\frac{\partial P}{\partial x}\right)^{2} .
$$

Variational minimization of the order parameter across a domain wall at $x=0$ yields $\tanh (x / \lambda)$, with the correlation length $\lambda=2 P_{0}^{-1} \sqrt{2 k / b}$. Using $P_{0}=\sqrt{-a / b}$ and $\chi=$ $-1 / 2 a$ and defining the domain wall thickness as $\delta=2 \lambda$, we get

$$
\delta=2 \sqrt{-\frac{2 k}{a}}=4 \sqrt{\chi k} .
$$
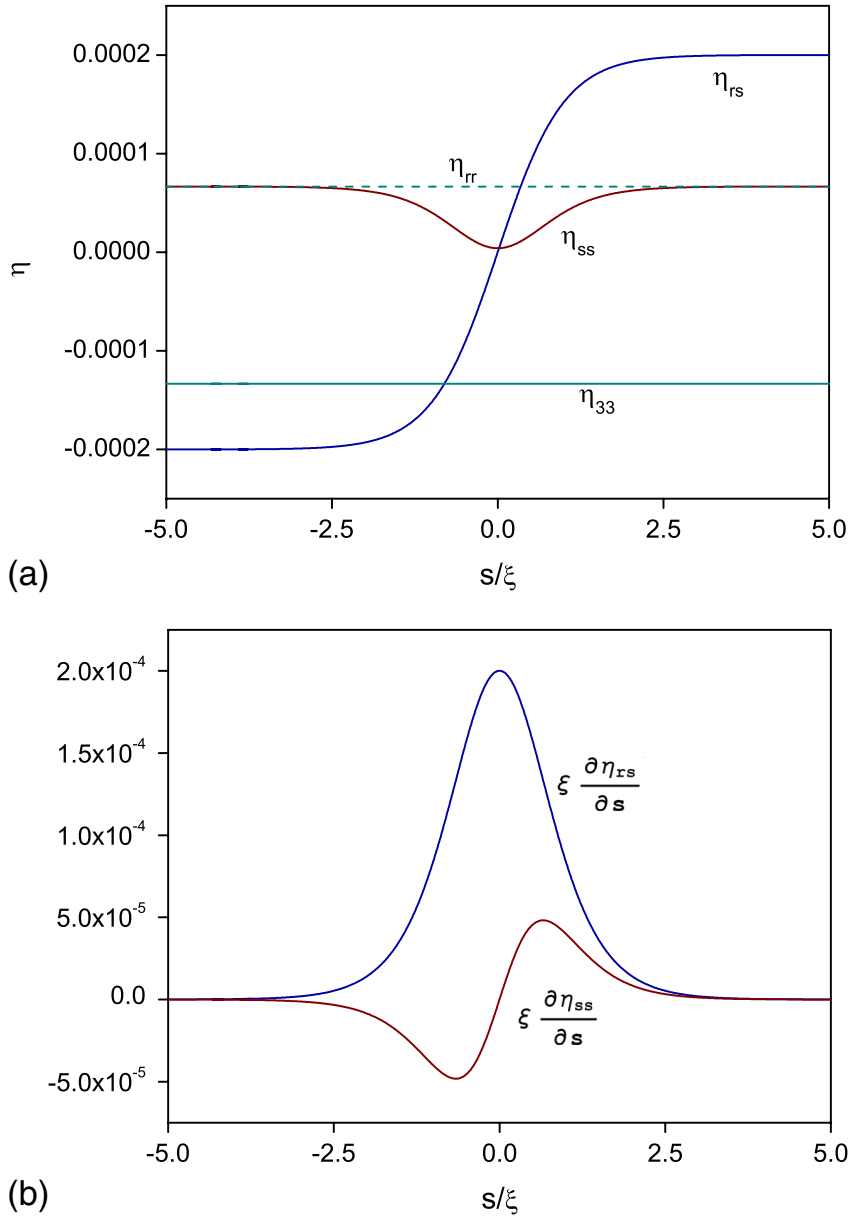

FIG. 20 (color online). (a) Ferroelastic strain components in the low-temperature domain walls of $\mathrm{SrTiO}_{3}$ and (b) flexoelectric polarizations caused by the strain gradients in the walls. From Zubko, 2008.

Note the remarkable similarity between Eqs. (11) and (13), despite the linear simplification assumed in the former.

An issue that appears to have been neglected by most [but not all, see Tagantsev, Courtens, and Arzel (2001)] phenomenological analyses of ferroelectric and ferroelastic domain walls is that the existence of large strain gradients at the walls must necessarily lead to considerable flexoelectricity inside them. Zubko (2008) performed some preliminary calculations for the gradients inside the ferroelastic domain walls of $\mathrm{SrTiO}_{3}$, using the strain profile calculated by $\mathrm{Cao}$ and Barsch (1990). The results are shown in Fig. 20. Assuming a ferroelastic correlation length $\xi \sim 4 \AA$ (one unit cell) and a flexoelectric coefficient of $10^{-8}$ (Zubko et al., 2007) (measured at room temperature and therefore smaller than the lowtemperature value), the flexoelectric polarization in the middle of the domain wall is of the order of $5 \mathrm{mC} / \mathrm{m}^{2}$ $\left(0.5 \mu \mathrm{C} / \mathrm{cm}^{2}\right)$, which is not negligible. This is only an approximate result, however, because the flexoelectric interaction must be incorporated into the domain wall structure calculations in a self-consistent manner, rather than a posteriori. We parenthetically note that strain gradients are significant at the nanoscale, and therefore flexoelectric effects are expected to be important. The large flexoelectric effects associated with nanodomains in ferroelectric thin 
films are currently being studied by several groups (Catalan et al., 2011; Lee et al., 2011; Lu et al., 2011).

At any rate, putting typical values into Eq. (13), it is found that ferroelectrics and ferroelastics have typical domain wall thicknesses in the range of $1-10 \mathrm{~nm}$, whereas ferromagnets have typically thicker walls in the range $10-100 \mathrm{~nm}$. This difference in thickness is not entirely surprising: Wall thickness is given by the competition between exchange and anisotropy (in ferromagnets) with the corresponding terms being dipolar energy and elastic anisotropy energy (in ferroelectrics). The exchange constant measures the energy cost of locally changing a spin, a dipole, or an atomic displacement (depending on the type of ferroic) with respect to its neighbors; in phenomenological treatments this was introduced as the energy cost of creating a gradient in the ferroic order parameter, exchange $=(k / 2)(\nabla P)^{2}$. If this energy is big, the ferroic will try to reduce the size of the gradient by increasing the thickness of the domain wall.

Likewise, the softness of the order parameter (its susceptibility) will also tend to broaden the walls: A material that has high susceptibility, dielectric, magnetic, or elastic, allows its order parameter to fluctuate more easily, meaning that broad domain walls, with a large number of unit cells departed from the equilibrium value, are still relatively cheap. Zhirnov (1959) offered a similar argument: The anisotropy measures the energy cost of misaligning the order parameter with respect to the crystallographic polar axes; if this energy is big, the ferroic will try to minimize the number of misaligned spins, dipoles, and strains by making the wall as thin as possible. Because both ferroelectricity and ferroelasticity are, at heart, structural properties, their anisotropy (arising from structural anisotropy such as, e.g., the tetragonality of a perovskite ferroelectric) will normally be larger than that of ferromagnets, and thus their wall thickness will be smaller. Hlinka (2008) and Hlinka and Marton (2008) have recently discussed the role of anisotropy on the domain wall thickness of the different phases of ferroelectric $\mathrm{BaTiO}_{3}$. The anisotropy argument is completely analogous to the susceptibility one, just by realizing that susceptibility is inversely proportional to anisotropy. It follows from the above that materials that are uniaxial and have small susceptibility should have far narrower domain walls than ferroics with several easy axes (so that they are more isotropic) and large permittivity; in particular, one may expect morphotropic phase boundary ferroelectrics to have anomalously thick domain walls, so that a significant volume fraction of the material may be made of domain walls. This is also the case for ultrasoft magnetic materials such as permalloys, or structurally soft materials such as some martensites and shape-memory alloys (Ren et al., 2009).

Domain wall thickness has traditionally been a contentious issue for ferroelectrics, where it has been hard to measure experimentally. The earliest electron microscopy measurements were reported by Blank and Amelinckx (1963), and they placed an upper bound of $10 \mathrm{~nm}$ on the $90^{\circ}$ wall thickness of barium titanate. Bursill and co-workers (Lin and Bursill, 1982; Bursill, Peng, and Feng, 1983, Bursill and Lin, 1986) used high-resolution electron microscopy to confirm that the domain walls of $\mathrm{LiTaO}_{3}$ and $\mathrm{KNbO}_{3}$ are indeed thin and atomically sharp in the case of $180^{\circ}$ walls.
Meanwhile, Floquet et al. (1997) combined high-resolution transmission electron microscopy with $\mathrm{x}$-ray diffraction to measure a width of $5 \mathrm{~nm}$ for the $90^{\circ}$ walls of $\mathrm{BaTiO}_{3}$. Shilo, Ravichandran, and Bhattacharya (2004) used atomic force microscopy (AFM) to measure the same type of walls in $\mathrm{PbTiO}_{3}$; although the tip radius of scanning probe microscopes (AFM, PFM) is typically $10 \mathrm{~nm}$, a careful statistical analysis allowed the intrinsic domain widths of ferroelectric and ferroelastic $90^{\circ}$ walls to be extracted; a wide range of thicknesses between 1 and $5 \mathrm{~nm}$ were recorded. They suggested that the intrinsic width is less than $1 \mathrm{~nm}$, and that the broadening observed in some measurements is due to the accumulation of point defects at the wall. The thickness of ferroelectric $180^{\circ}$ walls is harder to measure experimentally and is discussed in more detail in Sec. IV, but reliable theoretical predictions (Merz, 1954; Kinase and Takahashi, 1957; Padilla, Zhong, and Vanderbilt, 1996; Meyer and Vanderbilt, 2002) and recent measurements by Jia et al. (2008) indicated that they are atomically sharp, confirming the measurements of Bursill.

An interesting and still not fully resolved problem is that of the domain wall thickness in multiferroics. In materials with weak coupling, it is assumed that the two ferroic parameters have essentially independent correlation lengths and thus different thicknesses for the two ferroic parameters, even if the middle of the wall is shared (Fiebig et al., 2004). In the converse situation of one order parameter being completely subordinated to the other, e.g., a proper ferroelectric and an improper ferroelastic such as $\mathrm{BaTiO}_{3}$, or a proper magnet and an improper ferroelectric such as $\mathrm{TbMnO}_{3}$, it seems that the principal order parameter dictates a unique thickness of the shared domain wall, so that the ferroelectric domain walls of $\mathrm{TbMnO}_{3}$ are predicted to be as thick as those of ferromagnets (Cano and Levanyuk, 2010). In the intermediate case of two proper order parameters with moderate coupling, it seems that there will still be two correlation lengths for each order parameter, but each will be affected by the coupling; Daraktchiev, Catalan, and Scott (2010) have shown that the ferroelectric wall thickness in a magnetoelectric material with biquadratic coupling is

$$
\begin{aligned}
\delta_{\mathrm{MP}} & \equiv 2^{1 / 2} \sqrt{\left(\frac{\beta b \kappa}{2 \gamma \beta a-\alpha \gamma^{2}-\alpha \beta b}\right)} \\
& \cong \delta_{P}\left(1+\gamma \frac{a}{b \alpha}+O\left(\gamma^{2}\right)\right)
\end{aligned}
$$

where $\delta_{\mathrm{MP}}$ is the ferroelectric wall thickness in the magnetoelectric material, and $\delta_{P}$ is the ferroelectric wall thickness in the absence of magnetoelectricity. This is thicker than the walls of normal ferroelectrics and thus more magnetlike, which also agrees with the bigger width of the ferroelectric domains of BFO compared to those of normal ferroelectrics (Catalan et al., 2008).

\section{Domain wall chirality}

In magnetism, the spin is quantized, so it cannot change its magnitude across the wall. Instead, then, the magnetization reverses through rigid rotation of the spins. The rotation plane may be contained within the plane of the domain wall (Néel 
walls), or it may be perpendicular to it (Bloch walls). Néel and Bloch walls are generically termed as Heisenberg-like, or chiral. Ferroelectric polarization, on the other hand, is not quantized, so it is allowed to vary in magnitude. This can produce domain walls where the polarization axis does not change orientation but simply decreases in size, changes sign, and increases again. Such nonchiral domain walls are called Ising-like (see schematic of different types of walls in Fig. 21). In ferroelectrics, $180^{\circ}$ Ising walls should be favored against chiral walls for two reasons: First, the piezoelectric coupling between polarization and spontaneous strain means that rotating the polarization away from the easy axis has a big elastic cost. Second, there is also a large electrostatic cost, as any change in the polarization perpendicular to the domain wall will cause, via Poisson's equation, an accumulation of charge at the walls: $\Delta \mathbf{D}=\varepsilon \Delta \mathbf{P}=\rho$. It is worth mentioning that the above assumes that the dielectric constant is constant; when it is not, then the correct form of Poisson's equation is $\Delta \mathbf{D}=\varepsilon \Delta \mathbf{P}+\mathbf{E} \cdot \nabla \varepsilon=\rho$. The permittivity gradient can be important in thin films (Scott, 2000), and must be important also for the domain wall, where large structural changes take place within a narrow region.

For the above reasons, $180^{\circ}$ domain walls in ferroelectrics have been traditionally viewed as Ising-like. This common assumption, however, has recently been challenged by Lee et al. (2009) and Marton, Rychetsky, and Hlinka (2010), who show that ferroelectric $180^{\circ}$ domain walls of perovskite ferroelectrics can be at least partially chiral. The fact that chirality can appear in a system where none would be expected was examined by Houchmandzadeh, Lajzerowicz, and Salje (1991). They showed that, whenever there are two order parameters involved (as in any multiferroic system), the coupling between them can induce chirality at the domain walls. Perovskite ferroelectrics are multiferroic, because they are both ferroelectric and ferroelastic. While their $180^{\circ}$ walls tend to be seen as purely ferroelectric, they nevertheless have an elastic component, because the suppression of the polarization inside the wall affects its internal strain (Zhirnov, 1959).

Domain walls in $\mathrm{BiFeO}_{3}$ are also multiferroic, and in a big way, ferroelectricity, ferroelasticity, antiferromagnetism, and


FIG. 21 (color online). (a) Ising wall, (b) Bloch wall, (c) Néel wall, and (d) mixed Ising-Néel wall. Recent calculations show that domain walls in perovskite ferroelectrics tend to be of mixed character. From Lee et al., 2009.

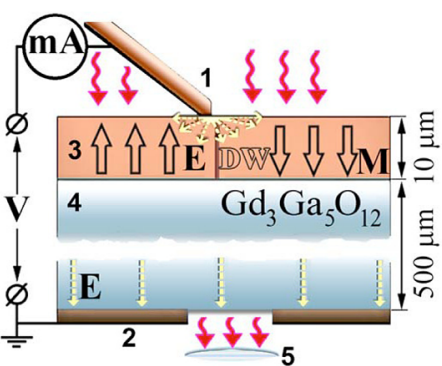

(a)

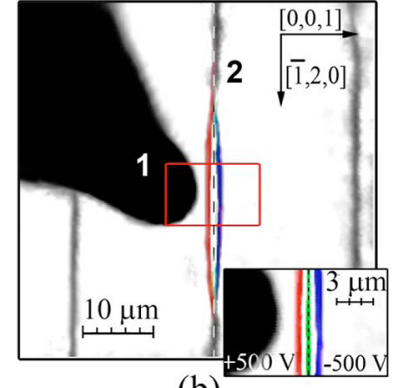

(b)
FIG. 22 (color online). (a) Logginov et al. applied voltage pulses to a sharp tip in the vicinity of a ferromagnetic Néel wall in a magnetic garnet. (b) The wall was observed to move toward or away from the tip depending on the polarity of the voltage, suggesting that the domain wall is electrically polarized even though the garnet itself is a nonpolar material. The domain wall polarization is caused by the spin spiral inherent to the Néel wall. From Logginov et al., 2008.

antiferrodistortive octahedral rotations all occur in this material. It is therefore not surprising that the domain walls of this material are found to be chiral (Seidel et al., 2009). Unlike in normal ferroelectrics, the rotation of the polar vector is quite rigid, meaning that the component of the polarization perpendicular to the domain wall is not constant. This polar discontinuity means that there is charge density at the walls (see Poisson's equation above). In order to screen this charge density, charge carriers aggregate to the wall, and this carrier increase has been hypothesized to be a cause for the increased conductivity at the domain walls of $\mathrm{BiFeO}_{3}$ (Seidel et al., 2009; Lubk, Gemming, and Spaldin, 2009). The issue of domain wall conductivity is discussed in greater detail in Secs. III.F and V.F.1.

Chirality has important consequences for magnetoelectric materials. Magnetic spin spirals can by themselves cause ferroelectricity: indeed, a spin spiral arrangement is known to cause weak ferroelectricity in some multiferroics (Newnham et al., 1978; Mostovoy, 2006; Cheong and Mostovoy, 2007). The relationship between spin helicity and polarization is valid not just for bulk but also for the local spin arrangement inside a domain wall; thus, ferromagnetic Néel walls are expected to be electrically polarized. Experimental evidence for this was provided by Logginov et al. (2008), who applied a voltage to an AFM tip placed near the ferromagnetic domain wall of a garnet, and observed the domain wall to shift its position in response to the voltage (see Fig. 22). Since the garnet is itself centrosymmetric, the piezoelectric response of the domain wall was attributed to its spin spiral.

\section{Domain wall roughness and fractal dimensions}

Irregular domain walls have been studied in thin films of ferromagnets (Lemerle et al., 1998), ferroelectrics (Tybell et al., 2002; Paruch, Giamarchi, and Triscone, 2005), and multiferroics (Catalan et al., 2008). Quantitatively, the irregular morphology can be characterized by a roughness coefficient (see Fig. 23), which describes the deviations $(u)$ from a straight line (the ideal domain wall) as the length of 


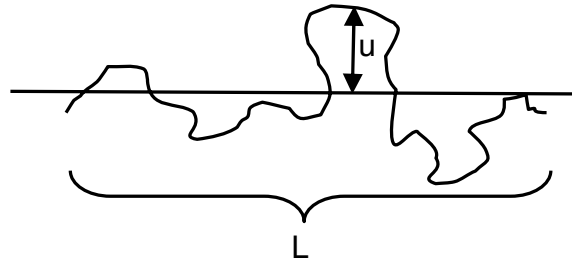

(a)

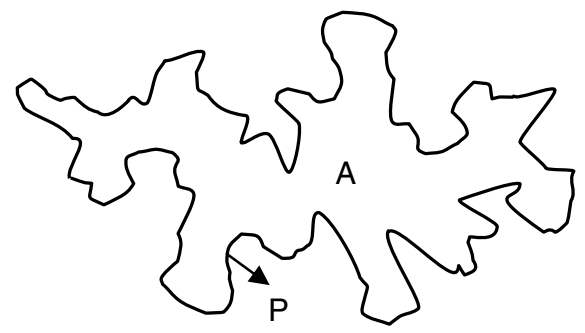

(b)
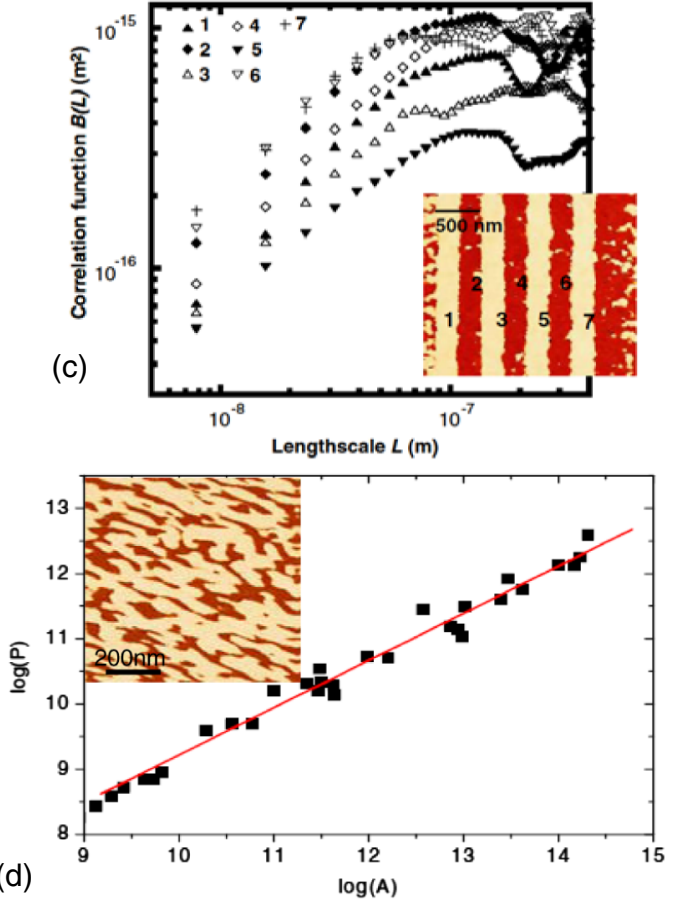

FIG. 23 (color online). (a) The probability of having a deviation $(u)$ from the straight line increases with the distance $d$ between two points of the wall, resulting in a power law relationship between the size of the wall and its horizontal length. By the same token, if the domain wall closes in on itself (b), the perimeter will not increase as the square root of the area (as would be the case for a smooth circular domain), but as $P \propto A^{H / 2}$, where $H$ is the Hausdorff dimension. (c) Measurement of domain wall roughness in PFM-written ferroelectric domain walls of $\mathrm{BiFeO}_{3}$ thin films and (d) measurement of the Hausdorff dimension in spontaneous domains of the same $\mathrm{BiFeO}_{3}$ films. Panels (c) and (d) partially adapted from Catalan et al., 2008.

wall is increased (Lemerle et al., 1998; Paruch, Giamarchi, and Triscone, 2005). The wandering deviation $u$ increases with the distance traveled along the wall, resulting in a power law dependence of the correlation coefficient, $B(L) \equiv$ $\left\langle\overline{[u(x+L)-u(x)]^{2}}\right\rangle \propto L^{2 \zeta}$, where $\zeta$ is the roughness exponent.

If the domain wall closes in on itself, forming a "bubble" domain, the roughness coefficient of the wall becomes a direct proxy for the Hausdorff dimension, which relates the area contained within the domain $(A)$ to the domain wall perimeter (see Fig. 23); thus, films with rough walls have fractal domains in the sense that the perimeter does not scale as the square root of the area, but as the square root of the area to the power of the Hausdorff dimension, $P \propto A^{H / 2}$ (Rodriguez et al., 2007a; Catalan et al., 2008).

Since domain size is dictated by the competition between the domain energy (proportional to the area of the domain) and wall energy (proportional to the domain perimeter) it is quite natural that the scaling of the domain size should reflect the Hausdorff dimension of the domains, or, equivalently, the roughness coefficient of the walls. Catalan et al. (2008) showed that, when the domains are fractal, the Kittel law must be modified as $w=A^{\prime} t^{H_{\perp} /\left(3-H_{l l}\right)}$, where $A^{\prime}$ is a constant, $t$ is the film's thickness, and $H_{\perp}$ and $H_{\|}$are the perpendicular and parallel (out-of-plane and in-plane with respect to the film's surface) Hausdorff dimensions of the walls. When both these dimensions are 1 (i.e., smooth walls), the classic Kittel exponent $\left(\frac{1}{2}\right)$ is recovered. In the particular case of BFO films, the dimension was found to be 1.5 in the in-plane direction, and 2.5 in total, consistent with domain walls that meander in the horizontal direction but are completely straight vertically, much like hanging curtains. This is fully expected in ferroelectric $180^{\circ}$ walls, as any vertical bend would incur in a strong electrostatic cost due to Poisson's equation. The fractional dimensionality has also been observed in studies of switching dynamics (Scott, 2007), as discussed later in this section.

In a perfect system, the domain wall energy cost is minimized by minimizing the wall area, i.e., by making the wall as smooth as possible. Whenever domain walls are rough, then, it is because they are being pinned by defects in the lattice. The upshot of this is that the roughness of a domain wall contains information about the type of defects present in the sample (Natterman, 1983). Specifically, the theoretical roughness coefficient in a random bond system is $\zeta=2 / 3$ for a linelike domain wall (Huse, Henley, and Fisher, 1985; Kardar and Nelson, 1985), and this has been experimentally verified for ultrathin ferromagnetic films (Lemerle et al., 1998). In the more general case, it is $\zeta=4-D / 5$ (Lemerle et al., 1998). Random bond systems can be viewed as systems with a variable depth of the double well. If the asymmetry of the double well changes, then one speaks of random field systems, for which the roughness coefficient is $\zeta=4-D / 3$ (Fisher, 1986; Tybell et al., 2002), where $D$ is the dimensionality of the wall, which can be fractional. For ferroelectric thin films the roughness coefficient was found to be 0.26 , consistent with the random bond system of dimensionality $D=2.7$ (Paruch, Giamarchi, and Triscone, 




FIG. 24. Ferroelectric domain wall speed as a function of applied electric field for films of various thicknesses. The critical exponent was found to be $\mu=1$, characteristic of creep. From Tybell et al., 2002.

2005). In multiferroic $\mathrm{BiFeO}_{3}$, the roughness was larger, $\zeta=0.56$ (Catalan et al., 2008).

Since the roughness of the walls arises directly from the local pinning by defects, and pinning slows down the motion of the domain walls, it is natural to relate the roughness of the domain walls to their dynamics. This has been done both for ferromagnetic films (Lemerle et al., 1998) and for ferroelectric films (Tybell et al., 2002; Paruch, Giamarchi, and Triscone, 2005). The domain wall velocity is characterized by an exponent that, similar to the roughness exponent, is also directly related to the type of pinning defects in the samples. Specifically, the velocity of the wall is

$$
v=v_{0} \exp \left[-\frac{U}{k T}\left(\frac{F_{\text {crit }}}{F}\right)^{\mu}\right]
$$

where $F$ and $F_{\text {crit }}$ are the applied and critical fields (magnetic or ferroelectric) of the sample, $U$ is an activation energy, and $\mu$ is the critical exponent, which is related to the roughness exponent by $\mu=(D+2 \zeta-2) /(2-\varsigma)$ (Lemerle et al., 1998). The value of $\mu$ depends on whether the domain wall motion proceeds by creep or by viscous flow; in ferroelectric thin films $\mu=1$ was measured, consistent with a creep process (see Fig. 24).

The study of the switching dynamics in ferroelectric thin films generally yields an effective dimensionality that is not integer but fractional. In early switching studies Scott et al. (1988) found from fits to the Ishibashi theory that dimensionality of the domain kinetics was often $D=2.5$ (approximately). At the time it was not clear whether this was a physical result or an artifact of the Ishibashi approximations (especially the simplifying assumption that wall velocities $v$ were independent of domain radius $r$-actually $v$ varies as $1 / r$ ). However, more recent studies (Scott, 2007) indicate that $D=2.5$ is physically correct; Scott also calculated by interpolation the critical exponents in mean field for $D=2.5$ and found, for example, that the order parameter exponent $\beta=\frac{1}{4}$ for a second-order transition, compared with $\beta=\frac{1}{2}$ for bulk $D=3$. Since this is the same $\frac{1}{4}$ exponent as in a bulk tricritical transition, second-order transitions for $D=2.5$ may be mistaken as tricritical.

\section{E. Multiferroic walls and phase transitions inside domain walls}

The idea that domain walls have their own symmetry and properties is not new. Shortly after Néel hypothesized the existence of antiferromagnetic domains (Néel, 1954), Li (1956) showed that such walls would have uncompensated spins that could account for the weak ferromagnetism measured in $\alpha-\mathrm{Fe}_{2} \mathrm{O}_{3}$. An important and often overlooked aspect of Li's classic model is that the size of the uncompensated moment at the wall is inversely proportional to the wall thickness. This, to some extent, is trivial: An atomically sharp antiphase boundary should have a fully uncompensated pair of moments (see Fig. 25), whereas in a broad domain wall the gradual change means that only the fractional difference between nearest neighbors is uncompensated. Although it has not been explicitly stated anywhere, a natural corollary is that the domain walls of antiferroelectrics should be ferroelectric, or at least pyroelectric. One must bear in mind that the walls of ferroelectrics are atomically sharp, as discussed above, so antiferroelectric domain walls are expected to be close to perfect antiphase boundaries, although we know of no studies of domain wall thickness in antiferroelectrics.

In the case of multiferroics, the interplay between the symmetries of all the phases involved is more complex and can lead to rich behavior. Privratska, Janovec, and others made a theoretical survey of which properties are allowed inside the domain walls as a function of the space group of the ferroic material. Based on this, they predicted that the domain walls of ferroelastics can be polar (Janovec, Richterová, and Privratska, 1999), as confirmed by atomistic calculations for

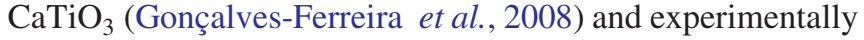
inferred for $\mathrm{SrTiO}_{3}$ (Zubko et al., 2007). Privratska and Janovec (1997, 1999), and Privratska (2007) also predicted that there can be net magnetization inside the ferroelectric

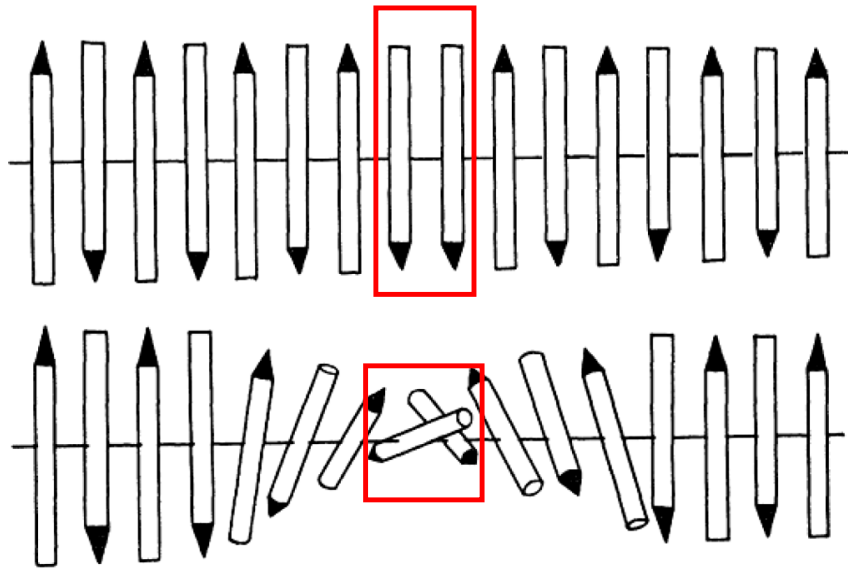

FIG. 25 (color online). The relative heights of the boxes illustrate how a sharp antiphase boundary must have a net magnetization and polarization in its center that is bigger than that of a broad domain wall such as a chiral wall. Adapted from Li, 1956. 
domain walls of multiferroics. Among the symmetries where this domain wall magnetization is allowed is the space group $R 3 c$ (Privratska and Janovec, 1999) (i.e., that of $\mathrm{BiFeO}_{3}$ ).

Symmetry analyses are not quantitative. The knowledge that a property is symmetry allowed is essential, but one still needs to know how big that property is. This quantitative analysis can be achieved using phenomenological approaches such as those pioneered by Lajzerowicz and Niez (1979), who were the first to realize that it was possible for domain walls to undergo their own internal phase transitions. This is possible because the free energy inside the wall is different from that inside the domains: Inside the domains the order parameter is homogeneous and there are no gradients, whereas inside the domain walls the order parameter is suppressed and there are strong gradients. Since the free energy of the wall is different, its thermodynamic properties must also be different, and hence its thermal evolution and phase transitions may also be different.

This idea becomes, of course, more interesting when several order parameters are involved. These order parameters may be present at finite temperature (as in multiferroic materials), or they may be suppressed or "latent" (have low or "negative" critical temperatures) but still may be able to manifest inside the domain walls. The phenomenon of emerging order parameters inside a wall was first explained by Houchmandzadeh, Lajzerowicz, and Salje (1991) in the context of ferroelastic materials. They realized that if a secondary ferroic order is latent (suppressed) due to a positive coupling energy to the primary order parameter, it will be able to emerge wherever the primary order parameter is zero, i.e., in the middle of the domain wall. For example, it is known that, in perovskites, rotations of the oxygen octahedra are normally opposed to ferroelectric polarization. Hence, where such rotations are suppressed, polarization may be able to emerge, as was theoretically calculated for the antiferrodistortive antiphase boundaries of $\mathrm{SrTiO}_{3}$ (Tagantsev, Courtens, and Arzel, 2001). Given that $\mathrm{BiFeO}_{3}$ is known to also have strong octahedral rotations (Megaw and Darlington, 1975) that oppose the polarization (Dupé et al., 2010), it seems eminently plausible that the ferrodistortive antiphase boundaries of this material also have a polar enhancement.

Daraktchiev, Catalan, and Scott (2010) studied in some detail the analytical solutions for domain walls in multiferroics with biquadratic coupling between the order parameters, $\gamma P^{2} M^{2}$; the biquadratic coupling was chosen because (i) it is the smallest power that is symmetry allowed for all materials (the coupling term places no constraint on inversion of either order parameter), and (ii) an effective biquadratic interaction will always be present when strain mediates the coupling, since electrostriction couples strain to the square of polarization while magnetostriction couples strain to the square of magnetization. Strain coupling terms are of course large in ferroelectrics, and therefore they will always be important for multiferroics. Another important aspect of this biquadratic coupling that perhaps has not been emphasized enough is that, because it is even, the solutions must also be even, meaning that any emerging parameter inside the wall will have at least two equally stable polarities and may be switchable between them. This can be seen, for example, in Fig. 26, which shows that there are two equivalent least-energy
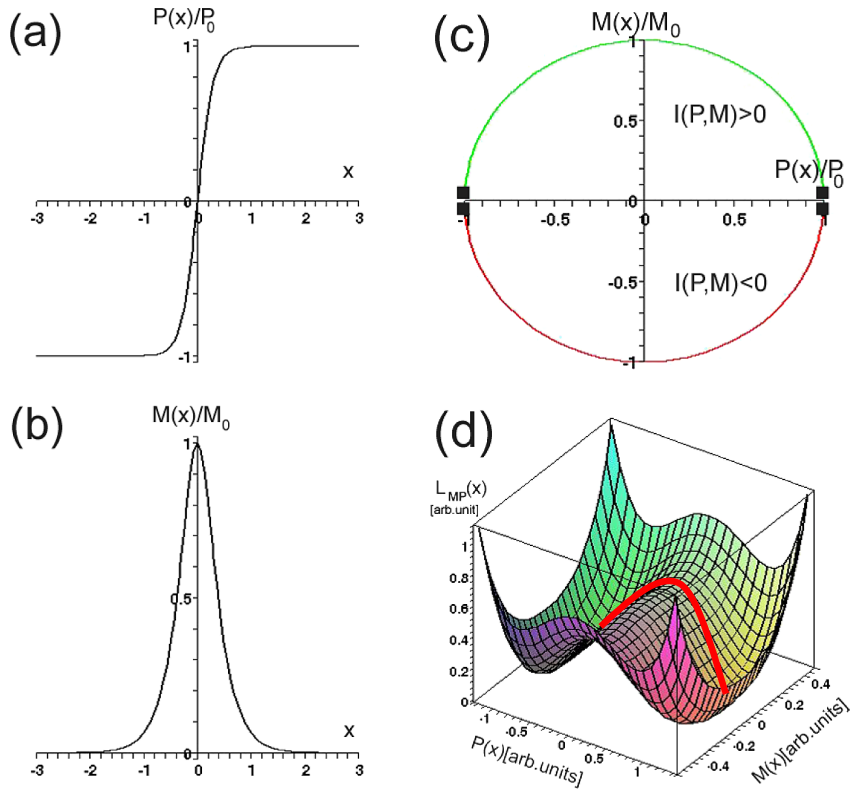

FIG. 26 (color online). (a) Ferroelectric polarization profile across the wall, (b) magnetization profile across the wall, (c) relationship between the order parameters across the wall, showing that the magnetization can be either positive or negative, and (d) free-energy landscape, showing one of the two least-energy trajectories connecting the two ferroelectric polarities through one of the saddle points at $M \neq 0$. Adapted from Daraktchiev, Catalan, and Scott, 2010.

trajectories connecting the ferroelectric double well through two saddle points at $+M$ and $-M$, and thus there are two possible magnetic polarities for the wall. Experimentally, Pyatakov et al. (2011) demonstrated the converse situation by showing switching of the ferroelectric polarity inside a magnetic domain wall. The exact phenomenology, of course, depends on the sign and symmetry of the coupling elements, and the possibilities are far too numerous to be described here, but the basic principle is always the same: Start with the Landau expansion of the free energy and examine the consequence of forcing one of the order parameters to be zero, as in the middle of the domain wall. In this context, it is useful to also look at the recent work of Marton, Rychetsky, and Hlinka (2010), who found new phases with enhanced electromechanical properties inside the domain walls of a typical perovskite ferroelectric such as $\mathrm{BaTiO}_{3}$.

So far group-symmetry arguments and phenomenological (thermodynamic) models have been mentioned, but there are also microscopic calculations for some systems. For example, Goltsev and others calculated the profile of the magnetization across the domain walls of $\mathrm{YMnO}_{3}$ (Goltsev et al., 2003; Fiebig et al., 2004). Meanwhile, Lubk, Gemming, and Spaldin (2009) calculated the octahedral rotations, polarization profile, and band gap across the domain walls of $\mathrm{BiFeO}_{3}$, and Gonçalves-Ferreira et al. (2008) calculated the polar displacements inside the ferroelastic walls of $\mathrm{CaTiO}_{3}$. Generally, the reduced thickness of ferroelectric and ferroelastic walls means that they are computationally affordable for first-principles calculations, whereas the broader magnetic walls tend to require analytical approaches or finite-element 
and molecular dynamics calculations. Multiferroic walls fall somewhere in between in terms of thickness, although we suspect that the magnetic part of their behavior will always be more difficult to compute using first-principles approaches.

\section{F. Domain wall conductivity}

The changes in structure (and as a consequence electronic structure) that occur at ferroelectric (multiferroic) domain walls can thus lead to changes in transport behavior. Indeed, domain wall (super)conductivity was studied by Aird and Salje (1998). Reducing $\mathrm{WO}_{3}$ with sodium vapor, they observed preferential doping along the ferroelastic domain walls. Transport measurements showed superconductivity, while magnetic measurements did not; this suggested that the superconductivity was located only at the domain walls, which provided a percolating superconductive path while occupying a small volume fraction of the crystal. Later, Bartels et al. (2003) used a conducting-tip scanning probe microscope to show the converse behavior: The domain walls of a calcium-doped lead orthophosphate crystal were found to be more resistive than the domains.

The above effects relied on preferential doping along domain walls, but the differential domain wall conductivity was reproduced also in undoped multiferroics, although with different transport behavior: The domain walls of $\mathrm{BiFeO}_{3}$ were found to be more conductive than the domains (Seidel et al., 2009), while those of $\mathrm{YMnO}_{3}$ were found to be more insulating (Choi et al., 2010a). Multiferroic $\mathrm{YMnO}_{3}$, a socalled improper ferroelectric multiferroic, in which ferroelectricity is induced by structural trimerization coexisting with magnetism, domain walls are found to be insulating (Choi et al., 2010). The increase of the $\mathrm{Y}-\mathrm{O}$ bond distance at domain walls may be responsible for the reduction of local conduction. The observed conduction suppression at domain walls at high voltages (still much less than the electric coercivity) is in striking contrast with what was reported on $\mathrm{BiFeO}_{3}$.

A useful clue for interpreting these results is perhaps the analysis of the paraphase. Whereas the high-temperature, high-symmetry phase of $\mathrm{BiFeO}_{3}$ is more conducting than the ferroelectric phase (Palai et al., 2008), the converse is true for $\mathrm{YMnO}_{3}$ (Choi et al., 2010). This illustrates an important point: In some respects the internal structure of the walls can be considered to be in the paraelectric state; by way of trivial example, the $180^{\circ}$ domain walls of a ferroic are nonpolar, just like its paraphase. The examples of $\mathrm{BiFeO}_{3}$ and $\mathrm{YMnO}_{3}$ suggest that the paraphaselike behavior can be applied to domain wall properties other than just the polarization: The insulating paraelectric state of $\mathrm{YMnO}_{3}$ is consistent with the insulating nature of its domain walls, and conversely the conducting state of the paraphase of $\mathrm{BiFeO}_{3}$ is consistent with its domain wall conductivity. Nevertheless, in the conductivity of the $\mathrm{BiFeO}_{3}$ walls at least there are several other considerations: octahedral rotations, electrostatic steps arising from rigid rotation of the polar vector, and increased carrier density at the wall are all thought to play a role in the domain wall conductivity of $\mathrm{BiFeO}_{3}$ and potentially also of other perovskites. A more detailed discussion of these factors is provided in Sec. V.F.1 .
The resistive behavior of purely magnetic domain walls has also been studied. The domain walls of metallic ferromagnets were found to be more resistive than the domains due to spin scattering (Viret et al., 2000; Danneau et al., 2002). On the other hand, the domain walls of manganites (which are ferromagnetic and ferroelastic) have been predicted to be more conducting than the domains, due to strain coupling: The Jahn Teller distortion is smaller and the octahedral rotation angle is straighter inside the domain wall than outside (Salafranca, Yu, and Dagotto, 2010), leading to increased orbital overlap and thus bigger bandwidth. The same interplay between octahedral rotation straightening and increased conductivity has been postulated for the domain walls of bismuth ferrite (Catalan and Scott, 2009), and the straightening of the octahedral rotation angle inside the walls of this material has been confirmed by electron microscopy (Borisevich et al., 2010). To complete the picture, it should be mentioned that enhanced domain wall conductivity has also been observed in standard ferroelectrics such as $\mathrm{Pb}(\mathrm{Zr}, \mathrm{Ti}) \mathrm{O}_{3}$ (Guyonnet et al., 2011), suggesting that this may be a more general property than previously thought.

The challenge is now to make a resistive switching device based on domain walls. Two approaches may be pursued here. One was suggested by Lee and Salje (2005), who observed that the percolation of a zigzag configuration of ferroelastic walls between the two surfaces of a crystal could be controlled by bending. The other approach pursued is selective doping. The experimental study of domain wall conductivity and the electronic devices that can be made using wall properties will be the subject of the following sections.

\section{EXPERIMENTAL METHODS FOR THE INVESTIGATION OF DOMAIN WALLS}

A variety of structural and near-field probes are available to probe both the macroscopic and microscopic details of domain walls. Atomic-scale imaging of the domain wall structure is now possible with transmission electron microscopy, but of particular emphasis in this review is scanning probe (scanning tunneling microscopy, atomic force microscopy, conducting AFM (c-AFM), and the related piezoforce microsocopy) techniques, which allow the probing of actual functional properties of the domain walls. Readers interested in the details of all of these structural probe techniques are referred to several reviews on this subject; here we give an overview of the information pertinent to domain walls.

\section{A. High-resolution electron microscopy and spectroscopy}

Among the methods available for the investigation of domain walls is high-resolution electron microscopy (Goo et al., 1981; Bursill, Peng, and Feng, 1983; Bursill and Lin, 1986; Stemmer et al., 1995; Hytch, 1998; Lichte, 2002; Jia, 2003). This method allows direct visualization of the lattice distortion across the domain wall by measuring the continuous deviation of a set of planes with respect to the undistorted lattice (exit-wave reconstruction) (Foeth et al., 1999). Current, state-of-the-art techniques permit atomic-scale resolution at $0.5 \AA$ through aberration-corrected imaging 

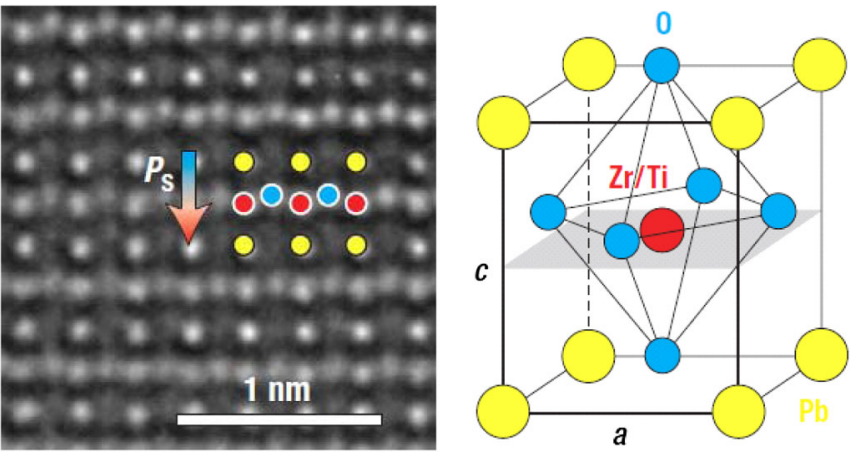

FIG. 27 (color online). Atomic-scale TEM image of the electric dipoles formed by the relative displacements of the $\mathrm{Zr} / \mathrm{Ti}$ cation columns and the $\mathrm{O}$ anion columns in $\mathrm{PbZr}_{0.2} \mathrm{Ti}_{0.8} \mathrm{O}_{3}$. Adapted from Jia et al., 2008.

(Figs. 27 and 28). The exit-wave reconstruction approach eliminates the effects of objective-lens spherical aberrations, and images can be directly interpreted in terms of the projection of the atomic columns (Allen et al., 2004). Weakbeam transmission electron microscopy has been used for a quantitative analysis of the thickness fringes that appear on weak-beam images of inclined domain walls. By fitting simulated fringe profiles to experimental ones, it is possible to extract the thickness of the domain walls in a quantitative way. Regarding high-resolution transmission electron microscopy (HRTEM) images of domain walls, it has to be taken into account that the samples in these kinds of experiments are thin (typically a few nanometers) so that surface pinning of the domain walls could play an important role (Meyer and Vanderbilt, 2002). The atomic displacements across a typical wall are on the order of $0.02 \mathrm{~nm}$, which still makes direct imaging and interpretation a challenge (Gopalan, Dierolf, and Scrymgeour, 2007). HRTEM also offers the possibility of imaging the local polarization dipoles at atomic resolution, thus quantitatively measuring the local polarization and investigating the domain structure (Jia et al., 2008).

Using the negative spherical-aberration imaging technique in an aberration-corrected transmission electron microscope, large differences in atomic details between charged and uncharged domain walls have been reported, and cation-oxygen dipoles near $180^{\circ}$ domain walls in epitaxial $\mathrm{PbZr}_{0.2} \mathrm{Ti}_{0.8} \mathrm{O}_{3}$ thin films have been resolved on the atomic scale (Jia et al., 2008).
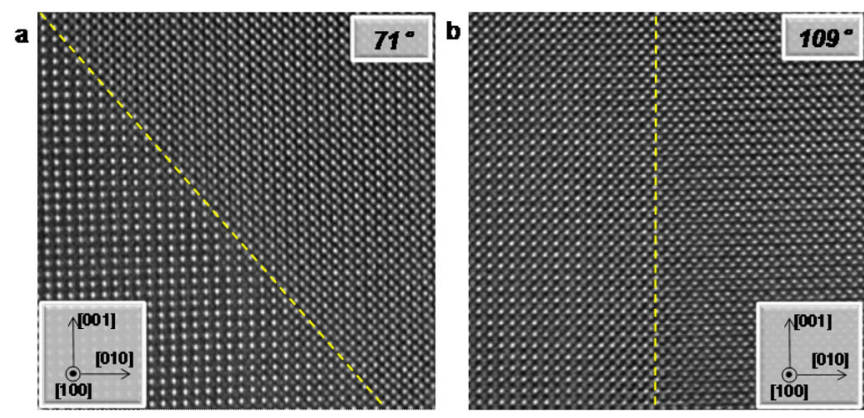

FIG. 28 (color online). (a) $71^{\circ}$ and (b) $109^{\circ}$ domain walls in bismuth ferrite.
Elemental and electronic structure analysis by electronenergy-loss spectroscopy has also been applied to the study of domain walls (Jia and Urban, 2004; Urban et al., 2008). Using high-resolution imaging in an aberration-corrected TEM, the concentration of oxygen in $\mathrm{BaTiO}_{3}$ twin boundaries was measured at atomic resolution. These measurements provide quantitative evidence for a substantial reduction of the oxygen occupancy, i.e., the presence of oxygen vacancies at the boundaries. It was also found that the modified $\mathrm{Ti}_{2} \mathrm{O}_{9}$ group unit formed reduces the grain boundary energy and provides a way of accommodating oxygen vacancies occurring in oxygen-deficient materials. This type of atomically resolved measurement technique offers the potential to study oxide materials in which the electronic properties sensitively depend on the local oxygen content (important in view of current work on $\mathrm{LaAlO}_{3} / \mathrm{SrTiO}_{3}$ superconducting interfaces). The attraction between domain walls and vacancies is further discussed in Sec. V.

\section{B. Scanning probe microscopy}

Atomic force microscopy and its variations (e.g., c-AFM, PFM) are well suited for direct writing ("ferroelectric lithography") and characterization of prototype ferroelectric structures (Fig. 29), including domain walls (Eng, 1999). These methods provide the tools to get information about local mechanisms of twin-wall broadening that cannot be obtained by existing experimental methods (Shilo, Ravichandran, and Bhattacharya, 2004). With conductive AFM (c-AFM) one can artificially modify the domain structure as a function of pulse width and amplitude (Tybell et al., 2002). PFM is also under continuous development and is currently undergoing a shift of focus from imaging static domains to (i) dynamic characterization of the switching process (with developments such as stroboscopic PFM and PFM spectroscopy) and (ii) the structure of domain walls (Gruverman et al., 2005; Jungk, Hoffmann, and Soergel, 2006; Rodriguez et al., 2007a, 2007b; Morozovska et al., 2008; Kalinin et al., 2010) (see also Fig. 30).

Let us take a closer look at the relationship between domain walls and the effect of electric fields on ferroelectrics. When the applied field is higher than the coercive field, the walls will move; however, the threshold field at which preexisting domain walls begin to move can be much lower than
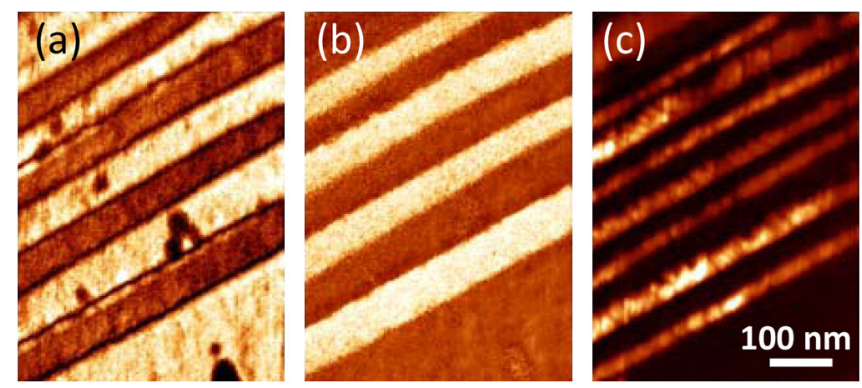

FIG. 29 (color online). (a) PFM amplitude and (b) PFM phase images of a BFO sample with $109^{\circ}$ stripe domains; (c) simultaneously acquired c-AFM image of the same area showing that each $109^{\circ}$ domain wall is electrically conductive. From Seidel et al., 2010. 


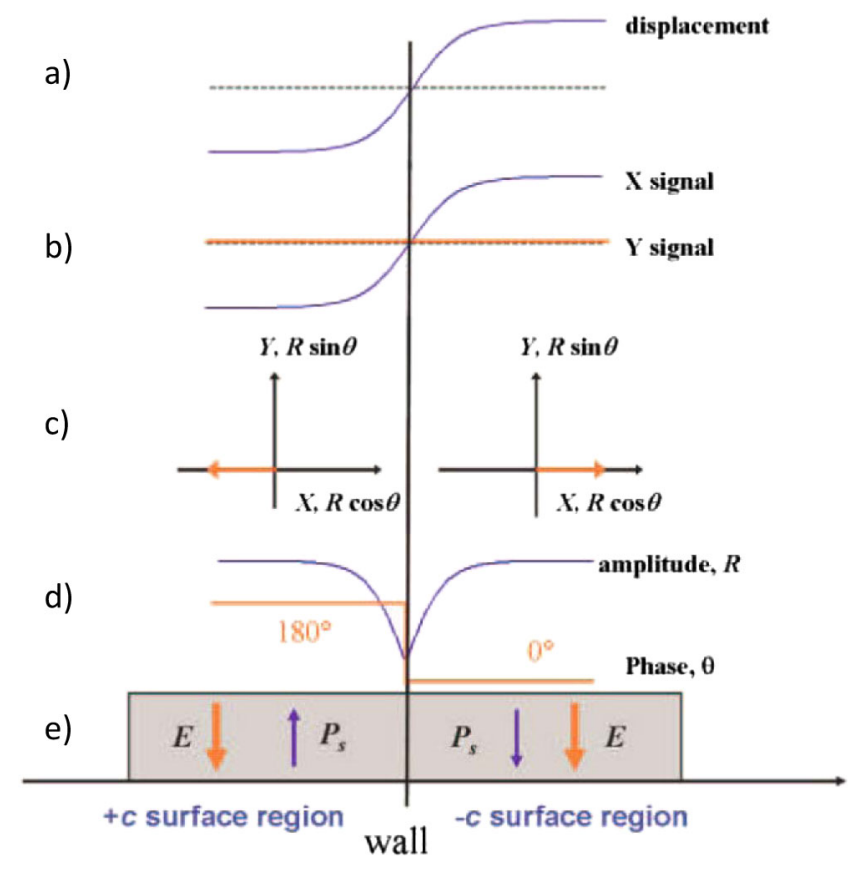

FIG. 30 (color online). Schematic piezoresponse across a single $180^{\circ}$ domain wall in lithium niobate crystal. (a) The surface displacement (solid line) due to the electric field across the domain wall displayed in (e). The dotted line is the original surface plane. (b) The piezoresponse, both $X$ and $Y$ signals, across the domain wall. $X$ is the product of amplitude $(R)$ and the sine of the phase, $q$, and $Y$ is the product of amplitude and cosine of the phase. (c) The piezoresponse, both $X$ signal and $Y$ signals, on both $+c$ and $-c$ surfaces plotted in a vectorial $X Y$ plane. (d) The amplitude and phase of the piezoresponse across the domain wall. (e) Schematic domain structure and electrical field. From Tian, 2006.

the coercive field required for nucleation (Gopalan, Dierolf, and Scrymgeour, 2007; Choudhury et al., 2008). From preliminary theory and experiments, Gopalan, Dierolf, and Scrymgeour (2007) argued that the thickness of the domain wall is different when an external electric field is applied because of changes in its Landau energy potential. This prediction is also supported by the calculations of Rao and Yu (2007) and Hlinka, Ondrejkovic, and Marton (2009). For $\mathrm{PbTiO}_{3}$, the effect of an applied electric field leads to an increase in the wall thickness of 2 to 4 times and for $\mathrm{LiNbO}_{3}$ the thickness increases up to $10 \%-30 \%$, but this phenomenon is expected to be general for all compositions. At the same time, a thicker (or diffuse) domain wall has a lower threshold field (Choudhury et al., 2008) for lateral movement, as it is not narrow enough to drag against the washboardlike Peierls potential. This effect was used to explain low threshold fields for domain reversal in ferroelectrics. However, the same authors predict that an increase in wall thickness at surfaces will be of influence only when the crystal thickness is $1-10 \mathrm{~nm}$ and that, in general, the region with the lowest wall thickness will dominate the threshold field for the motion of the entire wall.

Analysis of both complete area images and individual linescan profiles provides essential information about local mechanisms of twin-wall broadening, which cannot be obtained by other experimental methods (Shilo, Ravichandran, and Bhattacharya, 2004). Surface topography measured using atomic force microscopy is compared with candidate displacement fields, and this allows for the determination of the twin-wall thickness and other structural features. Closedform analytical expressions for vertical and lateral PFM profiles of a single ferroelectric domain wall for the conical and disk models of the tip, beyond point charge and sphere approximations have been investigated (Morozovska et al., 2008). Here the analysis takes into account the finite intrinsic width of the domain wall and dielectric anisotropy of the material. The analytical expressions provide insight into the mechanisms of PFM image formation and can be used for a quantitative analysis of the PFM domain wall profiles.

Ferroelectric thin films typically contain various structural defects such as cationic and/or anionic point defects, dislocations, and grain boundaries. Since the electric and stress fields around such defects in a ferroelectric thin film are likely to be inhomogeneous, it is expected that the switching behavior near a structural defect will be different from the one found in a single-domain state. The role of a single ferroelastic twin boundary has been studied in tetragonal $\mathrm{PbZr}_{0.2} \mathrm{Ti}_{0.8} \mathrm{O}_{3}$ ferroelectric thin film (Choudhury et al., 2008). It was shown that the potential required to nucleate a $180^{\circ}$ domain is lower near ferroelastic twin walls $(\sim 1.6 \mathrm{~V})$ compared with $\sim 2.6 \mathrm{~V}$ away from the twin walls.

A recently increased interest in combined PFM and conductivity measurements arises from both nonvolatile memory application perspective and a potential for electroresitive memory devices (Yang et al., 2009). The work of Gruverman, Isobe, and Tanaka (2001) explored the interplay of domain dynamics and conductivity at interfaces in thin ferroelectric films. The combination of local electromechanical and conductivity measurements revealed a connection between local current and pinning at bicrystal grain boundaries in bismuth ferrite (Rodriguez et al., 2008). Electroresistance in ferroelectric structures was recently reviewed by Watanabe (2007). The presence of extended defects and oxygen vacancy accumulation has been shown to influence transport mechanisms at domain walls (Seidel et al., 2010). Recently, direct probing of polarizationcontrolled tunneling into a ferroelectric surface was shown (Garcia et al., 2009; Maksymovych et al., 2009). Scanningnear-field optical microscopy has been used to observe pinning and bowing of a single $180^{\circ}$ ferroelectric domain wall under a uniform applied electric field (Yang, 1999; Kim et al., 2005).

Typically the imaging resolution in PFM is about $5-30 \mathrm{~nm}$. The achievable resolution is ultimately limited by the tipsample contact area, which is nominally determined by the radius of the tip apex. There are additional mechanisms for resolution broadening such as electrostatic interactions and the formation of a liquid neck under ambient conditions in the tip-surface junction. The PFM amplitude typically provides information on the magnitude of the local electromechanical coupling under the tip, and the PFM phase gives information about the ferroelectric domain orientation.

Scanning tunneling spectroscopy can be used to directly probe the superconducting order parameter at nanometer length scales. Scanning tunneling microscopy (STM) and spectroscopy (STS) have been used to investigate the electronic structure of ferroelastic twin walls in $\mathrm{YBa}_{2} \mathrm{Cu}_{3} \mathrm{O}_{7-\delta}$ 
(YBCO) (Maggio-Aprile et al., 1997). Twin boundaries play an important role in pinning the vortices and thereby enhancing the currents that YBCO can support while remaining superconducting. An unexpectedly large pinning strength for perpendicular vortex flux across such boundaries was found, which implies that the critical current at the boundary approaches the theoretical "depairing" limit.

In the case of insulators, STM and STS are by definition a lot more difficult to implement, primarily because a reliable tunneling current cannot be used to establish proximal contact. The emergence of ferroelectrics with smaller band gaps and the possibility of conduction at domain walls (see later) has stimulated renewed interest in exploring STM as a probe of the local electronic structure. The emergence of combined AFM and STM or SEM (scanning electron microscopy) and STM systems should be a boon in terms of exploring the electronic properties of domain walls in such insulating materials. Research using such combined tools is in its infancy (Wiessner et al., 1997; Yang et al., 2005; García, Huey, and Blendell, 2006; Chiu et al., 2011).

\section{X-ray diffraction and imaging}

Diffuse x-ray scattering can also be used to investigate the structure of domains and domain walls in densely twinned ferroelastic crystals (Bruce, 1981; Andrews and Cowley, 1986; Locherer, Chrosch, and Salje, 1998). The scattering is characterized by strong, well-defined Bragg peaks, with a diffuse streak between these arising from the domain walls (see Fig. 31). The streak typically is several orders of magnitude lower in intensity. Comparison with an analytical model for the scattering allows one to extract the effective domain wall width on the unit-cell level (Locherer, Chrosch, and Salje, 1998). Critical fluctuations and domain walls of, for example, $\mathrm{KH}_{2} \mathrm{PO}_{4}(\mathrm{KDP})$ and $\mathrm{KD}_{2} \mathrm{PO}_{4}(\mathrm{DKDP})$ were investigated (Andrews and Cowley, 1986). The intensity of the



FIG. 31 (color online). X-ray intensity profile of the $(400) /(040)$ peak along (110) in a $\mathrm{WO}_{3}$ crystal. The dashed line shows a Gaussian fit to the contribution from the domain walls. The bold solid line is the overall fit. Figure courtesy of Ekhard Salje, adapted from Locherer, Chrosch, and Salje, 1998. critical scattering near two different reciprocal lattice points was determined and used to find the atomic displacements in the ferroelectric fluctuations. The x-ray scattering from the domain walls was observed below $T_{c}$ and enabled measurements to be made of the width of the domain walls and the atomic displacements in the walls. The width of the domain walls was shown to increase with temperature toward $T_{c}$.

Synchrotron $\mathrm{x}$-ray sources have also been used for direct imaging of strain near ferroelectric $180^{\circ}$ domain walls in congruent $\mathrm{LiNbO}_{3}$ and $\mathrm{LiTaO}_{3}$ crystals and in $\mathrm{BaTiO}_{3}$ crystals (Kim, 2000; Rogan, 2003; Jach, 2004). Direct evidence for wide regions of strain on length scales of many micrometers associated with $180^{\circ}$ domain walls in congruent $\mathrm{LiNbO}_{3}$ and $\mathrm{LiTaO}_{3}$ crystals was found. The observed strain contrast in symmetric high-resolution diffraction images in Bragg geometry arises in part from curvature in the basal planes across a domain wall as well as from lateral variation in the lattice spacing of the basal planes extending across a wall. In $\mathrm{BaTiO}_{3}$ local triaxial strain fields around $90^{\circ}$ domains were found. Specifically, residual strain maps in a region surrounding an isolated, approximately $40-\mu \mathrm{m}$-wide, $90^{\circ}$ domain were obtained, revealing significant residual strains.

\section{Optical characterization}

Ferroelectrics offer the possibility of engineering their domain structure down to the nanometer regime and therefore allow for interesting optical functionality such as mode shaping and frequency conversion, as well as the integration into compact optical devices (Chen et al., 2001; Kurz, Xie, and Fejer, 2002; Scrymgeour et al., 2002). Ferroelectric crystals in general are anisotropic and show birefringence. Regions with different orientations of the polar axis are, for example, easily differentiated by polarization microscopy (Tarrach et al., 2001). Because of the symmetry of the optical indicatrix, regions of opposite polarization cannot be distinguished by linear optics. Nonlinear effects, however, such as secondharmonic generation (Dolino, 1973) or the electro-optic effect (Hubert and Levy, 1997; Otto et al., 2004) revealed the eccentricity of the crystal. Regarding materials, lithium niobate $\left(\mathrm{LiNbO}_{3}\right)$ and lithium tantalate $\left(\mathrm{LiTaO}_{3}\right)$ emerged as key technological materials for photonic applications (se Fig. 32). High quality of crystal growth, optical transparency over a

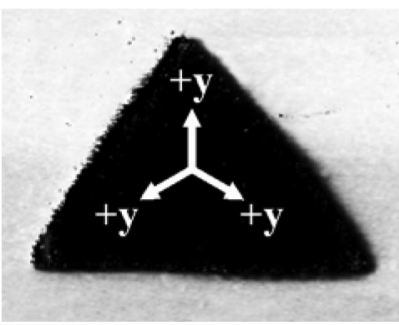

Congruent $\mathrm{LiTaO}_{3}$

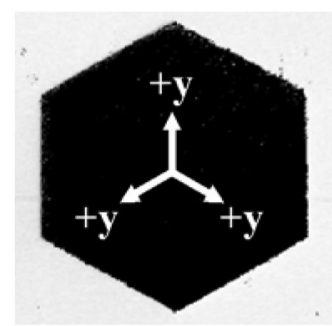

Congruent $\mathrm{LiNbO}_{3}$ Stoichiometric $\mathrm{LiNbO}_{3}$ Stoichiometric $\mathrm{LiTaO}_{3}$
FIG. 32. Piezoelectric force microscopy phase contrast images of domain shapes in $\mathrm{LiNbO}_{3}$ and $\mathrm{LiTaO}_{3}$. From Scrymgeour et al., 2005. 
wide frequency range ( $240 \mathrm{~nm}$ to $4.5 \mu \mathrm{m}$ wavelength), and their large electro-optic and nonlinear optical coefficients are the main advantages of these materials. Emerging fields of optical communication, optical data storage, optical displays, biomedical device applications, and sensors all rely heavily on such ferroelectric materials as a versatile solid-state photonic platform. The process of domain control is difficult and has received tremendous attention over the past years. The central focus of work has been set on developing a fundamental understanding of shaping and controlling domain walls in ferroelectrics, specifically in lithium niobate and lithium tantalate, for photonic applications.

An understanding of the domain wall phenomena has been approached at the macroscale and the nanoscale. Different electric-field poling techniques have been developed and used to create domain shapes of arbitrary and controlled orientation. A theoretical framework based on the Landau-Devonshire theory is typically used to determine the preferred domain wall shapes in these materials (Lines and Glass, 2004). Differences in the poling characteristics and domain wall shapes between the materials as well as differences in material composition have been identified to be related to nonstoichiometric defects in these crystals. (Shur, 2006).

\section{APPLICATIONS OF DOMAINS AND DOMAIN WALLS}

When applying an external field to a material with domains, the walls will move so as to expand those domains that are energetically favored by the field and contract those that are not. Having a large density of mobile domain walls facilitates this change in domain populations and can therefore dramatically enhance the susceptibility of any ferroic, be it magnetic susceptibility, elastic compliance, dielectric constant or piezoelectric coefficient. The contribution of domain walls to the susceptibility of ferroelectrics was first studied in Prague more than four decades ago (Fouskova, 1965; Fousek and Janoušek, 1966), and presently there exist good articles and reviews about the dynamic domain wall contribution to susceptibility and piezoelectricity (Zhang et al., 1994; Bassiri-Gharb et al., 2007) so we do not dwell on this topic here. Instead, we focus on the applications of static ferroelectric domain configurations (chiefly, electro-optical devices) on one hand, and on the newer concept of devices exploiting domain wall shift (the so-called racetrack memories).

\section{A. Periodically poled ferroelectrics}

Prior to the recent flurry of activity on domain engineering, the primary device application requiring control and manipulation of ferroelectric domains involved periodic poling of ferroelectrics. This application is for nonlinear optics, such as second-harmonic generation: The efficiency of the wavelength conversion is increased by having periodic antiparallel domains, with the maximum theoretical efficiency being achieved when the wavelength of the pump laser matches the full repeat length of a pair of $+P$ and $-P$ domains, as first pointed out by Bloembergen in his Nobel prize-winning work (Armstrong et al., 1962).

The production of highly efficient nonlinear electro-optic devices via the technique of periodically poling ferroelectric crystals (quasiphase matching) emphasized devices made from lithium niobate $\left(\mathrm{LiNbO}_{3}\right)$ and lithium tantalate $\left(\mathrm{LiTaO}_{3}\right)$, both congruent and stoichiometric, KTP $\left(\mathrm{KTiOPO}_{4}\right)$, and tungsten bronzes of the barium sodium niobate family. Generally speaking, these have been successful commercial devices, but a few problems remain that prevent optimization of real products. First, the domain widths are sometimes not stable with time; second, there is a particular problem in achieving narrow (submicrometer) widths. In this section we examine some real device parameters and suggest that the crystal (or film) thicknesses have not been optimized in a way that is compatible with the domain widths, connected through the Landau-Lifshitz-Kittel law (see Table I).

Since the original report of efficient nonlinear optics from phase-matched periodically poled ferroelectrics by Armstrong et al. (1962) there have been numerous developments and commercial production of such devices, starting about two decades ago, first in Japan (Yamada et al., 1993), and then in the USA (Myers et al., 1995). From the early 1990s interest was perhaps evenly divided between KTP $\left(\mathrm{KTiOPO}_{4}\right)$ (Chen and Risk, 1994; Karlsson, 1997; Reid,

TABLE I. Some device parameters for periodically poled ferroelectrics. Wall thicknesses $t$ measured using PFM or optical methods overestimate the true crystallographic wall thicknesses, as pointed out by Jungk, Hoffmann, and Soergel (2007). The quoted wall thickness for KTP is therefore likely to be too high.

\begin{tabular}{|c|c|c|c|c|}
\hline $\begin{array}{l}\text { Sample thickness } d \\
\qquad(\mathrm{~mm})\end{array}$ & $\begin{array}{l}\text { Domain width } w \\
(\mathrm{~nm})\end{array}$ & $\begin{array}{l}\text { Wall thickness } t \\
(\mathrm{~nm})\end{array}$ & $\begin{array}{l}\text { Domain depth } d^{\prime} \\
(\mathrm{nm})\end{array}$ & Reference \\
\hline \multicolumn{5}{|l|}{$\overline{\mathrm{KTiOPO}_{4}}$} \\
\hline $0.5 \mathrm{~mm}$ & $283-360 \mathrm{~nm}$ & $20-80$ ( $a$ face $)$ & $\approx 100 \mathrm{~nm}$ & $\begin{array}{l}\text { Wittborn et al. (2002); Canalias, } \\
\text { Pasiskevicius, and Laurell (2005); } \\
\text { Laurell and Canalias (2009) }\end{array}$ \\
\hline $0.5 \mathrm{~mm}$ & & 65 ( $b$ face) & & $\begin{array}{l}\text { Canalias, Pasiskevicius, and } \\
\text { Laurell (2006); Canalias et al. (2006) }\end{array}$ \\
\hline \multicolumn{5}{|l|}{$\mathrm{LiNbO}_{3}$} \\
\hline $1 \mathrm{~mm}$ & $150 \mathrm{~nm}-6 \mu \mathrm{m}$ & $<3 \mathrm{~nm}$ & $<100 \mathrm{~nm}$ & $\begin{array}{l}\text { Shur et al. (2000); Rosenman et al. } \\
\text { (2003a, 2003b); Grilli et al. (2005); } \\
\text { Jungk, Hoffmann, and Soergel (2007) }\end{array}$ \\
\hline $\begin{array}{l}\mathrm{LiTaO}_{3} \\
0.15\end{array}$ & $875 \mathrm{~nm}$ & & & Mizuuchi, Yamamoto, and Kato (1997) \\
\hline
\end{tabular}


1997; Wang, 1998; Rotermund, 1999) and lithium niobate (Hu, Thomas, and Webjörn, 1996; Galvanauskas, 1997; Penman, 1998). This mix of materials continued in more recent studies (Rosenman et al., 2003a, 2003b; Tiihonen, Pasiskevicius, and Laurell, 2006; Canalias, Pasiskevicius, and Laurell, 2006; Canalias et al., 2006; Lagatsky et al., 2007; Henriksson et al., 2006; Hirohashi et al., 2007), augmented by results on the tungsten bronzes of the $\mathrm{Ba}_{2} \mathrm{NaNb} \mathrm{O}_{15}$ family (Jaque et al., 2006). Note that, as pulled from the congruent melt, $\mathrm{LiNbO}_{3}$ is not stoichiometric; the spectroscopic differences between congruent and stoichiometric $\mathrm{LiNbO}_{3}$ were first illuminated by Okamoto, Wang, and Scott (1985). We also note parenthetically that $\mathrm{LiNbO}_{3} / \mathrm{LiTaO}_{3}$ and $\mathrm{KTiOPO}_{4}$ are both nonferroelastic ferroelectrics since their crystal classes do not change at their ferroelectric phase transitions (rhombohedral-rhombohedral and orthorhombic-orthorhombic, respectively). This means that only $180^{\circ}$ domains are present, which simplifies switching dynamics, and it implies that there is no hysteretic stress during switching, which minimizes energy cost in poling.

\section{Application of Kittel's law to electro-optic domain engineering}

As discussed in Sec. II, the domain width $w$ is proportional to the square root of the crystal (or film) thickness $d$ (see Fig. 1). The proportionality constant is material dependent and not easily evaluated, and this was simplified by the group of Luk' yanchuk (De Guerville et al., 2005) and by some of us (Catalan et al., 2007a): By dividing the Landau-LifshitzKittel formula by the domain wall thickness $\delta$, a dimensionless constant results [Eq. (2)]. Our basic hypothesis, already advanced by Catalan et al. (2007a), is that periodically poled electro-optic devices often have domain periodicities and domain walls that are unstable with time because they are fabricated at thicknesses and widths that do not satisfy the Kittel equation [Eq. (2)]. We consider how to improve and optimize this situation.

Note three things in Eq. (2): First, the domain wall thickness $\delta$ is assumed to be an intrinsic constant, whereas, in fact, $\delta$ can be manipulated experimentally to optimize stability. Secondly, $d$ is not necessarily the thickness of the crystal, but the depth of the domains, which can be much less than the total film thickness. And, finally, the material-specific parameter in Eq. (2) is the square root of the ratio of in-plane and out-of-plane electric susceptibilities. As shown, this ratio is different for the three electro-optic materials recently explored for periodically poled devices. The devices are typically fabricated on specimens that are 0.15 to $1.0 \mathrm{~mm}$ in thickness. The typical domain widths that one strives for (in order to match visible or near-visible wavelengths) are $100-900 \mathrm{~nm}$, and the typical wall thicknesses are $10 \mathrm{~nm}$ or less. These numbers do not satisfy the Kittel law; in particular, for a domain wall thickness of $10 \mathrm{~nm}$, a 500-nm domain width would be thermodynamically stable in $\mathrm{LiNbO}_{3}$ or $\mathrm{KTiOPO}_{4}$ only for a much thinner specimen of $\approx 10 \mu \mathrm{m}$, significantly less than the actual $1 \mathrm{~mm}$.

It is not easy to circumvent thermodynamics or to fool Mother Nature. If one constrains the domain widths to be smaller than the equilibrium Kittel value via spatially abrupt applied fields, thermodynamics "retaliates" by making the

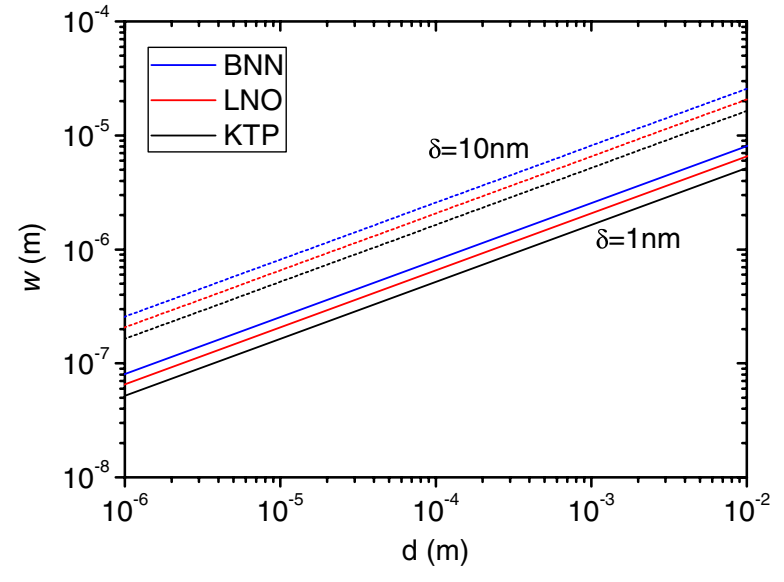

FIG. 33 (color online). Domain size as a function of thickness for three uniaxial ferroelectrics commonly used in electro-optical applications. The continuous lines are calculated assuming domain wall thickness of $1 \mathrm{~nm}$, while the dashed lines are for a wall thickness of $10 \mathrm{~nm}$.

stable domains not penetrate through the sample from anode to cathode of the applied poling voltage, but instead only partially to a few micrometers in depth (Batchko et al., 1999).

For $\mathrm{Ba}_{2} \mathrm{NaNb}_{5} \mathrm{O}_{15}$ the out-of-plane (polar-axis) dielectric constant is 32 . The in-plane is biaxial but nearly isotropic at 222 ( $x$ axis) and 227 ( $y$ axis) (Warner, Coquin, and Frank, 1969). So the square root of the ratio is 2.65. Hence for excitation with 1.064- $\mu \mathrm{m} \mathrm{Nd:YAG} \mathrm{(yttrium} \mathrm{aluminum} \mathrm{gar-}$ net) (doubling to the green at $532 \mathrm{~nm}$ ), and remembering that two ferroelectric stripes make up one full wavelength, we calculate the optimum thickness $d$ from Eq. (2): $w^{2} /(d \delta)=$ $2.455 \times 2.65=5.5$. For $\delta$ of $1.0 \mathrm{~nm}$ (more about this choice below), this yields an optimum $d=180 \mu \mathrm{m}$. For $\mathrm{LiNbO}_{3}$ (congruent) the out-of-plane dielectric constant is 27.9 and the in-plane one is 85.2 (Smith and Welsh, 1971). Therefore the ratio is 3.05 and $w^{2} /(d \delta)=2.455 \times 1.75=4.3$. For $\mathrm{KTP}$, the dielectric constant is unusually low (average 13.0); the in-plane values are 11.3 and 11.9 (average 11.6), and the out-of-plane one is frequency dependent but $\approx 17.5$ at low frequencies and 15.4 at high frequencies (Bierlein and van Herzeele, 1989; Noda et al., 2000). Using the high-frequency value, this gives a susceptibility ratio of 1.2 , for which the square root is 1.1 . Hence, $w^{2} /(d \delta)=$ $2.455 \times 1.1=2.7$. For the $1-n m$ domain wall thickness given above as an example, this requires a crystal thickness two and one-half times as great as that for $\mathrm{LiNbO}_{3}$, approximately $0.45 \mathrm{~mm}$. These three materials thus require different thicknesses for optimum phase matching at $1 \mu \mathrm{m}$, as shown in Fig. 33. As can be seen, the different dielectric anisotropy of the three materials has a relatively small impact on the optimum domain size, due to the fact that the crystal anisotropy is inside a square root of a square root. A much bigger variation of optimum domain size can be obtained by tuning instead the domain wall thickness, as discussed in the next section.

\section{Manipulation of wall thickness}

The wall thickness parameter $\delta$ is not an intrinsic constant. It can be increased by an order of magnitude by impurity 
doping and it can also be increased via application of an electric poling field orthogonal to the polar axis.

\section{a. Doping}

We see in Reznik et al. (1985) that impurity doping can greatly increase the domain wall thickness in ferroelectrics. This will decrease the stability thickness $d$ for a given wavelength, or alternatively permit longer-wavelength electro-optic devices for a fixed film thickness. Similarly, wall thicknesses in congruent lithium niobate are about 10 times thicker than in stoichiometric specimens. For many years only $\mathrm{LiNbO}_{3}$ grown from a congruent melt was available for study. These crystals have $1 \%$ (or $10^{21} \mathrm{~cm}^{-3}$ ) defects. The spectroscopic difference between congruent and stoichiometric $\mathrm{LiNbO}_{3}$ was first shown by Okamoto, Wang, and Scott (1985) and Chowdhury (1978). Recent periodically poled $\mathrm{LiNbO}_{3}$ devices favor stoichiometric samples because their domain walls are more stable. See, for example, Chu et al. (2008).

\section{b. Photovoltaic tensor and off-axis poling}

The theoretical model of Rao and Wang (2007) implies that off-axis poling can significantly widen wall thicknesses. This brings us into a more general discussion of photovoltaic tensors. Over the years, perhaps misled by the standard text by Lines and Glass (2004), which implies that photovoltaic response in $\mathrm{LiNbO}_{3}$ is along the polar $z$ axis, many scientists failed to recognize that the photovoltaic tensor is neither diagonal nor second rank, despite the correct theory of Chen (1968, 1969). As a result, large voltages and fields can arise perpendicular to the polar axis when illuminated. In a $1-W$ beam at $514.5 \mathrm{~nm}$ wavelength, focused to $\approx 50 \mu \mathrm{m}$ diameter, lithium niobate exhibits a field of approximately $40 \mathrm{kV} / \mathrm{cm}$ in the $x y$ plane, due to the $\beta_{15}$ photovoltaic tensor component (Odulov, 1982, Anikiev et al., 1985; Reznik et al., 1985; Chaib, Otto, and Eng, 2003) (note that we used the reduced notation, the photovoltaic tensor is third rank).
These off-axis photovoltages can be mitigated via application of a thermal gradient, which causes charge diffusion to mitigate the photovoltaic effect via the Seebeck effect (Kostritskii et al., 2007, 2008). Hence it would be useful to more carefully examine the effects of off-axis poling and of photovoltage normal to the polar axis. In particular, in the presence of high-intensity laser light the symmetry of $\mathrm{LiNbO}_{3}$ is actually lowered; the threefold symmetry axis is lost.

\section{B. Domains and electro-optic response of $\mathrm{LiNbO}_{3}$}

In assessing the microscopic dynamics of domains in poled lithium niobate, it is useful to point out that the local electric field is not necessarily along the polar axis, and that for electro-optic devices, in the presence of light there is a strong electric polarization induced perpendicular to this threefold $c$ axis. This was first established by Chen (1969), and later evaluated quantitatively by Anikiev et al. (1985) who found an orthogonal electric field of $\approx 40 \mathrm{kV} / \mathrm{cm}$ in the presence of moderately focused $0.5 \mathrm{~W}$ power at $514.5 \mathrm{~nm}$ from an argon laser. Most recently this result has been confirmed by Chaib, Otto, and Eng (2003). We emphasized this point here because it is contrary to the claims in the textbook by Lines and Glass (2004) (its first edition was written two years before Chen's work). It can significantly influence domain widths. All of these effects differ in congruent and stoichiometric $\mathrm{LiNbO}_{3}$, as do the phonon spectra (Scott, 2002) and especially the quasielastic scattering (Chowdhury, 1978; Okamoto, Wang, and Scott, 1985).

\section{Photovoltaic effects at domain walls}

Recently it was reported that an anomalous photovoltaic effect in BFO thin films arises from a unique, new mechanism, namely, structurally driven steps of the electrostatic potential at nanometer-scale domain walls (Yang et al., 2010;
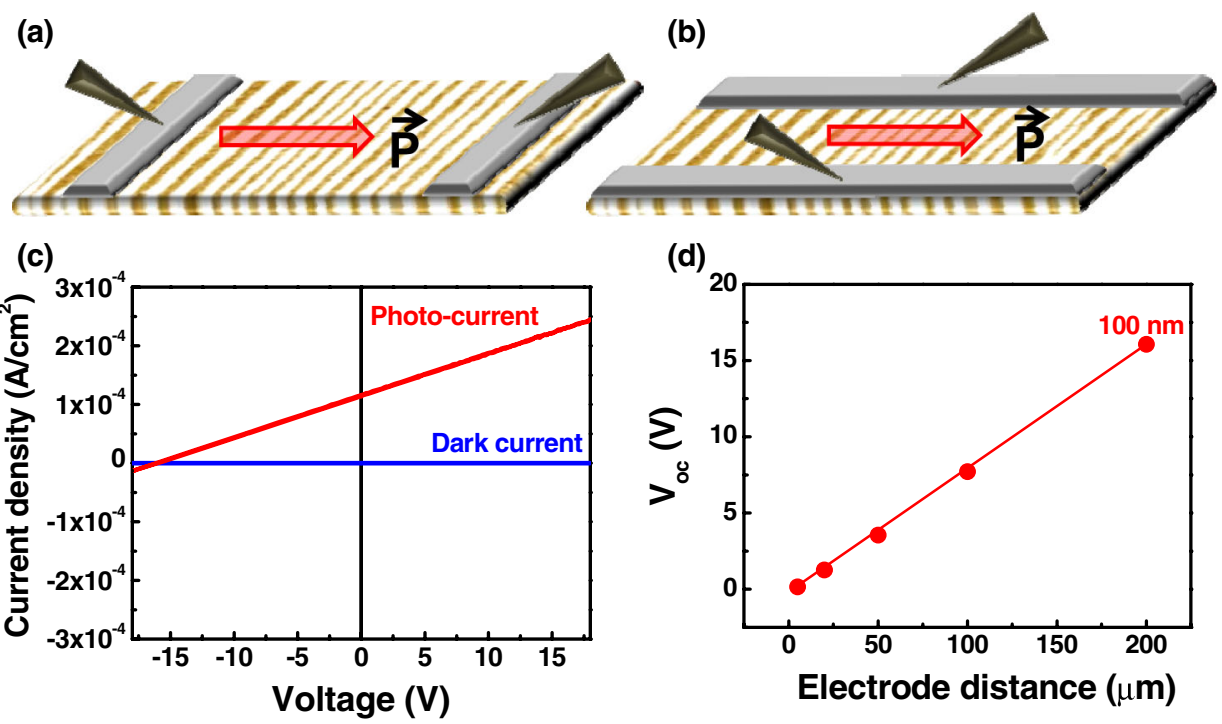

FIG. 34 (color online). Light and dark $I-V$ measurements on ordered arrays of $71^{\circ}$ domain walls in bismuth ferrite showing large open circuit voltages above the band gap of the material. (a), (b) Schematics of the electrode geometry (c) corresponding $I$ - $V$ measurement perpendicular to the domain walls over $200 \mu \mathrm{m}$ distance; (d) linear scaling of open circuit voltage with the number of domain walls. 
Seidel et al., 2011). In conventional solid-state photovoltaics, electron-hole pairs are created by light absorption in a semiconductor and separated by the electric field spanning a micrometer-thick depletion region. The maximum voltage these devices can produce is equal to the semiconductor electronic band gap, although in noncentric systems such as ferroelectrics the photovoltage can be bigger than the band gap (Sturman and Fridkin, 1992). Interestingly, domain walls can give rise to a fundamentally different mechanism for photovoltaic charge separation, which operates over a distance of 1-2 $\mathrm{nm}$ and produces voltages that are significantly higher than the band gap (see Fig. 34). The separation happens at previously unobserved nanoscale steps of the electrostatic potential that naturally occur at ferroelectric domain walls in the complex oxide $\mathrm{BiFeO}_{3}$. Electric-field control over domain structure allows the photovoltaic effect to be reversed in polarity or turned off.

Currently, the overall efficiency of those photovoltaic devices is limited by the conductivity of the bulk bismuth ferrite material. Methods to increase the carrier mobility as well as inducing the spatially periodic potential in an adjacent material with a lower gap than BFO are possible routes to achieve larger current densities under white light illumination, and more generally, they demonstrate what the source of periodic potential and the $P(V)$ current flow can be in different materials. Low-band-gap semiconductors with asymmetric electron and hole mobilities are possible candidates to show such an effect. In addition, photoelectrochemic effects at domain walls are a possible further interesting route, e.g., for applications in water splitting (Kudo and Miseki, 2009).

\section{Switching of domains}

Rather comprehensive reviews of ferroelectric domain switching have been published elsewhere (Shur, Gruverman, and Rumentsev, 1990; Scott, 2000), and so, after a few brief remarks, we concentrate on what is new and particularly pertinent to thin ferroelectric films with high densities (volume fractions) of domain walls or twin boundaries.

At present the best way to monitor domain wall switching is probably via measurement of displacement current $I(t)$ versus time $t$. This gives a rapid rise followed by a roughly Gaussian peak. Assuming that the rise is not current limited from the drive voltage source and that the decay is not limited by the $R C$ time constant, such data are popularly fitted to a model due to Ishibashi and Takagi (1971) and based upon earlier work by Avrami (1939) for the analogous problem of crystal growth. The fitting parameters used involve a characteristic switching time $t(0)$ and, importantly, a dimensionality $D$. One of the important aspects of this theory is that for a given dimensionality there is a precise prediction of the dimensionless ratio $i(m) t(m) / P$, where $i(m)$ is the maximum displacement current density during switching and occurs at time $t(m)$, and $P$ is the spontaneous polarization. In principle the dimension $D$ is an integer, but because of other approximations made in the model, particularly that the domain wall speed $v$ is independent of domain radius $r$ (it actually varies as $1 / r$ ), noninteger values usually result from least-squares fitting to the data. Other approximations are not so important, but Dalton, Jacobs, and Silverman (1971) pointed out that the model fails mathematically for finite dimensions. This model has been used extensively to fit data as functions of field $E$, thickness $d$, temperature $T$, and fatigue cycles $n$. An interesting observation is that domain dimensionality $D$ often decreases from $\approx 3$ to 2 or 1 with fatigue (Araujo et al., 1986).

Of particular interest is what happens in thin films of highly twinned ferroelectrics and ferroelastics. This was first described by Bornarel, Lajzerowicz, and Legrand (1974), who found that in such cases ferroelectric polar domain walls could strongly interact with nearby ferroelastic nonpolar walls, tilting both walls and making the nonpolar walls slightly polar. This was recently demonstrated rather spectacularly in ferroelectric tris-sarcosine calcium chloride by Jones et al. (2011). Of relevance here is also the fact that ferroelectric domain walls are easily pinned by defects and vacancies, so the switching properties and dielectric contribution of the walls can be modified by manipulating the dopant chemistry. Fujii et al. (2010) discussed this in some detail and explicitly showed how defect dipoles are more effective at domain wall pinning than are oxygen vacancies.

\section{E. Domain wall motion: The advantage of magnetic domain wall devices}

The development of prototype devices based upon magnetic domain devices has been pioneered by Cowburn and coworkers (Allwood et al., 2005; Allwood, Xiong, and Cowburn, 2006a, 2006b; Atkinson et al., 2006) as listed and shown schematically in Fig. 1. His devices are suitably small for commercial production (typically $15 F^{2}$, where $F^{2}$ is the square of the feature size $F$ ), and extremely fast. His devices include NOT gates, AND gates, (Zeng et al., 2010) shift registers (O'Brien et al., 2009), read/write memory devices (Allwood, Xiong, and Cowburn, 2006a, 2006b), signal fan-out devices, and data input and crossover structures (Allwood et al., 2005). On the fundamental physics side, this group also investigated vortex domain wall transitions, which have a close relationship with the ferroelectric vortex domains discussed in Sec. III. They also examined magnetic comb structures (shown in Fig. 35) in detail (Lewis et al., 2010).

It is not an exaggeration to say that microelectronic devices based upon magnetic domain dynamics are a full decade ahead of those based upon ferroelectric domains.

In some important respects, it is difficult for ferroelectrics to catch up, literally. This is because magnetic domain walls involve only flipping of spins (no mass) and can easily be driven at near the speed of sound $(\mathrm{km} / \mathrm{s})$. In fact, they can even be driven supersonically, with acoustic phonons being produced in high magnetic fields by supersonic magnetic domain walls at a phase angle related to that in the analogous problems of bow waves in water or in Cerenkov radiation (Demokritov et al., 1988, 1991). By comparison, ferroelectric walls have real momentum and they cannot travel faster than the theoretical limit set by the transverse acoustic phonon, the speed of sound, for otherwise the sonic boom would shatter the crystal. Moreover, domain walls satisfy a ballistic 
(a)
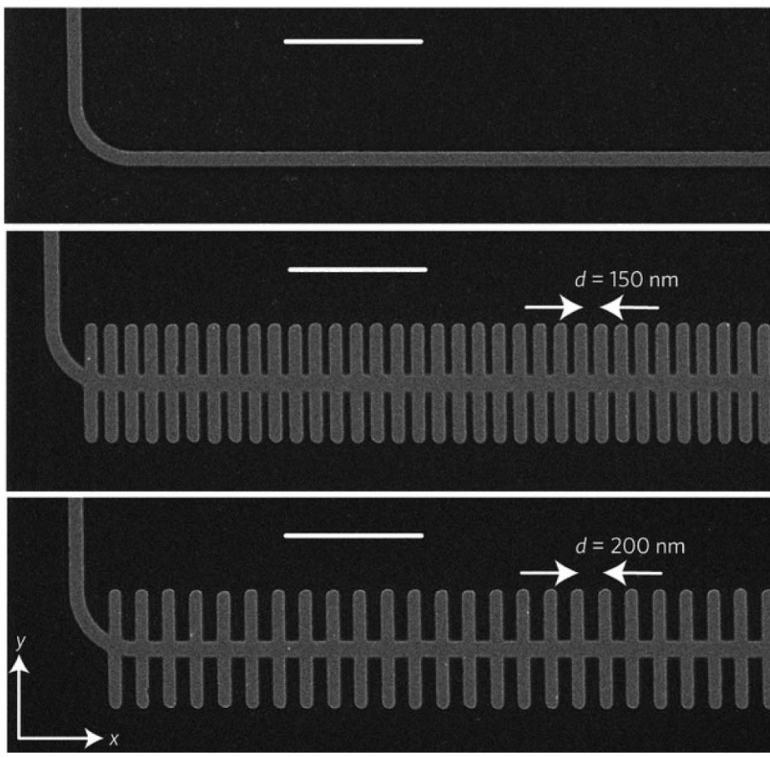

(b)



FIG. 35. (a) Electron microscope images of a magnetic strip and magnetic comb structures; the scale bar is $1 \mu \mathrm{m}$ in all cases. Magnetic comb structures are designed to speed up domain walls, as experimentally demonstrated in (b). Note the high velocity of the magnetic domain walls (in excess of $1500 \mathrm{~m} / \mathrm{s}$ ) that can be achieved in the combed structures. From Lewis et al., 2010.

equation of motion with viscous damping (Dawber et al., 2005 ) which causes a saturation or "terminal velocity" that is often (but not always) below the speed of sound.

On the other hand, the terminal velocity, however, is dependent on the sample and the technique used to deliver the voltage pulse, and it can be significantly raised. Miller measured ferroelectric domain wall velocities in $\mathrm{LiNbO}_{3}$ over 9 orders of magnitude from $10^{-9} \mathrm{~mm} / \mathrm{s}$ upward and found that they saturate near $1.0 \mathrm{~m} / \mathrm{s}$ at high fields (Miller, 1998). But more recent studies by Gruverman set a higher limit, between 10 and $100 \mathrm{~m} / \mathrm{s}$ (Gruverman, Wu, and Scott, 2008). And much faster switching, with velocities approaching the speed of sound, was achieved by $\mathrm{Li}$ and co-workers in direct measurements of the switching dynamics using an ultrafast photoconducting switch enabled electric pulse with a rise time of tens of picoseconds (Li et al., 2004). They showed intrinsic switching time scales of 50-70 psec in fully integrated capacitors of $2 \times 3 \mu \mathrm{m}^{2}$ in lateral dimensions, fabricated with films of $150 \mathrm{~nm}$ thickness, suggesting velocities of $2000-3000 \mathrm{~m} / \mathrm{s}$. It should be noted that such measurements are not routine: Indeed, it is quite likely that a significant number of measurements in the literature are compromised by either the rise time of the pulse generator, the $R C$ time constant of the measurement system, or the rise time of the oscilloscope system. It is also worth mentioning that ultrahigh fields can be achieved in sufficiently thin single-crystal samples: Morrison et al. (2005) reported fields of $1.3 \mathrm{GV} / \mathrm{m}$ in $\mathrm{BaTiO}_{3}$ lamellae, and nobody knows what the domain wall speed is under such high fields.

As well as maximizing domain wall speed, the future development of ferroelectric domain wall devices will probably require denser domain arrays, so that the walls travel a shorter distance, or rely on device designs that do not require high wall speeds, such as domain conduction devices. The first criterion can be readily met, since ferroelectric domains are known to be generally narrower than their magnetic counterparts, as seen in previous sections. The second (conductivity of domain walls) has also been discussed and will be examined further in the next section.

Another important question, which is only now beginning to be explored, is that of domain wall dynamics in magnetoelectric multiferroics. The different response of the magnetic and ferroelectric components of multiferroic walls to external fields has been proposed by Fontcuberta and co-workers as a new mechanism for eliciting switchable control of exchange bias in hexagonal multiferroics such as $\mathrm{YMnO}_{3}$ (Skumryev et al., 2011). On the theoretical front, little is yet known about the dynamics of coupled domain walls, so this line of work certainly merits further attention.

Parkin and co-workers have made considerable progress in memory devices based upon magnetic domain wall motion, introducing the concept of the racetrack memory (Fig. 36). This design concept in principle offers storage densities that are larger than conventional solid-state memory devices such as flash memory with a better read and write performance. Key in these devices is the fact that magnetic domain walls can exhibit considerable momentum, moving about a micron after a current pulse is applied (Thomas, Moriya, and Rettner, 2010). This is about an order of magnitude less than the inertial travel distance of ferroelectric domain walls subjected to large pulsed fields, but it is not negligible. This is important for magnetic domain memories because it implies that the spatial positioning of walls can be precisely controlled by the current pulse length. (This is somewhat surprising, since the magnetic domain motion follows the Landau-Lifshitz-Gilbert equation, which is first order in time, whereas ferroelectric domain walls satisfy Newton's equations, which are second order in time and hence explicitly display momentum.)

The basic mechanism to "push" domain walls along the racetrack using a current is the "spin torque." The underlying principle is that when spin-polarized electrons in a ferromagnetic material pass through a magnetic domain wall, there is a torque on the electrons that acts to reorient their spin magnetic moments along the magnetization direction (Fig. 37). Angular momentum in this system is conserved by a reaction torque, termed the spin transfer torque, which acts from the 


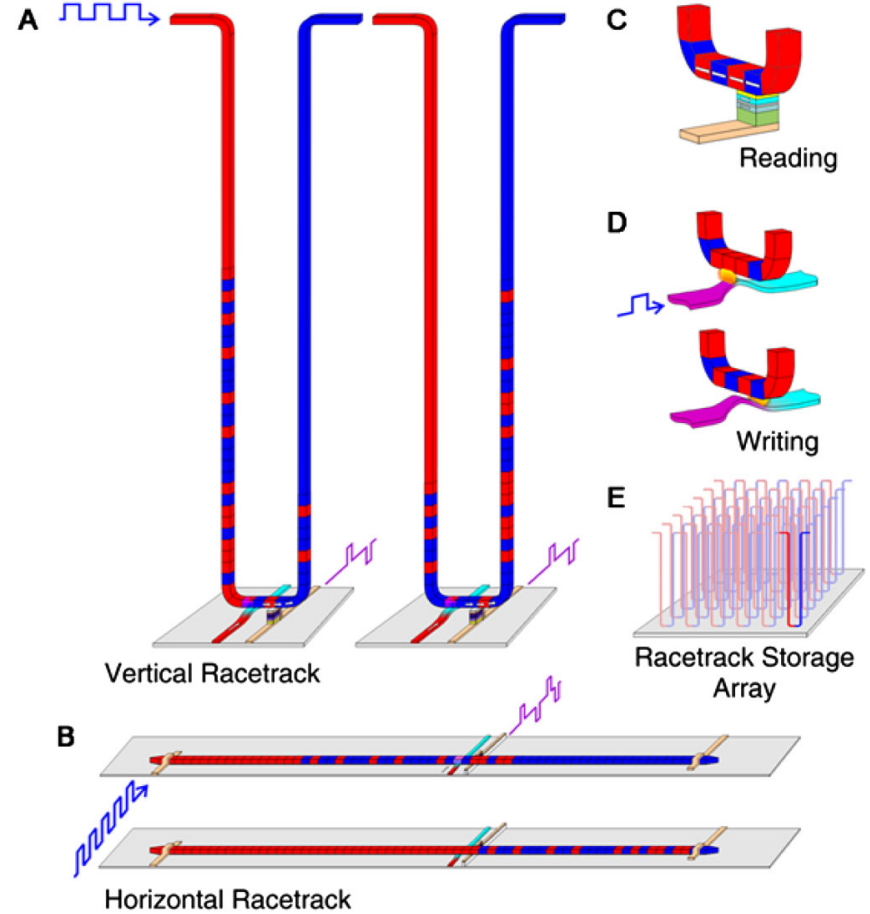

FIG. 36 (color online). The racetrack: a ferromagnetic nanowire. Pulses of highly spin-polarized current move domain walls coherently in either direction via spin torque. (a) A vertical-configuration racetrack. Magnetic patterns in the racetrack before and after the domain walls have moved down one branch of the $U$, past the read and write elements, and then up the other branch. (b) A horizontal configuration. (c) Reading data from the stored pattern by measuring the tunnel magnetoresistance of a magnetic tunnel junction element connected to the racetrack. (d) Writing data by the fringing fields of a domain wall moved in a second ferromagnetic nanowire. (e) Arrays of racetracks on a chip for high-density storage. From Parkin, Hayashi, and Thomas, 2008.

electrons onto the material magnetization in a way such that it displaces the domain wall in the direction of the electron flow (Stiles and Miltat, 2006; Ralph and Stiles, 2008). Domain wall motion can be achieved when the current density through the device is sufficiently high.

Current designs of racetrack memories use a spin-coherent electric current to move magnetic domains along a nanoscopic permalloy wire with a cross section of $200 \times 100 \mathrm{~nm}^{2}$. As a current is passed through the wire, the domain walls pass by read and write heads. A memory device is made from

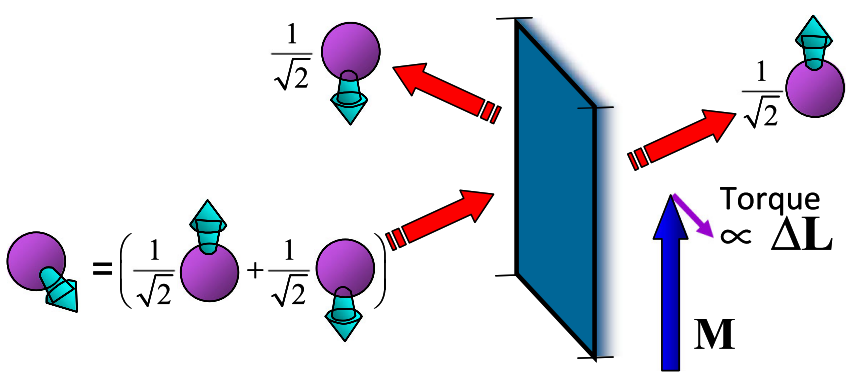

FIG. 37 (color online). Schematic of spin scattering from an interface with a ferromagnet in a simple limit of ideal spindependent transmission and reflection. From http://www.nist.gov/ cnst/epg/spin_transfer_torque.cfm. many such elements. Improvements in domain wall detection capabilities, based on the development of new magnetoresistive materials, allow the use of increasingly smaller magnetic domains to reach higher storage densities. The basic operation of the racetrack magnetic domain wall memory system is described by Parkin, Hayashi, and Thomas (2008), and recent details concerning wall pinning discussed by Jiang et al. (2010), although the basic idea that magnetic domain walls could be moved into precise positions was developed a decade earlier by Ono et al. (1999a, 1999b). A recent review of this aspect of magnetic electronics ("spintronics") has been given by Bader and Parkin (2010), which follows an earlier review on fundamentals and applications of nanomagnetism by Bader (2006).

\section{F. Emergent aspects of domain wall research}

We focus on some emergent behavior at domain walls, particularly in materials such as multiferroics, that exhibit coupled order parameters, i.e., the charge and spin degree of freedom are coupled. In order to focus the discussion on what is understood and what remains to be explored, we use the multiferroic $\mathrm{BiFeO}_{3}$ as our model system. The richness of phase evolution and electronic properties in this system is now well established, and we are beginning to understand the manipulation of its electronic structure, correlation effects, and order parameter evolution on the unit-cell level. What are the consequences and the opportunities? This we discuss next.

\section{Conduction properties, charge, and electronic structure}

By far one of the most fascinating aspects of research on a bismuth ferrite as a multiferroic has to do with the changes in electronic structure as a function of crystal chemistry and particularly at domain walls. Rhombohedral $\mathrm{BiFeO}_{3}$ has been shown to possess ferroelectric domains in thin films that are insulatorlike, whereas conduction in its domain walls is significant (Seidel et al., 2009) (Fig. 38). The observed conductivity correlates with structurally driven changes in both the electrostatic potential and the local electronic structure, which shows a decrease in the band gap at the domain wall.

In light of the intriguing electrical conductivity, detailed electronic properties of the domain walls have been investigated by Lubk, Gemming, and Spaldin (2009). The layerby-layer densities of states was calculated to see if the structural deformations in the wall region lead to a closing of the electronic band gap. In particular, the ideal cubic structure, in which the $180^{\circ} \mathrm{Fe}-\mathrm{O}-\mathrm{Fe}$ bond angles maximize the Fe $3 d-\mathrm{O} 2 p$ hybridization and hence the bandwidth, has a significantly reduced band gap compared to the $R 3 c$ structure. Figure 39 shows the local band gap extracted from the layerby-layer densities of states across the three wall types. In all cases a reduction in the band gap in the wall can be seen, with the $180^{\circ}$ wall showing the largest effect. In no case, however, does the band gap approach zero in the wall region. The same first-principles calculations supporting the experimental work of Seidel et al. also give insight into the changes in the $\mathrm{Fe}-\mathrm{O}-\mathrm{Fe}$ bond angle in $\mathrm{BiFeO}_{3}$, in addition to the fact that walls in which the rotations of the oxygen octahedra do not 


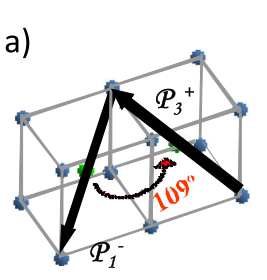

b)

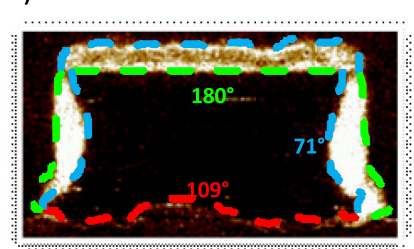

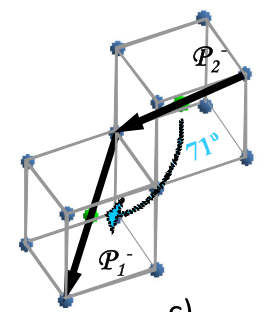

c)

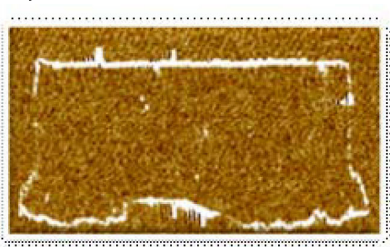

FIG. 38 (color online). (a) The three different types of domain walls in rhombohedral bismuth ferrite. Arrows indicate polarization directions in adjacent domains. (b) In-plane PFM image of a written domain pattern in a monodomain BFO (110) film showing all three types of domain wall. (c) Corresponding c-AFM image showing conduction at both $109^{\circ}$ and $180^{\circ}$ domain walls; note the absence of conduction at the $71^{\circ}$ domain walls. This stands in contrast with recent results reporting enhanced conductivity in the $71^{\circ}$ walls (Farokhipoor and Noheda, 2011). Adapted from Seidel et al., 2009.

change their phase when the polarization reorients are significantly more favorable than those with rotation discontinuities, i.e., antiphase octahedral rotations are energetically costly.

The analysis of the local polarization and electronic properties also revealed steps in the electrostatic potential for all wall types, and these must also contribute to the conductivity. Steps in the electrostatic potential at domain walls are correlated with (and caused by) small changes in the component of the polarization normal to the wall (Seidel et al., 2010). These changes in normal polarization are a consequence of the fair rotation of the polar vector across the domain wall and are not exclusive of $\mathrm{BiFeO}_{3}$. Tetragonal $\mathrm{PbTiO}_{3}$, for example, shows a similar effect for a $90^{\circ}$ wall (Meyer and Vanderbilt, 2002) (Fig. 40). Extended phase-field calculations for tetragonal $\mathrm{BaTiO}_{3}$ also allow calculating the intrinsic electrostatic potential drop across the $90^{\circ}$ domain wall, regardless of the consideration of the ferroelectric as an $n$-type semiconductor

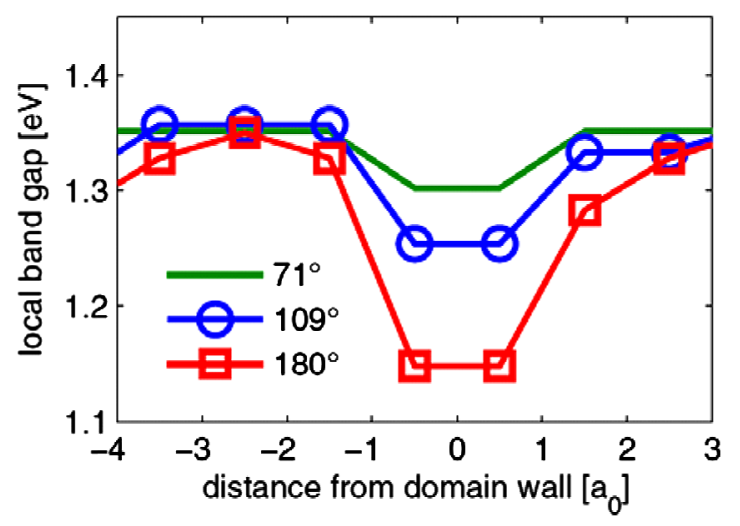

FIG. 39 (color online). Local band gap at domain walls in bismuth ferrite extracted from the layer-by-layer densities of states. From Lubk, Gemming, and Spaldin, 2009. or dielectric (Hong et al., 2008). This potential change creates a large electric field that promotes an asymmetric charge distribution around the walls, where electrons and oxygen vacancies concentrate on the opposite sides. The increased charge density presumably promotes increased conductivity.

As mentioned, the semirigid rotation of the polar vector across a ferroelectric-ferroelastic wall leads to an electrostatic potential that is screened by free charges which enhance the local charge density and thus, presumably, the conductivity. Since this polar rotation is not exclusive of $\mathrm{BiFeO}_{3}$, other perovskite ferroelectrics should also be expected to display enhanced conductivity, and perhaps this mechanism is behind the enhanced conductivity recently reported also for the domain walls of $\mathrm{Pb}(\mathrm{Zr}, \mathrm{Ti}) \mathrm{O}_{3}$ (Guyonnet et al., 2011). In $\mathrm{BiFeO}_{3}$, several other factors might be further helping the conductivity enhancement: First, the magnetoelectric coupling between polarization and spin lattice is such that the magnetic sublattice rotates with the polarization (Zhao et al., 2006; Lebeugle et al., 2008). Since spins rotate rigidly (see Sec. III.B), they might favor a more rigid rotation of the polarization and hence a bigger electrostatic step at the wall (and, of course, the polarization of $\mathrm{BiFeO}_{3}$ is itself bigger than that of other known perovskite ferroelectrics, which means that all other things being equal a rigid polar rotation in $\mathrm{BiFeO}_{3}$ will cause a bigger electrostatic step). Also, the increased spin alignment at the wall should lower the magnetic contribution to the band gap (Dieguez and Iñiguez, 2011). But perhaps the most obvious consideration is the fact that $\mathrm{BiFeO}_{3}$ has an intrinsically smaller band gap than other prototypical perovskite ferroelectrics $(\sim 2.7 \mathrm{eV}$ instead of 3.5-4 eV). This means that the screening charges accumulated at the wall will be closer to the bottom of the conduction band and hence will more easily contribute to the conductivity. It would be interesting to see if highly insulating

(a)

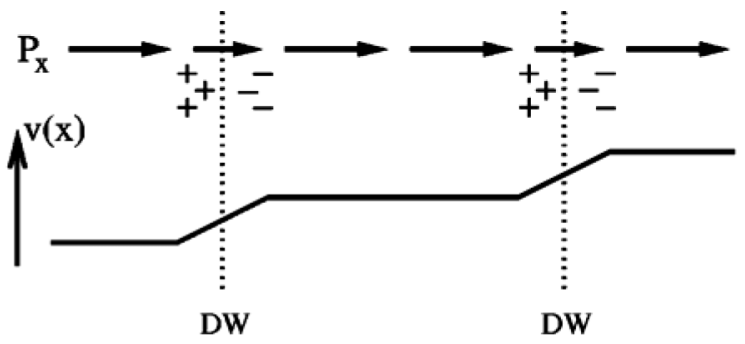

(b)



(c)

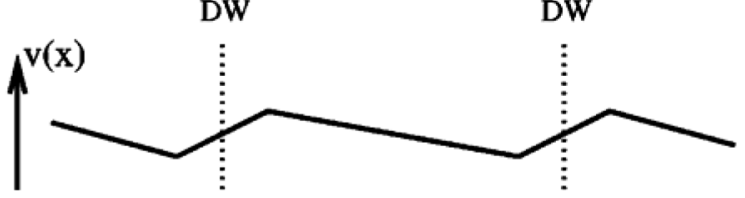

FIG. 40. $90^{\circ}$ domain walls in lead titanate: (a) potential steps at domain walls; (b) theoretical conduction and valence band alignment; (c) potential in equilibrium. From Meyer and Vanderbilt, 2002. 
single-crystal samples such as those studied by Chishima et al. (2010) display the same domain wall conductivity as do the thin-film samples studied so far. The current density of these single-crystal samples can be as low as $10^{-9} \mathrm{~A} / \mathrm{cm}^{2}$ even at electric fields in excess of $50 \mathrm{kV} / \mathrm{cm}$, while typical resistivities of thin films are in the region of $10^{6}-10^{8} \Omega \mathrm{cm}$, comparable to the resistivity of good quality $\mathrm{BiMnO}_{3}$ (Eerenstein et al., 2005).

The role of defect accumulation at the walls also deserves close scrutiny, because defects control the transport behavior, as recently emphasized by Farokhipoor and Noheda (2011). Localized states are found in the spectrum of ferroelectric semiconductors, and states localized at the walls and inside the domain but close to the wall split off from the bulk continuum. These nondegenerate states have a high dispersion, in contrast with the "heavy-fermion" states at an isolated domain wall (Idlis and Usmanov, 1992). Charged double layers can be formed due to coupling between polarization and space charges at ferroelectric-ferroelastic domain walls (Xiao et al., 2005). Charged domain wall energies are about 1 order larger than the uncharged domain wall energies (Gureev, Tagantsev, and Setter, 2009), and phenomenological calculations show decoration of walls by defects such as oxygen vacancies. The presence of charge and defect layers at the walls means that such walls promote electrical failure by providing a high conductivity pathway from electrode to electrode (Xiao et al., 2005). Eliseev et al. (2011) have also shown how the relative sign between the wall charge and the type of majority carriers also matters: positively charged walls in an $n$-type ferroelectric are more easily screened (and thus have smaller thickness and lower energy) than negatively charged walls, due to the bigger abundance of screening charges. Vice versa for negatively charged walls in a $p$-type ferroelectric semiconductor.

The control of the electronic structure at walls by doping and strain in ferroelectric and ferroeleastic oxides opens a way to effectively engineer nanoscale functionality in such materials. For the case of $\mathrm{BiFeO}_{3} A$-site doping with $\mathrm{Ca}$, and magnetic $B$-site substitution such as $\mathrm{Co}$ or $\mathrm{Ni}$, might prove to be a viable way to achieve new domain wall properties by manipulating the electronic structure, spin structure, and dipolar moment in this material (Yang et al., 2009). Of obvious future interest is the question of what sets the limits to the current transport behavior at walls: Can one "design" the topological structure of the domain wall to controllably induce electronic phase transitions within the wall arising from the correlated electron nature? Is it possible to trigger an Anderson transition by doping of domain walls or straining them?

Recently, some of us reported the observation of tunable electronic conductivity at domain walls in La-doped BFO linked to oxygen vacancy concentration (Seidel et al., 2010). The conductivity at $109^{\circ}$ walls is thermally activated with activation energies of 0.24 to $0.5 \mathrm{eV}$. From a broader perspective, these results are the first step toward realizing the tantalizing possibility of inducing an insulator-metal transition (Imada, Fujimori, and Tokura, 1998) locally within the confines of the domain wall through careful design of the electronic structure, the state of strain, and chemical effects at the domain wall. For actual device applications the magnitude of the wall current needs to be increased. The choice of the right shallow-level dopant and host material might prove to be key factors in this respect. Further study of correlations between local polarization and conductivity is an exciting approach to understanding the conduction dynamics and associated ferroelectric properties in the presence of strong coupling between electronic conduction and polarization in complex oxides.

\section{Domain wall interaction with defects}

Defect-domain wall interaction is an important area of research that deserves increased attention (Robels and Arlt, 1993; Gopalan, Dierolf, and Scrymgeour, 2007). Point defects can broaden the wall (Shilo, Ravichandran, and Bhattacharya, 2004; Lee, Salje, and Bismayer, 2005). The width of twin walls in $\mathrm{PbTiO}_{3}$, for example, can be strongly modified by the presence of point defects within the wall. The intrinsic wall width of $\mathrm{PbTiO}_{3}$ is about $0.5 \mathrm{~nm}$, but clusters of point defects can increase the size of the twin wall up to $15 \mathrm{~nm}$ (Salje and Zhang, 2009). Trapped defects at the domain boundary play a significant role in the spatial variation of the antiparallel polarization width in the $\mathrm{BaMgF}_{4}$ single crystal as seen by PFM (Zeng et al., 2008), and asymmetric charge distribution around $90^{\circ}$ domain walls in $\mathrm{BaTiO}_{3}$ have also been reported, where electrons and oxygen vacancies concentrate on the opposite sides (Hong et al., 2008).

Interaction between the order parameter and the point defect concentration causes point defects to accumulate within twin walls (Salje and Zhang, 2009); conversely such defects contribute to the twin-wall kinetics and hysteresis, as they tend to clamp the walls. Oxygen vacancies, in particular, have been shown to have a smaller formation energy in the domain wall than in the bulk, thereby confirming the tendency of these defects to migrate to, and pin, the domain walls (He and Vanderbilt, 2003). This leads to a mechanism for the domain wall to have a memory of its location during annealing (Xiao et al., 2005).

\section{Magnetism and magnetoelectric properties of multiferroic domain walls}

An important question to ask at this point is what is the true state of magnetism at a multiferroic domain wall. Temperature-dependent transport measurements are a possible route to follow to understand the actual spin structure and whether it exhibits a glasslike or ordered ferromagnetic state. Of interest is the effect of extra carriers introduced into the system, e.g., by doping or electric gating, on magnetism. Is there a way to change the magnetic interaction from superexchange to double exchange? The strength of the coupling between the ferroelectric and antiferromagnetic walls in $\mathrm{BiFeO}_{3}$ is an issue that still needs to be resolved from both a theoretical and an experimental perspective. The role of the dimensionality on electrical and magnetoelectrical transport needs to be elucidated and compared to known systems, such as manganites (Dagotto, 2003; Salafranca, Yu, and Dagotto, 2010). We note that the interaction between ferroelectric and antiferromagnetic domain walls has been studied in model multiferroics such as $\mathrm{YMnO}_{3}$ (Goltsev et al., 2003) and $\mathrm{BiFeO}_{3}$ (Gareeva and Zvezdin, 2011). In both cases it has 
been shown that the antiferromagnetic domain walls are significantly wider (by $\sim 1-2$ orders of magnitude) compared to the ferroelectric walls. This is also in agreement with the phenomenological predictions of Daraktchiev, Catalan, and Scott (2010) for coupling-mediated wall broadening.

\section{FUTURE DIRECTIONS}

It is safe to say that the phenomena and physics of domain walls in ferroelectrics form an exciting and growing field of interest. As device size is reduced, the number density of domain walls grows, with consequences for functional behavior. Based on the differences between domain wall types, the inclusion of a "wrong" domain type could give the system entirely different properties. With the current developments surrounding the conductive properties there are many remaining questions and some new ones. Electronic conduction was predicted for ferroelectric domain walls based on the fact that charged double layers may form on either side of these walls (Hong et al., 2008). Since the ferroelectric in which the conductive walls were found is a multiferroic, the obvious next step is to verify that multiferroics also have this double layer and determine whether it is responsible for the conduction. If this double layer is indeed present and responsible for the conduction, it should be interesting to combine this with the idea that multiferroics have broader walls compared to pure ferroelectrics. Is there a maximum on the thickness of a domain wall to still have this double layer and be conductive? In a more general sense one could ask oneself whether the physics and assumptions based on the findings in pure ferroelectrics are valid for the multiferroic materials as well. Conversely, we need to also identify the aspects of domain wall behavior that are exclusive to multiferroics: Order parameter coupling and chirality are two features of multiferroic walls that have unique roles.

Another front of research is the investigation of dynamic conductivity at domain walls (Maksymovych et al., 2011). This addresses important factors: a possible electric-field induced distortion of the polarization structure at the domain wall, the dependence of conductivity on the degree of distortion, and weak-pinning scenarios of the distorted wall. The domain wall is likely not a rigid electronic conductor, instead offering a quasicontinuous spectrum of voltage-tunable electronic states (Maksymovych et al., 2011). This is different from ferroelectric domains, where switching may give rise to discrete (often only two) conductance levels (Garcia et al., 2009; Maksymovych et al., 2009). The intrinsic dynamics of domain walls and other topological defects are expected not only to influence future theoretical and experimental interpretations of the electronic phenomena, but also to pose the possibility of finding unique properties of multiferroic domain walls, e.g., magnetization and magnetoresistance within an insulating antiferromagnetic matrix (He et al., 2011), also due to order parameter coupling and localized secondary order parameters (Salje and Zhang, 2009; Daraktchiev, Catalan, and Scott, 2010). Of future interest is the question of what sets the limits to the current transport behavior at walls: Can one design the topological structure of the domain wall to controllably induce electronic phase transitions within the wall arising from the correlated electron nature? Is it possible to trigger an Anderson transition by doping of domain walls (Yang et al., 2009) or straining them? The observation of superconductivity in ferroelastic walls of $\mathrm{WO}_{3}$ certainly points to various exciting and unexplored areas of domain boundary physics (Aird and Salje, 1998).

Another interesting direction for domain wall engineering in ferroelectrics is by the size and design of the system, and this includes not only the domains themselves but also their hierarchical self-organization into bigger metastructures. Recently, Schilling et al. (2009) presented work on nanoferroelectrics, which shows considerably more domain walls per unit volume, thanks to the size constraints in two and three dimensions, as opposed to the single finite dimension of thin films. Yet another unique design feature of the samples of Schilling et al. is that they are free-standing ferroelectrics, unlike those that are grown on a substrate, and for which intrinsic surface tension can play a bigger role (Luk'yanchuk et al., 2009). Such nanostructures are also prone to new types of topological defects beyond the classic domain walls; for example, recent work by Hong et al. (2010) shows that arrays of ferroelectric nanowires have switchable quadrupoles and thus potential as nanodevices. Exotic topological defects in nanostructures (vertices, vortices, quadrupoles, etc.) are currently an active area of research.

Another interesting feature that is being studied intensively is the fact that the domains in nanocrystals clearly show organization on several length scales, with correlation not just between narrow stripe domains but also between packets of stripes. Ivry et al. (2011) found a variety of mesoscopicscale domain packets or bundles with considerable cross-talk across $\mathrm{Pb}(\mathrm{Zr}, \mathrm{Ti}) \mathrm{O}_{3}$ (PZT) grain boundaries. More strikingly, McQuaid et al. (2011) showed metadomains or superdomains that are composed of thin stripes but reproduce on a mesoscopic scale the exact shape and functional behavior of closure domains such as those of Figs. 10 and 11. The physics and functional engineering of mesoscopic metadomains or bundles is a rapidly growing topic that will no doubt see more activity in the near future.

Several applications have been suggested to make use of domain walls in ferroelectric materials based on their additional functionalities as well as their affects on existing devices. Uses that have been mentioned are as a local strain sensor incorporated on an AFM probe or a multilevel resistance-state device that is written by an electrical current (Béa and Paruch, 2009). Other possibilities include nonvolatile memories, piezoelectric actuators, ultrasound transducers, surface acoustic wave devices, and optical applications (Gopalan, Dierolf, and Scrymgeour, 2007). For existing devices, the discovery of conducting domain walls stimulates engineers to prevent their products from having the wrong domain walls that could cause leakage and prevent its use in ferroelectric memories. This use of ferroelectrics in memory has recently been reviewed, and it has been argued that conductivity may not be a detriment but an opportunity for new memory reading mechanisms (Béa and Paruch, 2009; Garcia et al., 2009; Maksymovych et al., 2009; Zubko and Triscone, 2009; Jiang et al., 2011).

Experimental results and theoretical investigations in recent years have convincingly demonstrated that certain transition metal oxides and some other materials have 
dominant properties driven by spatial inhomogeneity. Strongly correlated materials incorporate physical interactions (spin, charge, lattice, and/or orbital hybridization), allowing complex interactions between electric and magnetic properties, resulting in ferromagnetic, or antiferromagnetic phase transitions. Of even higher interest are the heterointerfaces formed between correlated materials showing new state properties. Domain walls are only one example of "naturally" occurring interfaces in such materials. The challenge is to determine whether such complex interactions can be controlled in those materials or heterointerfaces at sufficiently high speeds and densities to enable new logic device functionality at the nanometer scale. Parameters such as interface energy, switching speed and threshold, tunability, dynamics of the states, and size dependencies need to be quantified to determine if domain boundary materials could be employed as a building block for information processing systems.

In addition, there are some new phenomena associated with ferroelectric domain walls that merit fundamental study: As shown in Sec. III, 2D arrays of vertex domains on ferroelectric surfaces often come in pairs of threefold vertices (Srolovitz and Scott, 1986). Fourfold vertices of domains exist in barium sodium niobate, and sixfold domains are well known since 1967 in $\mathrm{YMnO}_{3}$ (Safrankova, Fousek, and Kizhaev, 1967). And so one might ask whether these arrays of vertex domains "melt" at temperatures below the Curie temperature at which stripe domains disappear, i.e., do ferroelectric vertex arrays undergo Kosterlitz-Thouless melting (Kosterlitz and Thouless, 1973) involving defect pair production and annihilation? A second and rather deep phenomenon has been recently discovered by Schilling et al. (2011): Vertex domains in rectangular ferroelectrics have offcentered vertices, whose position can be calculated according to a Landau theory with aspect ratio replacing temperature; the resulting novel shape-generated phase transition can therefore occur at $0 \mathrm{~K}$ (quantum criticality).

The ferromagnetic properties of ferroelectric walls in paramagnetic and antiferromagnetic materials (Goltsev et al. (2003); Daraktchiev, Catalan, and Scott (2008; 2010) suggest that much more research and development should be done on domain walls in multiferroics and also on the dynamics of domain walls in these materials (Skumryev et al., 2011): We note in this respect that $\mathrm{BiFeO}_{3}$ is no longer the only roomtemperature multiferroic, nor $\mathrm{Cr}_{2} \mathrm{O}_{3}$ the only good roomtemperature magnetoelectric, with the lead-iron-tantalate, lead-iron-niobate, lead-iron-tungstate family now being studied in various laboratories (Josef Stefan Institute, University of Puerto Rico, University of Cambridge), and new chromates being reported at Florida State, all of which function at room temperature. Many of these are relaxorlike systems and therefore have intrinsic nanodomains.

In summary, we have provided an overview on ferroelectric and multiferroic nanodomain and domain wall electronics. The state of understanding and especially of application lags that for magnetic domains, with which comparisons are made; the work of Cowburn et al. and Parkin et al. makes it hard for ferroelectric domain electronics to compete with magnetic devices based on spatial manipulation; this is simply because the magnetic domains have greater mobilities. Thus it is unlikely that ferroelectrics will provide the equivalent of racetrack memories, or fast AND or NOT gates, as developed by those groups. Instead it is likely that they will provide complementary devices that exploit the electrical conductivity and/or ferromagnetisn of ferroelectric domain walls. Hence these may involve fewer memory devices but more interconnects, switches, and sensors and actuators. Domain wall electronics, particularly with ferroelectrics and multiferroics, may also provide useful hybrid devices involving carbon nanotubes (Kumar, Scott, and Katiyar, 2011).

It is always risky to predict the next generation of devices, but it is likely that ferroelectric nanodomains and domain walls may first find commercial application not in consumer electronics but in high-end products. Medical physics (particularly implants), satellite physics (NASA reported in August 2011 its test results of PZT 94. Ferroelectric random access memories (FRAMs) in microsatellites), and military applications all pay a premium for smaller size and lower power. Nanoscience has yet to live up to the publicity and hype it has received, but such initial applications, where cost is less important than size and power consumption, will surely lead the way as disruptive technologies.

\section{REFERENCES}

Aguado-Fuente P., and J. Junquera, 2008, Phys. Rev. Lett. 100, 177601

Ahn, S. J., J.-J. Kim, J.-H. Kim, and W.-K. Choo, 2003, J. Korean Phys. Soc. 42, S1009.

Aird, A., and E. K. H. Salje, 1998, J. Phys. Condens. Matter 10, L377.

Aizu, K., 1970, Phys. Rev. B 2, 754.

Allen, L. J., W. McBride, N.L. O'Leary, and M.P. Oxley, 2004, Ultramicroscopy 100, 91.

Allwood, D. A., G. Xiong, and R. P. Cowburn, 2006a, Appl. Phys. Lett. 89, 102504.

Allwood, D. A., G. Xiong, and R. P. Cowburn, 2006b, J. Appl. Phys. 100, 123908.

Allwood, D. A., G. Xiong, C. C. Faulkner, D. Atkinson, D. Petit, and R. P. Cowburn, 2005, Science 309, 1688.

Anbusathaiah, V., D. Kan, F. C. Kartawidjaja, R. Mahjoub, M. A. Arredondo, S. Wicks, I. Takeuchi, J. Wang, and V. Nagarajan, 2009, Adv. Mater. 21, 3497.

Andrews, S., and R. Cowley, 1986, J. Phys. C 19, 615.

Anikiev, A., L. G. Reznik, B.S. Umarov, and J.F. Scott, 1985, Ferroelectr., Lett. Sect. 3, 89.

Araujo, C., J. F. Scott, R. B. Godfrey, and L. McMillan, 1986, Appl. Phys. Lett. 48, 1439.

Arlt, G., 1990, J. Mater. Sci. 25, 2655.

Arlt, G., and P. Sasko, 1980, J. Appl. Phys. 51, 4956.

Armstrong, J. A., N. Bloembergen, J. Ducuing, and P. S. Pershan, 1962, Phys. Rev. 127, 1918.

Arnold, D. C., K. S. Knight, G. Catalan, S. A. T. Redfern, J. F. Scott, P. Lightfoot, and F. D. Morrison, 2010, Adv. Funct. Mater. 20, 2116.

Atkinson, D., C.C. Faulkner, D. A. Allwood, and R.P. Cowburn, 2006, Topics Appl. Phys. 101, 207.

Avrami, M., 1939, J. Chem. Phys. 7, 1103.

Bader, S. D., 2006, Rev. Mod. Phys. 78, 1.

Bader, S. D., and S. S. P. Parkin, 2010, Annu. Rev. Condens. Matter Phys. 1, 71.

Balke, N., S. Choudhury, S. Jesse, M. Huijben, Y.H. Chu, A.P. Baddorf, L. Q. Chen, R. Ramesh, and S. V. Kalinin, 2009, Nature Nanotech. 4, 868. 
Balke, N., et al., 2011, Nature Phys. 8, 81.

Bartels, M., V. Hagen, M. Burianek, M. Getzlaff, U. Bismayer, and R. Wiesendanger, 2003, J. Phys. Condens. Matter 15, 957.

Bassiri-Gharb, N., I. Fujii, E. Hong, S. Trolier-McKinstry, D. V. Taylor, and D. Damjanovic, 2007, J. Electroceram. 19, 49.

Batchko, R. G., V. Y. Shur, M. M. Fejer, and R. L. Byer, 1999, Appl. Phys. Lett. 75, 1673.

Batra, I., and B. Silverman, 1972, Solid State Commun. 11, 291.

Béa, H., and P. Paruch, 2009, Nature Mater. 8, 168.

Bierlein, J.D., and H. van Herzeele, 1989, J. Opt. Soc. Am. B 6, 622.

Bjorkstam, J.L., and R.E. Oettel, 1967, Phys. Rev. 159, 427.

Blank, H., and S. Amelinckx, 1963, Appl. Phys. Lett. 2, 140.

Blinc, R., B. Zalar, V. V. Laguta, and M. Itoh, 2005, Phys. Rev. Lett. 94, 147601.

Bokov, A. A., and Z. -G. Ye, 2006, J. Mater. Sci. 41, 31.

Borisevich, A. Y., et al., 2010, ACS Nano 4, 6071.

Bornarel, J., J. Lajzerowicz, and J. F. Legrand, 1974, Ferroelectrics 7, 313.

Bratkovsky, A. M., and A.P. Levanyuk, 2000, Phys. Rev. Lett. 84, 3177.

Brown, M.E., and M.D. Hollingsworth, 1995, Nature (London) 376, 323.

Bruce, D. A., 1981, J. Phys. C 14, 5195.

Burns, G., and F. H. Dacol, 1982, Solid State Commun. 42, 9.

Bursill, L. A., and Peng Ju Lin, 1986, Ferroelectrics 70, 191.

Bursill, L. A., J.L. Peng, and D. Feng, 1983, Philos. Mag. A 48, 953.

Canalias, C., V. Pasiskevicius, and F. Laurell, 2005, Appl. Phys. Lett. 86, 181105

Canalias, C., V. Pasiskevicius, and F. Laurell, 2006, Ferroelectrics 340, 27.

Canalias, C., S. Wang, V. Pasiskevicius, and F. Laurell, 2006, Appl. Phys. Lett. 88, 032905

Cano, A., and A. P. Levanyuk, 2010, Phys. Rev. B 81, 172105.

Cao, W., and G. R. Barsch, 1990, Phys. Rev. B 41, 4334.

Cao, W., and C. A. Randall, 1996, J. Phys. Chem. Solids 57, 1499.

Catalan, G., H. Béa, S. Fusil, M. Bibes, P. Paruch, A. Barthélémy, and J. F. Scott, 2008, Phys. Rev. Lett. 100, 027602.

Catalan, G., I. Lukyanchuk, A. Schilling, J. M. Gregg, and J. F. Scott, 2009, J. Mater. Sci. 44, 5307.

Catalan, G., A. Schilling, J. M. Gregg, and J.F. Scott, 2007a, J. Phys. Condens. Matter 19, 022201.

Catalan, G., A. Schilling, J.F. Scott, and J.M. Gregg, 2007b, J. Phys. Condens. Matter 19, 132201.

Catalan, G., and J. F. Scott, 2009, Adv. Mater. 21, 2463.

Catalan, G., A. Lubk, A. H. G. Vlooswijk, E. Snoeck, C. Magen, A. Janssens, G. Rispens, G. Rijnders, D. H. A. Blank and B. Noheda, 2011, Nature Mater. 10, 963.

Chaib, H., T. Otto, and L. M. Eng, 2003a, Phys. Rev. B 67, 174109.

Chang, L.W., M. Alexe, J.F. Scott, and J.M. Gregg, 2009, Adv. Mater. 21, 4911.

Chen, C. H., J. M. Gibson, and R. M. Fleming, 1982, Phys. Rev. B 26, 184.

Chen, F. S., 1968, Appl. Phys. Lett. 13, 223.

Chen, F. S., 1969, J. Appl. Phys. 40, 3389.

Chen, Q., and W.P. Risk, 1994, Electron. Lett. 30, 1516.

Chen, Y. B., C. Zhang, Y. Y. Zhu, S. N. Zhu, H. T. Wang, and N. B.

Ming, 2001, Appl. Phys. Lett. 78, 577.

Cheong, S.-W., and M. Mostovoy, 2007, Nature Mater. 6, 13.

Chishima, Y., Y. Noguchi, Y. Kitanaka, and M. Miyayama, 2010,

IEEE Trans. Ultrason. Ferroelectr. Freq. Control 57, 2233.

Chiu, Ya-Ping, et al., 2011, Adv. Mater. 23, 1530.
Choi, T., Y. Horibe, H. T. Yi, Y. J. Choi, W. Wu, and S.-W. Cheong, 2010, Nature Mater. 9, 423.

Choudhury, S., J. X. Zhang, X. L. Li, L. Q. Chen, Q. X. Jia, and S. V. Kalinin, 2008, Appl. Phys. Lett. 93, 162901.

Choudhury, S., et al., 2008, J. Appl. Phys. 104, 084107.

Chowdhury, M. R., 1978, J. Phys. C 11, 1671.

Chu, S., et al., 2008, Adv. Optoelectronics 2008, 151487.

Craik, D. J., and P. V. Cooper, 1970, Phys. Lett. A 33, 411.

Cross, L. E., 1987, Ferroelectrics 76, 241.

Dagotto, Elbio, 2003, Nanoscale Phase Separation and Colossal Magnetoresistance (Springer, Berlin).

Dalton, N.W., J.T. Jacobs, and B.D. Silverman, 1971, Ferroelectrics 2, 21.

Danneau, R., et al., 2002, Phys. Rev. Lett. 88, 157201.

Daraktchiev, M., G. Catalan, and J.F. Scott, 2008, Ferroelectrics 375, 122.

Daraktchiev, M., G. Catalan, and J. F. Scott, 2010, Phys. Rev. B 81, 224118.

Daumont, C. J. M., D. Mannix, S. Venkatesan, G. Catalan, D. Rubi, B. J. Kooi, J. Th. M. De Hosson, and B. Noheda, 2009, J. Phys. Condens. Matter 21, 182001.

Daumont, C. J. M., S. Venkatesan, B. J. Kooi, J. Th. M. De Hosson, and B. Noheda, 2010, arXiv:1008.0315v3.

Davis, M., D. Damjanovic, and N. Setter, 2006, J. Appl. Phys. 100, 084103.

Dawber, M., P. Chandra, P. B. Littlewood, and J. F. Scott, 2003, J. Phys. Condens. Matter 15, L393.

Dawber, M., D. J. Jung, and J. F. Scott, 2003, Appl. Phys. Lett. 82, 436.

Dawber, M., C. Lichtensteiger, M. Cantoni, M. Veithen, P. Ghosez, K. Johnston, K. M. Rabe, and J.-M. Triscone, 2005, Phys. Rev. Lett. 95, 177601.

De Guerville, F., I. Luk'yanchuk, L. Lahoche, and M. El Marssi, 2005, Mater. Sci. Eng. B 120, 16.

Demokritov, S. O., A. I. Kirilyuk, N. M. Kreines, V. I. Kudinov, V. B. Smirnov, and M. V. Chetkin, 1988, JETP Lett. 48, 294.

Demokritov, S. O., A. I. Kirilyuk, N.M. Kreines, V.I. Kudinov, V. B. Smirnov, and M. V. Chetkin, 1991, J. Magn. Magn. Mater. 102, 339.

Dieguez, O., and J. Iñiguez, 2011 (private communication).

Dolino, G., 1973, Appl. Phys. Lett. 22, 123.

Dupé, B., I. C. Infante, G. Geneste, P.-E. Janolin, M. Bibes, A. Barthélémy, S. Lisenkov, L. Bellaiche, S. Ravy, and B. Dkhil, 2010, Phys. Rev. B 81, 144128.

Ederer, C., and J. J. Fennie, 2008, J. Phys. Condens. Matter 20, 434219.

Edlund, E., and M. N. Jacobi, 2010, Phys. Rev. Lett. 105, 137203.

Eerenstein, W., F. D. Morrison, J. F. Scott, and N. D. Mathur, 2005, Appl. Phys. Lett. 87, 101906.

Eliseev, E., et al., 2011, Phys. Rev. B 83, 235313.

Eng, L. M., 1999a, Nanotechnology 10, 405.

Farokhipoor, S., and B. Noheda, 2011, Phys. Rev. Lett. 107, 127601.

Fennie, C. J., and K. M. Rabe, 2005, Phys. Rev. B 72, 100103.

Fiebig, M., A. V. Goltsev, Th. Lottermoser, and R. V. Pisarev, 2004, J. Magn. Magn. Mater. 272-276, 353.

Fisher, D. S., 1986, Phys. Rev. Lett. 56, 1964.

Fleury, P. A., J. F. Scott, and J. M. Worlock, 1968, Phys. Rev. Lett. 21, 16.

Floquet, N., C. M. Valot, M. T. Mesnier, J. C. Niepce, L. Normand, A. Thorel, and R. Kilaas, 1997, J. Phys. III (France) 7, 1105.

Foeth, M., A. Sfera, P. Stadelmann, and P.-A. Buffat, 1999, J. Electron Microsc. 48, 717.

Fong, D. D., et al., 2006, Phys. Rev. Lett. 96, 127601. 
Fong, D. D., G. B. Stephenson, S. K. Streiffer, J. A. Eastman, O. Auciello, P. H. Fuoss, and C. Thompson, 2004, Science 304, 1650. Fousek, J., 1971, Czech. J. Phys. 21, 955.

Fousek, J., and V. Janoušek, 1966, Phys. Stat. Sol. (b) 13, 195.

Fousek, J., and V. Janovec, 1969, J. Appl. Phys. 40, 135.

Fouskova, A., 1965, Theory J. Phys. Soc. Jpn. Part II 20, 1625.

Fujii, I., M. Ugorek, and Y. Han, and S. Trolier-McKinstry, 2010, J. Am. Ceram. Soc. 93, 1081.

Galvanauskas, A., 1997, Opt. Lett. 22, 105.

Garcia, V., S. Fusil, K. Bouzehouane, S. Enouz-Vedrenne, N. D. Mathur, A. Barthelemy, and M. Bibes, 2009, Nature (London) 460, 81.

García, R.E., B. D. Huey, and J.E. Blendell, 2006, J. Appl. Phys. 100, 064105.

Gareeva, Z. V., and A. K. Zvezdin, 2011 (private communication).

Goltsev, A. V., R. V. Pisarev, Th. Lottermoser, and M. Fiebig, 2003, Phys. Rev. Lett. 90, 177204.

Gonçalves-Ferreira, L., S. A. T. Redfern, E. Artacho, and E. K. H. Salje, 2008, Phys. Rev. Lett. 101, 097602.

Goo, E. K. W., et al., 1981, J. Appl. Phys. 52, 2940.

Gopalan, V., V. Dierolf, and D. A. Scrymgeour, 2007, Annu. Rev. Mater. Res. 37, 449.

Grilli, S., P. Ferraro, P. De Natale, B. Tiribilli, and M. Vassalli, 2005, Appl. Phys. Lett. 87, 233106.

Grupp, D.E., and A. M. Goldman, 1997, Science 276, 392.

Gruverman, A., C. Isobe, and M. Tanaka, 2001, Mater. Res. Soc. Symp. Proc. 655, CC8.5.

Gruverman, A., O. Kolosov, J. Hatano, K. Takahashi, and H. Tokumoto, 1995a, J. Vac. Sci. Technol. B 13, 1095).

Gruverman, A., O. Auciello, and H. Tokumoto, 1996, J. Vac. Sci. Technol. B14, 602.

Gruverman, A., B.J. Rodriguez, C. Dehoff, J.D. Waldrep, A. I. Kingon, R. J. Nemanich, and J. S. Cross, 2005, Appl. Phys. Lett. 87, 082902.

Gruverman, A., D. Wu, H. J. Fan, I. Vrejoiu, M. Alexe, R. J. Harrison, and J. F. Scott, 2008, J. Phys. Condens. Matter 20, 342201.

Gruverman, A., D. Wu, and J. F. Scott, 2008, Phys. Rev. Lett. 100, 097601.

Gruverman, A., et al., 1995b, Phys. Rev. Lett. 74, 4309.

Gureev, T. M. Y., A. K. Tagantsev, and N. Setter, 2009, Structure and Energy of Charged Domain Walls in Ferroelectrics: Proceedings of the 18th IEEE ISAF (IEEE, New York).

Guyonnet, J., et al., 2011, Adv. Mater. 23, 5377.

He, Q., et al., 2011 (unpublished).

He, L., and D. Vanderbilt, 2003, Phys. Rev. B 68, 134103.

Hehn, M., S. Padovani, K. Ounadjela, and J. P. Bucher, 1996, Phys. Rev. B 54, 3428.

Henriksson, M., et al., 2006, Appl. Phys. B 86, 497.

Hirohashi, J., et al., 2007, J. Appl. Phys. 101, 033105.

Hlinka, J., 2008, Ferroelectrics 375, 132.

Hlinka, J., and P. Marton, 2008, Integr. Ferroelectr. 101, 50.

Hlinka, J., P. Ondrejkovic, and P. Marton, 2009, Nanotechnology 20, 105709 .

Hong, J., G. Catalan, D. N. Fang, E. Artacho, and J. F. Scott, 2010, Phys. Rev. B 81, 172101.

Hong, L., A. K. Soh, Q. G. Du, and J. Y. Li, 2008, Phys. Rev. B 77, 094104.

Houchmandzadeh, B., J. Lajzerowicz, and E. Salje, 1991, J. Phys. Condens. Matter 3, 5163.

Hu, Z. W., P. A. Thomas, and J. Webjörn, 1996, J. Appl. Crystallogr. 29, 279.

Hubert, A., and R. Schafer, 1998, Magnetic Domains (Springer, New York).

Hubert, C., and J. Levy, 1997, Appl. Phys. Lett. 71, 3353.
Huse, D. A., C. L. Henley, and D. S. Fisher, 1985, Phys. Rev. Lett. 55, 2924.

Hytch, M. J., 1998, Ultramicroscopy 74, 131.

Idlis, B. G., and M. S. Usmanov, 1992, JETP Lett. 56, 264.

Imada, M., A. Fujimori, and Y. Tokura, 1998, Rev. Mod. Phys. 70, 1039.

Ishibashi, Y., and Y. Takagi, 1971, J. Phys. Soc. Jpn. 31, 506.

Itoh, M., R. Wang, Y. Inaguma, T. Yamaguchi, Y-J. Shan, and T. Nakamura, 1999, Phys. Rev. Lett. 82, 3540.

Ivry, Y., D. Chu, and C. Durkan, 2009, Appl. Phys. Lett. 94, 162903.

Ivry, Y., D. Chu, J. F. Scott, and C. Durkan, 2011, Adv. Funct. Mater. 21, 1827.

Ivry, Y., D. P. Chu, and C. Durkan, 2010, Nanotechnology 21, 065702.

Ivry, Y., D. P. Chu, J. F. Scott, and C. Durkan, 2010, Phys. Rev. Lett. 104, 207602.

Jach, T., 2004, Phys. Rev. B 69, 064113.

Jang, H. W., et al., 2010, Phys. Rev. Lett. 104, 197601.

Janovec, V., 1983, Phys. Lett. A 99, 384.

Janovec, V., and V. Dvorak, 1986, Ferroelectrics 66, 169.

Janovec, V., and V. Dvorak, 1985, Charge Density Waves in Solids, edited by H. Araki et al., Lecture Notes in Physics Vol. 217 (Springer, Berlin).

Janovec, V., L. Richterová, and J. Privratska, 1999, Ferroelectrics 222, 73

Jaque, D., et al., 2006, Opt. Commun. 262, 220.

Jausovec, Ana-Vanessa, Gang Xiong, and Russell P. Cowburn, 2006, Appl. Phys. Lett. 88, 052501.

Jia, C. L., 2003, Science 299, 870.

Jia, C.-L., S.-B. Mi, K. Urban, I. Vrejoiu, M. Alexe, and D. Hesse, 2008, Nature Mater. 7, 57.

Jia, C. L., and K. Urban, 2004, Science 303, 2001.

Jia, C.-L., K. W. Urban, M. Alexe, D. Hesse, and I. Vrejoiu, 2011, Science 331, 1420.

Jiang, A. Q., C. Wang, K. J. Jin, X. B. Liu, J. F. Scott, C. S. Hwang, T. A. Tang, H. B. Lu, and G. Z. Yang, 2011, Adv. Mater. 23, 1277.

Jiang, X., L. Thomas, R. Moriya, M. Hayashi, B. Bergman, C. Rettner, and S. S. P. Parkin, 2010, Nature Commun. 1, 25.

Jin, L., Z. He, and D. Damjanovic, 2009, Appl. Phys. Lett. 95, 012905.

Jones, S. P. P., D. M. Evans, M. A. Carpenter, S. A. T. Redfern, J. F. Scott, U. Straube, and V.H. Schmidt, 2011, Phys. Rev. B 83, 094102.

Jungk, T, A. Hoffmann, and E. Soergel, 2006, Appl. Phys. Lett. 89, 163507.

Jungk, T., A. Hoffmann, and E. Soergel, 2007, J. Appl. Phys. 102, 084102.

Jungk, T., Á. Hoffmann, M. Fiebig, and E. Soergel, 2010, Appl. Phys. Lett. 97, 012904.

Junquera, J., and P. Ghosez, 2003, Nature (London) 422, 506.

Kalinin, S. V., et al., 2010, Rep. Prog. Phys. 73, 056502.

Kardar, M., and D. R. Nelson, 1985, Phys. Rev. Lett. 55, 1157.

Karlsson, H., 1997, Appl. Phys. Lett. 71, 3474.

Khachaturyan, A.G., 1983, The Theory of Structural Transformations in Solids, (Wiley, New York).

Kim, S., 2000, Appl. Phys. Lett. 77, 2051.

Kim, S., et al., 2005, Mater. Sci. Eng. B 120, 91.

Kinase, W., and H. Takahashi, 1957, J. Phys. Soc. Jpn. 12, 464.

Kittel, C., 1946, Phys. Rev. 70, 965.

Kopal, A., T. Bahnik, and J. Fousek, 1997, Ferroelectrics 202, 267.

Kopal, A., et al., 1999, Ferroelectrics 223, 127.

Kornev, I., H. Fu, and L. Bellaiche, 2004, Phys. Rev. Lett. 93, 196104. 
Kosterlitz, J. M., and D. J. Thouless, 1973, J. Phys. C 6, 1181.

Kostritskii, S. M., P. Bourson, R. Mouras, and M. D. Fontana, 2007, Opt. Mater. 29, 732.

Kostritskii, S. M., et al., 2008, J. Appl. Phys. 104, 114104.

Kudo, Akihiko, and Y. Miseki, 2009, Chem. Soc. Rev. 38, 253.

Kumar, A., J. F. Scott, and R. S. Katiyar (2011) (in press).

Kumar, A., G. L. Sharma, R. S. Katiyar, R. Pirc, R. Blinc, and J. F. Scott, 2009, J. Phys. Condens. Matter 21, 382204.

Kurz, J. R., X.P. Xie, and M. M. Fejer, 2002, Opt. Lett. 27, 1445.

Lagatsky, A. A., et al., 2007, Opt. Express 15, 1155.

Lajzerowicz, J., and J. J. Niez, 1979, J. Phys. Lett. 40, 165.

Landau, L., and E. Lifshitz, 1935, Phys. Z. Sowjenunion 8, 153.

Laurell, F., and C. Canalias, 2009 (private communication).

Lebeugle, D., D. Colson, A. Forget, M. Viret, A. M. Bataille, and A. Gukasov, 2008, Phys. Rev. Lett. 100, 227602.

Lee, D., R. K. Behera, P. Wu, H. Xu, Y.L. Li, S. B. Sinnott, S. R. Phillpot, L. Q. Chen, and V. Gopalan, 2009a, Phys. Rev. B 80, 060102.

Lee, D., A. Yoon, S. Y. Jang, J.-G. Yoon, J.-S. Chung, M. Kim, J. F. Scott, and T. W. Noh, 2011, Phys. Rev. Lett. 107, 057602.

Lee, W. T., and E. K. H. Salje, 2005, Appl. Phys. Lett. 87, 143110. Lee, W. T., E. K. H. Salje, and U. Bismayer, 2005, Phys. Rev. B 72, 104116.

Lemerle, S., J. Ferré, C. Chappert, V. Mathet, T. Giamarchi, and P. Le Doussal, 1998, Phys. Rev. Lett. 80, 849.

Levstik, A., V. Bobnar, C. Filipič, J. Holc, M. Kosec, R. Blinc, Z. Trontelj, and Z. Jagličić, 2007, Appl. Phys. Lett. 91, 012905.

Lewis, E. R., et al., 2010, Nature Mater. 9, 980.

Li, F., S. Zhang, Z. Xu, X. Wei, J. Luo, and T. R. Shrout, 2010, J. Appl. Phys. 108, 034106.

Li, J., B. Nagaraj, H. Liang, W. Cao, Chi. H. Lee, and R. Ramesh, 2004, Appl. Phys. Lett. 84, 1174.

Li, Yin-Yuan, 1956, Phys. Rev. 101, 1450.

Lichte, H., 2002, Ultramicroscopy 93, 199.

Lin, P. J., and L. A. Bursill, 1982, Philos. Mag. A 45, 911.

Lines, M. E., and A. M. Glass, 2004, Principles and Applications of Ferroelectrics and Related Materials (Oxford University Press, Oxford)

Little, E. A., 1955, Phys. Rev. 98, 978.

Litvin, D. B., V. Janovec, and S. Y. Litvin, 1994, Ferroelectrics 162, 275.

Liu, Tie-Qi, 2004, Ph.D. thesis, Georgia Tech.

Locherer, K. R., J. Chrosch, and E. K. H. Salje, 1998, Phase Transit. 67, 51.

Logginov, A. S., G. A. Meshkov, A. V. Nikolaev, E. P. Nikolaeva, A.P. Pyatakov, and A. K. Zvezdin, 2008, Appl. Phys. Lett. 93, 182510.

Lu, H., et al., 2011, arXiv:1110.1306.

Lubk, A., S. Gemming, and N. A. Spaldin, 2009, Phys. Rev. B 80, 104110

Luk'yanchuk, I. A., A. Schilling, J. M. Gregg, G. Catalan, and J. F. Scott, 2009, Phys. Rev. B 79, 144111.

Maggio-Aprile, I., C. Rennet, A. Erb, E. Walker, and O. Fischer, 1997, Nature (London), 390, 487.

Maksymovych, P., J. Seidel, Y.-H. Chu, A. Baddorf, P. Wu, L.-Q. Chen, S. V. Kalinin, and R. Ramesh, 2011, Nano Lett. 11, 1906.

Maksymovych, P., S. Jesse, P. Yu, R. Ramesh, A. P. Baddorf, and S. V. Kalinin, 2009, Science 324, 1421.

Mannhart, J., and D. G. Schlom, 2010, Science 327, 1607.

Marti, X., P. Ferrer, J. Herrero-Albillos, J. Narvaez, V. Holy, N. Barrett, and M. Alexe, and G. Catalan, 2011, Phys. Rev. Lett. 106, 236101.

Marton, P., I. Rychetsky, and J. Hlinka, 2010, Phys. Rev. B 81, 144125 .
Matsuura, M., H. Endoh, M. Matsushita, Y. Tachi, Y. Iwasaki, and K. Hirota, 2010, J. Phys. Soc. Jpn. 79, 033601.

McMillen, M., R. G. P. McQuaid, S.C. Haire, C. D. McLaughlin, L. W. Chang, A. Schilling, and J. M. Gregg, 2010, Appl. Phys. Lett. 96, 042904.

McQuaid, R. G. P., L.-W. Chang, and J. M. Gregg, 2010, Nano Lett. 10, 3566.

McQuaid, R. G. P., L. J. McGilly, P. Sharma, A. Gruverman, and J. M. Gregg, 2011, Nature Commun. 2, 404.

Megaw, H., and C. N. W. Darlington, 1975, Acta Cryst. Sect. A 31, 161.

Merz, W. J., 1954, Phys. Rev. 95, 690.

Meyer, B., and D. Vanderbilt, 2002, Phys. Rev. B 65, 104111.

Miller, G. D., 1998, Ph.D. thesis, Stanford, Fig. 2.8, p. 41.

Mitsui, T., and J. Furuichi, 1953, Phys. Rev. 90, 193.

Mizuuchi, K., K. Yamamoto, and M. Kato, 1997, Appl. Phys. Lett. 70, 1201 .

Morozovska, A. N., E. A. Eliseev, G. S. Svechnikov, V. Gopalan, and S. V. Kalinin, 2008, J. Appl. Phys. 103, 124110.

Morrison, F. D., P. Zubko, D. J. Jung, and J. F. Scott, 2005, Appl. Phys. Lett. 86, 152903.

Mostovoy, M., 2006, Phys. Rev. Lett. 96, 067601.

Muller, K. A., and H. Burkard, 1979, Phys. Rev. B 19, 3593.

Mulvihill, M. L., L. E. Cross, and K. Uchino, 1995, J. Am. Ceram. Soc. 78, 3345.

Myers, L.E., R.C. Eckardt, M.M. Fejer, R.L. Byer, W. R. Bosenberg, and J.W. Pierce, 1995, J. Opt. Soc. Am. B 12, 2102.

Nattermann, T., 1983, J. Phys. C 16, 4125.

Naumov, L. M. Bellaiche, S. A. Prosandeev, I. V. Ponomareva, and I. A. Kornev, 2008, U.S. Patent No. 2008-0130346.

Naumov, I., and H. Fu, 2007, Phys. Rev. Lett. 98, 077603.

Naumov, I. I., L. Bellaiche, and H. Fu, 2004, Nature (London) 432, 737.

Néel, L., 1954, Proceedings of the International Conference on Theoretical Physics, Kyoto and Tokyo 1953 (Science Council of Japan, Tokyo), p. 751.

Nelson, C. T., et al., 2011, Nano Lett. 11, 828.

Newnham, R. E., J. J. Kramer, W. A. Schulze, and L. E. Cross, 1978, J. Appl. Phys. 49, 6088.

Newton, R. R., A. J. Ahearn, and K. G. McKay, 1949, Phys. Rev. 75, 103.

Noda, K., W. Sakamoto, T. Yogo, and S. Hirano, 2000, J. Mater. Sci. Lett. 19, 69.

O’Brien, L., D. E. Read, H. T. Zeng, E. R. Lewis, D. Petit, and R. P. Cowburn, 2009, Appl. Phys. Lett. 95, 232502.

Odulov, S. G., 1982, JETP Lett. 35, 10.

Okamoto, Y., P.C. Wang, and J.F. Scott, 1985, Phys. Rev. B 32, 6787.

Ono, T., H. Miyajima, K. Shigeto, K. Mibu, N. Hosoito, and T. Shinjo, 1999a, J. Appl. Phys. 85, 6181.

Ono, T., H. Miyajima, K. Shigeto, K. Mibu, N. Hosoito, and T. Shinjo, 1999b, Science 284, 468.

Otto, T., S. Grafström, H. Chaib, and L. M. Eng, 2004, Appl. Phys. Lett. 84, 1168.

Padilla, J., W. Zhong, and D. Vanderbilt, 1996, Phys. Rev. B 53, R5969.

Palai, R., R. S. Katiyar, H. Schmid, P. Tissot, S. J. Clark, J. Robertson, S. A. T. Redfern, G. Catalan, and J.F. Scott, 2008, Phys. Rev. B 77, 014110.

Palai, R., et al., 2010, Phys. Rev. B 81, 024115.

Pan, X. Q., M. S. Hu, M. H. Yao, and Feng Duan, 1985, Phys. Status Solidi A 92, 57.

Park, S.E., and T. R. Shrout, 1997, J. Appl. Phys. 82, 1804. 
Parkin, S. S. P., M. Hayashi, and L. Thomas, 2008, Science 320, 190.

Paruch, P., T. Giamarchi, and J.-M. Triscone, 2005, Phys. Rev. Lett. 94, 197601.

Penman, Z.E., 1998, Opt. Commun. 146, 147.

Pertsev, N.A., and A. G. Zembilgotov, 1995, J. Appl. Phys. 78, 6170.

Pompe, X. Gong, Z. Suo, and J. S. Speck, 1993, J. Appl. Phys. 74, 6012.

Privratska, J., 2007, Ferroelectrics 353, 116.

Privratska, J., and V. Janovec, 1997, Ferroelectrics 204, 321.

Privratska, J., and V. Janovec, 1999, Ferroelectrics 222, 23.

Pyatakov, et al., 2011, Europhys. Lett. (in press).

Ralph, D., and M. D. Stiles, 2008, J. Magn. Magn. Mater. 320, 1190.

Randall, C. A., N. Kim, J.-P. Kucera, W. Cao, and T. R. Shrout, 1998, J. Am. Ceram. Soc. 81, 677.

Rao, Wei-Feng, and Wang U. Yu, 2007, Appl. Phys. Lett. 90, 041915.

Reid, D. T., 1997, Opt. Lett. 22, 1397.

Ren, X., Y. Wang, K. Otsuka, P. Lloveras, T. Castan, M. Porta, A. Planes, and A. Saxena, 2009, MRS Bull. 34, 838.

Reznik, L. G., et al., 1985 Ferroelectrics 64, 215.

Robels, U., and G. Arlt, 1993, J. Appl. Phys. 73, 3454.

Rodriguez, B. J., Y.H. Chu, R. Ramesh, and S. V. Kalinin, 2008, Appl. Phys. Lett. 93, 142901.

Rodriguez, B. J., X.S. Gao, L. F. Liu, W. Lee, I. I. Naumov, A. M. Bratkovsky, D. Hesse, and M. Alexe, 2009, Nano Lett. 9, 1127.

Rodriguez, B. J., S. Jesse, A. P. Baddorf, S.-H. Kim, and S. V. Kalinin, 2007a, Phys. Rev. Lett. 98, 247603.

Rodriguez, B. J., S. Jesse, A. P. Baddorf, T. Zhao, Y. H. Chu, R. Ramesh, E. A. Eliseev, A.N. Morozovska, and S. V. Kalinin, 2007b, Nanotechnology 18, 405701.

Rogan, R. C., 2003, Nature Mater. 2, 379.

Roitburd, A. L., 1976, “ Phys. Status Solidi (a) 37, 329.

Rosenman, G., P. Urenski, A. Agronin, A. Arie, and Y. Rosenwaks, 2003a, Appl. Phys. Lett. 82, 3934.

Rosenman, G., et al., 2003b, Appl. Phys. Lett. 82, 103.

Rotermund, F., 1999, Opt. Lett. 24, 1874.

Safrankova, M., J. Fousek, and S. A. Kizhaev, 1967, Czech. J. Phys. Sect. B 17, 559.

Saint-Gregoire, P., V. Janovec, E. Snoeck, C. Roucau, and Z. Zikmund, 1992, Ferroelectrics 125, 209.

Salafranca, J., R. Yu, and E. Dagotto, 2010, Phys. Rev. B 81, 245122.

Salje, E. K. H., 2010, ChemPhysChem 11, 940.

Salje, E. K. H., and H. Zhang, 2009, Phase Transit. 82, 452.

Schilling, A., T. B. Adams, R. M. Bowman, J. M. Gregg, G. Catalan, and J. F. Scott, 2006a, Phys. Rev. B 74, 024115.

Schilling, A., R. M. Bowman, G. Catalan, J.F. Scott, and J. M. Gregg, 2007, Nano Lett. 7, 3787.

Schilling, A., R. M. Bowman, J.M. Gregg, G. Catalan, and J. F. Scott, 2006b, Appl. Phys. Lett. 89, 212902.

Schilling, A., D. Byrne, G. Catalan, K. G. Webber, Y. A. Genenko, G. S. Wu, J.F. Scott, and J. M. Gregg, 2009, Nano Lett. 9, 3359.

Schilling, A., et al., 2011 (unpublished).

Schmid, H., E. Burkhardt, E. Walker, W. Brixel, M. Clin, J.-P. Rivera, J.-L. Jorda, M. François, and K. Yvon, 1988, Z. Phys. B 72, 305.

Scott, J.F., 2000, Ferroelectric Memories (Springer, New York).

Scott, J. F., 2002, in Properties of Lithium Niobate, edited by K. K. Wong (IEEE, New York).

Scott, J. F., 2006, J. Phys. Condens. Matter 18, R361.

Scott, J. F., 2007, Ferroelectrics 349, 157.
Scott, J. F., et al., 1988, J. Appl. Phys. 64, 787.

Scrymgeour, D. A., V. Gopalan, A. Itagi, A. Saxena, and P. J. Swart, 2005, Phys. Rev. B 71, 184110.

Scrymgeour, D. A., A. Sharan, V. Gopalan, K. T. Gahagan, J. L. Casson, R. Sander, J.M. Robinson, F. Muhammad, P. Chandramani, and F. Kiamilev, 2002, Appl. Phys. Lett. 81, 3140.

Seidel, J., D. Fu, S.-Y. Yang, E. Alarcòn-Lladò, J. Wu, R. Ramesh, and J. W. Ager, 2011, Phys. Rev. Lett. 107, 126805.

Seidel, J., et al., 2010, Phys. Rev. Lett. 105, 197603.

Seidel, J., et al., 2009, Nature Mater. 8, 229.

Sene, A., L. Baudry, I. A. Luk'yanchuk, and L. Lahoche, 2009, arXiv:0909.1757.

Shigenari, T., K. Abe, T. Takemoto, O. Sanaka, T. Akaike, Y. Sakai, R. Wang, and M. Itoh, 2006, Phys. Rev. B 74, 174121.

Shilo, D., G. Ravichandran, and K. Bhattacharya, 2004, Nature Mater. 3, 453.

Shinjo, T., T. Okuno, R. Hassdorf, K. Shigeto, and T. Ono, 2000, Science 289, 930.

Shur, V., et al., 2000, Appl. Phys. Lett. 76, 143.

Shur, V. Y., 2006, J. Mater. Sci. 41, 199.

Shur, V.Y., A.L. Gruverman, and E.L. Rumentsev, 1990, Ferroelectrics 111, 123.

Shuvalov, L. A., E. F. Dudnik, and S. V. Wagin, 1985, Ferroelectrics 65, 143.

Sinnamon, L. J., R. M. Bowman, and J. M. Gregg, 2001, Appl. Phys. Lett. 78, 1724.

Skumryev, V., V. Laukhin, I. Fina, X. Mart, F. Sanchez, M. Gospodinov, and J. Fontcuberta, 2011, Phys. Rev. Lett. 106, 057206.

Smith, R. T., and F. S. Welsh, 1971, J. Appl. Phys. 42, 2219.

Spanier, J. E., A. M. Kolpak, J. J. Urban, I. Grinberg, L. Ouyang, W. Soo Yun, A. M. Rappe, and H. Park, 2006, Nano Lett. 6, 735.

Speck, J. S., and W. Pompe, 1994, J. Appl. Phys. 76, 466.

Srolovitz, D. J., and J. F. Scott, 1986, Phys. Rev. B 34, 1815.

Stemmer, S., et al., 1995, Philos. Mag. A 71, 713.

Stengel, M., and N.A. Spaldin, 2006, Nature (London) 443, 679.

Stephanovich, V.A., I. A. Luk'yanchuk, and M. G. Karkut, 2005, Phys. Rev. Lett. 94, 047601

Stiles, M. D., and J. Miltat, 2006, in Spin Dynamics in Confined Magnetic Structures III: Topics in Applied Physics (Springer, Heidelberg), Vol. 101, p. 225ff.

Streiffer, S. K., J. A. Eastman, D. D. Fong, C. Thompson, A. Munkholm, M. V. Ramana Murty, O. Auciello, G. R. Bai, and G. B. Stephenson, 2002, Phys. Rev. Lett. 89, 067601.

Streiffer, S. K., C. B. Parker, A. E. Romanov, M. J. Lefevre, L. Zhao, J. S. Speck, W. Pompe, C. M. Foster, and G. R. Bai, 1998, J. Appl. Phys. 83, 2742.

Sturman, B., and V. Fridkin, 1992, The Photovoltaic and Photorefrective Effects in Noncentrosymmetric Materials (Gordon and Breach, New York).

Tagantsev, A. K., 2010, "CECAM Worskhop on the Fundamental Physics of Multiferroics, Lausanne."

Tagantsev, A. K., E. Courtens, and L. Arzel, 2001, Phys. Rev. B 64, 224107.

Tagantsev, A. K., L.E. Cross, and J. Fousek, 2010, Domains in Ferroic Crystals and Thin Films (Springer, New York).

Takahashi, R., Ø. Dahl, E. Eberg, J. K. Grepstad, and T. Tybell, 2008, J. Appl. Phys. 104, 064109.

Tarrach, G., P. Lagos, R. Hermans, F. Schlaphof, C. Loppacher, and L. M. Eng, 2001, Appl. Phys. Lett. 79, 3152.

Thiele, A. A., 1970, J. Appl. Phys. 41, 1139.

Thomas, L., R. Moriya, C. Rettner, and S.S.P. Parkin, 2010, Science 330, 1810. 
Tian, L., 2006, Ph.D. thesis Pennsylvania State University.

Tiihonen, M., V. Pasiskevicius, and F. Laurell, 2006, Opt. Express 14, 8728.

Toledano, J.-C., 1974, Annales des Telecommun. 29, 249.

Toledano, J.-C., and P. Toledano, 1987, The Landau Theory of Phase Transitions (World Scientific, Singapore).

Tybell, T., P. Paruch, T. Giamarchi, and J.-M. Triscone, 2002, Phys. Rev. Lett. 89, 097601.

Uesu, Y., R. Nakai, J.-M. Kiat, C. Me Noret, M. Itoh, and T. Kyomen, 2004, J. Phys. Soc. Jpn. 73, 1139.

Urban, K., et al., 2008, Eds., Advances in Imaging and Electron Physics (Elsevier, New York), Vol. 153, p. 439.

Van Aken, B. B., T. T. M. Palstra, A. Filippetti, and N. A. Spaldin, 2004, Nature Mater. 3, 164.

Viret, M., Y. Samson, P. Warin, A. Marty, F. Ott, E. Søndergård, O. Klein, and C. Fermon, 2000, Phys. Rev. Lett. 85, 3962.

Vlooswijk, A. H. G., G. Catalan, and Beatriz Noheda, 2010, Appl. Phys. Lett. 97, 046101.

Vlooswijk, A.H.G., B. Noheda, G. Catalan, A. Janssens, B. Barcones, G. Rijnders, D. H. A. Blank, S. Venkatesan, B. Kooi, and J. T. M. de Hosson, 2007, Appl. Phys. Lett. 91, 112901.

Wada, S., K. Yako, K. Yokoo, H. Kakemoto, and T. Tsurumi, 2006, Ferroelectrics, 334, 17.

Wang, S., 1998, Opt. Lett. 23, 1883.

Warner, A. W., G. A. Coquin, and F. L. Frank, 1969, J. Appl. Phys. 40, 4353.

Watanabe, Y., 2007, Ferroelectrics 349, 190.

Wiessner, A., J. Kirschner, G. Schäfer, and Th. Berghaus, 1997, Rev. Sci. Instrum. 68, 3790.
Wittborn, J., C. Canalias, K. V. Rao, R. Clemens, H. Karlsson, and F. Laurell, 2002, Appl. Phys. Lett. 80, 1622.

Xiao, Y., V. B. Shenoy, K. Bhattacharya, Depletion Layers, and Domain Walls, 2005, Phys. Rev. Lett. 95, 247603.

Xu, G., Z. Zhong, Y. Bing, Z.-G. Ye, and G. Shirane, 2006, Nature Mater. 5, 134.

Yamada, M., N. Nada, M. Saitoh, and K. Watanabe, 1993, Appl. Phys. Lett. 62, 435.

Yang, B., N. J. Park, B. I. Seo, Y.H. Oh, S. J. Kim, S. K. Hong, S. S. Lee, and Y. J. Park, 2005, Appl. Phys. Lett. 87, 062902

Yang, C.-H., et al., 2009, Nature Mater. 8, 485.

Yang, S.-Y., et al., 2010, Nature Nanotech. 5, 143.

Yang, T. J., 1999, Phys. Rev. Lett. 82, 4106.

Zeng, H. R., et al., 2008, Phys. Status Solidi Rapid Res. Lett. 2, 123.

Zeng, H. T., et al., 2010, Appl. Phys. Lett. 96, 262510.

Zhang, Q. M., H. Wang, N. Kim, and L.E. Cross, 1994, J. Appl. Phys. 75, 454.

Zhao, T., et al., 2006, Nature Mater. 5, 823.

Zhirnov, V. A., 1959, Sov. Phys. JETP 35, 822.

Zubko, P., G. Catalan, A. Buckley, P. R. L. Welche, and J. F. Scott, 2007, Phys. Rev. Lett. 99, 167601.

Zubko, P., S. Gariglio, M. Gabay, P. Ghosez, and J.-M. Triscone, 2011, Annu. Rev. Condens. Matter Phys. 2, 141.

Zubko, P., N. Stucki, C. Lichtensteiger, and J.-M. Triscone, 2010, Phys. Rev. Lett. 104, 187601.

Zubko, P., and J.-M. Triscone, 2009, Nature (London) 460, 45.

Zubko, Pavel, 2008, Ph.D. thesis, University of Cambridge. 